\author{
Monograph \\ urn:1sid:zoobank.org:pub:90509851-4A60-4A51-8D39-64457010B6EE
}

\title{
Umbyquyra gen. nov., a new tarantula spider genus from the Neotropical region (Araneae, Mygalomorphae, Theraphosidae), with a description of eight new species
}

\author{
Fabio de F. GARGIULO ${ }^{1}$, Antonio D. BRESCOVIT ${ }^{2, *} \&$ Sylvia M. LUCAS ${ }^{3}$ \\ ${ }^{1,2,3}$ Laboratório Especial de Coleções Zoológicas, Instituto Butantan, \\ Av. Vital Brasil, 1500, São Paulo, Brazil. \\ *Corresponding author: antonio.brescovit@butantan.gov.br \\ ${ }^{1}$ Email: fabiofgargiulo@gmail.com \\ ${ }_{3}^{3}$ Email: sylvia.lucas@butantan.gov.br \\ ${ }^{1}$ urn:1sid:zoobank.org:author:7DDF3C5C-ABDD-4B85-9B8E-0B4EF8B689EC \\ ${ }^{2}$ urn:1sid:zoobank.org:author:D5B81D79-AFAE-47B1-8A6E-DAB448A24BCC \\ ${ }^{3}$ urn:1sid:zoobank.org:author:62B39697-00B1-482F-8C4B-946BCE83484B
}

\begin{abstract}
Umbyquyra gen. nov., a new Theraphosinae genus with stridulatory bristles on the palpal trocanther of pedipalp trochanter and first leg, is proposed. The genus differs from the other genera with stridulatory bristles on the same segments, Acanthoscurria Ausserer, 1871, Cyrtopholis Simon, 1892, Longilyra Gabriel, 2014 and Nesipelma Schmidt \& Kovarik, 1996, by having a palpal bulb with a very short and acuminate embolus and four short keels; separated tibial apophysis; and female spermathecae resembling those of Cyrtopholis, with two seminal receptacles with elongated ducts emerging from a common area. Cyrtopholis palmarum Schiapelli \& Gerschman, 1945 and C. schmidti Rudloff, 1996 from Brazil and Acanthoscurria acuminata Schmidt \& Tesmoingt in Schmidt, 2005 from Bolivia are transferred to the new genus. The female of Umbyquyra palmarum (Schiapelli \& Gerschman, 1945) gen. et comb. nov. and the male of $U$. schmidti (Rudloff, 1996) gen. et comb. nov. are described for the first time. Cyrtopholis zorodes Mello-Leitão, 1923 is considered a junior synonym of Acanthoscurria gomesiana Mello-Leitão, 1923 and Cyrtopholis meridionalis (Keyserling, 1891) is considered a nomen dubium. Eight new species from Brazil are described: Umbyquyra paranaiba gen. et sp. nov., U. cuiaba gen. et sp. nov., U. araguaia gen. et sp. nov., U. sapezal gen. et sp. nov., U. belterra gen. et sp. nov., $U$. caxiuana gen. et sp. nov., U. tucurui gen. et sp. nov. and U. tapajos gen. et sp. nov. Data and maps on the geographic distribution are provided.
\end{abstract}

Keywords. Theraphosinae, taxonomy, stridulatory bristles, new genus.

Gargiulo F. de F., Brescovit A.D \& Lucas S.M. 2018. Umbyquyra gen. nov., a new tarantula spider genus from the Neotropical region (Araneae, Mygalomorphae, Theraphosidae), with a description of eight new species. European Journal of Taxonomy 457: 1-50. https://doi.org/10.5852/ejt.2018.457 


\section{Introduction}

The family Theraphosidae Thorell, 1869 currently has eleven subfamilies (Guadanucci 2014), four of them with species described from tropical and subtropical regions: Aviculariinae, Theraphosinae, Schismatothelinae and Ischnocolinae. The latter was established by Simon (1903) based on the presence of a divided tarsal scopula on the posterior legs. He included several genera in this subfamily, among them Cyrtopholis Simon, 1892, which he cited as being the only genus with a type IV stridulatory apparatus (stridulatory bristles on palp trochanter and leg I). Raven (1985) transferred Cyrtopholis to Theraphosinae based on the large subtegulum, presence of keels on palpal bulb and retrolateral femur IV with scopula.

At present, the following four Theraphosinae genera are described as having stridulatory bristles on the palpal trochanter and on the first legs: Acanthoscurria Ausserer, 1871, which includes 25 species distributed in South America and one from Lesser Antilles (Fig. 1); Cyrtopholis Simon, 1892, with 23 species from the Caribbean region and four species from Brazil (Fig. 2), C. meridionalis (Keyserling, 1891), C. palmarum Schiapelli \& Gerschman, 1945, C. zorodes Mello-Leitão, 1923 and C. schmidti Rudloff, 1996 (World Spider Catalog 2016); Nesipelma Schmidt \& Kovarik, 1996, a monotypic genus with the type species $N$. insulare Schmidt \& Kovarik, 1996 from the Lesser Antilles (Fig. 4); and Longilyra Gabriel, 2014, also monotypic with the type species L. johnlonghorni Gabriel, 2014 from E1 Salvador (Fig. 3).

While revising the four Brazilian species of Cyrtopholis, we found that none of them could be included in this genus. Studies of the type species, Cyrtopholis cursor (Ausserer, 1875), revealed differences in the spermathecae when compared to the Brazilian species. Also, a very different morphology of the male palp bulb was observed when compared to the Caribbean species of Cyrtopholis.

The study of type material and large samples of specimens from several Brazilian collections allowed us to establish the new genus Umbyquyra gen. nov., which includes eleven species: two transferred from Cyrtopholis, another from Acanthoscurria and eight new species. The female of Umbyquyra palmarum (Schiapelli \& Gerschman, 1945) gen. et comb. nov. and the male of U. schmidti (Rudloff, 1996) gen. et comb. nov. are herein described for the first time. Data on the geographical distribution are provided for all species.

\section{Material and methods}

The examined specimens are deposited in the following taxonomic collections (curators indicated between parentheses):

\begin{tabular}{|c|c|c|}
\hline T/UNAMA & $=$ & $\begin{array}{l}\text { Faculdades Integradas do Tapajós/Faculdade da Amazônia, Santarém, Pará, Brazil } \\
\text { (H. Chalkidis) }\end{array}$ \\
\hline IBSP & $=$ & Instituto Butantan, São Paulo, Brazil (A.D. Brescovit) \\
\hline MACN & $=$ & $\begin{array}{l}\text { Museo Argentino de Ciencias Naturales "Bernadino Rivadavia", Buenos Aires, } \\
\text { Argentina (C. Scioscia and M.J. Ramírez) }\end{array}$ \\
\hline MNHNSD & $=$ & $\begin{array}{l}\text { Museo Nacional de Historia Natural Eugeneo Marcano, Santo Domingo, Dominican } \\
\text { Republic (G. de Los Santos) }\end{array}$ \\
\hline MPEG & $=$ & Museu Paraense Emilio Goeldi, Belém, Pará, Brazil (A.B. Bonaldo) \\
\hline MZSP & $=$ & $\begin{array}{l}\text { Museu de Zoologia da Universidade de São Paulo, São Paulo, Brazil (R. Pinto da } \\
\text { Rocha) }\end{array}$ \\
\hline SMF & $=$ & Senckenberg Museum, Frankfurt, Germany (P. Jäger) \\
\hline UFMT & $=$ & Universidade Federal de Mato Grosso, Cuiabá, Brazil (A. Chagas) \\
\hline ZMB & $=$ & Zoologisches Museum Berlin, Berlin, Germany (J. Dunlop) \\
\hline
\end{tabular}


Male palpal bulb terminology follows Bertani (2000) with some modifications in the abbreviations. Spine number and disposition follow Petrunkevitch (1925), with modifications proposed by Bertani (2001). All measurements are in millimeters and were taken using a Leica DFC425 stereo microscope with camera lucida. Length of leg segments were measured between joints, in dorsal view. Total body length excludes chelicerae and spinnerets. Extended focal range images were composed with Leica Application
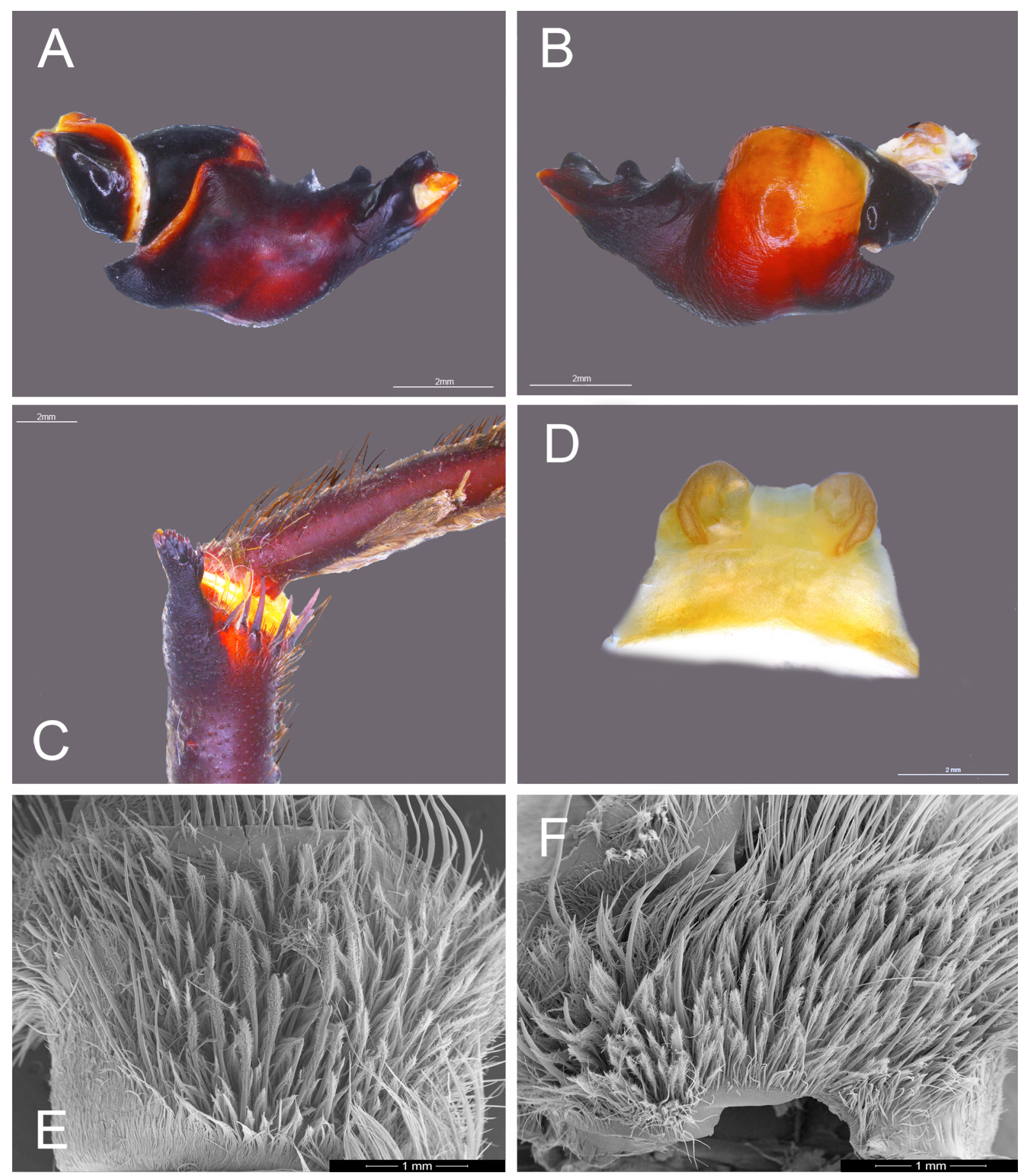

Fig. 1. Acanthoscurria geniculata Ausserer, 1871, đ̊ and $\subsetneq$, Santarém, Pará, Brazil (IBSP 151260). A-B. Palpal bulb. A. Retrolateral. B. Prolateral. C. Tibial apophysis, retrolateral. D. Spermathecae, dorsal. E. Male palp, trochanter, stridulatory organ. F. Trochanter I, stridulatory organ. 
Suite version 2.5.0 (available from http://www.heliconsoft.com/heliconsoft-products/helicon-focus/). All photos of scanning electronic microscopy (SEM) were taken under high vacuum with a FEI Quanta 250 SEM at the Laboratório de Biologia Celular of the Instituto Butantan. Spermathecae were dissected and immersed in enzyme (Ultrazyme ${ }^{\circledR}$ ) for 72 hours for soft tissue digestion to allow observation of internal structures.
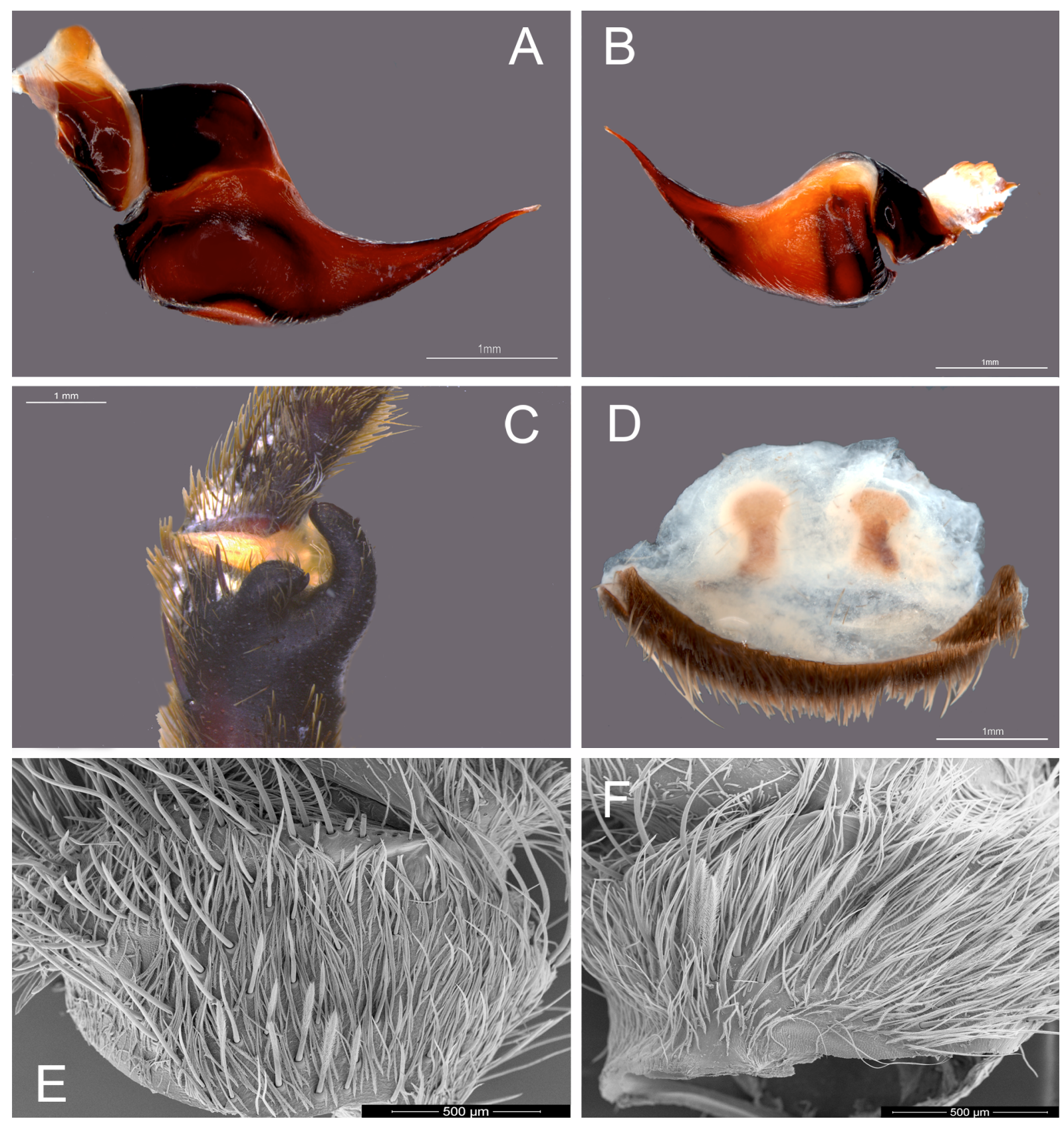

Fig. 2. Cyrtopholis sp., $\hat{O}$ (MNHNSD 09.1463) and $q$ (MNHSD 1454), Parque Nacional Valle Nuevo, Dominican Republic. A-B. Palpal bulb. A. Retrolateral. B. Prolateral. C. Tibial apophysis, retrolateral. D. Spermathecae, female, dorsal. E. Male palp, trochanter, stridulatory organ. F. Trochanter I, stridulatory organ. 
Geographic coordinates were obtained using a GPS, for specimens collected by us, or through information on the Museum original labels. Localities from museum samples without coordinates were georeferenced using Google Earth ${ }^{\circledR}$.

\section{Morphological abbreviations}

$\begin{array}{ll}\mathrm{A} & =\text { apical keel } \\ \mathrm{ALE} & =\text { anterior lateral eyes } \\ \mathrm{AME} & =\text { anterior median eyes } \\ \mathrm{ap} & =\text { apical } \\ \mathrm{app} & =\text { apical prolateral } \\ \mathrm{apr} & =\text { apical retrolateral } \\ \mathrm{BA} & =\text { basal area } \\ \mathrm{d} & =\text { dorsal } \\ \mathrm{p} & =\text { prolateral } \\ \mathrm{PAc} & =\text { prolateral accessory keel } \\ \mathrm{PB} & =\text { prolateral branch } \\ \mathrm{PI} & =\text { prolateral inferior keel } \\ \mathrm{PLE} & =\text { posterior lateral eyes }\end{array}$
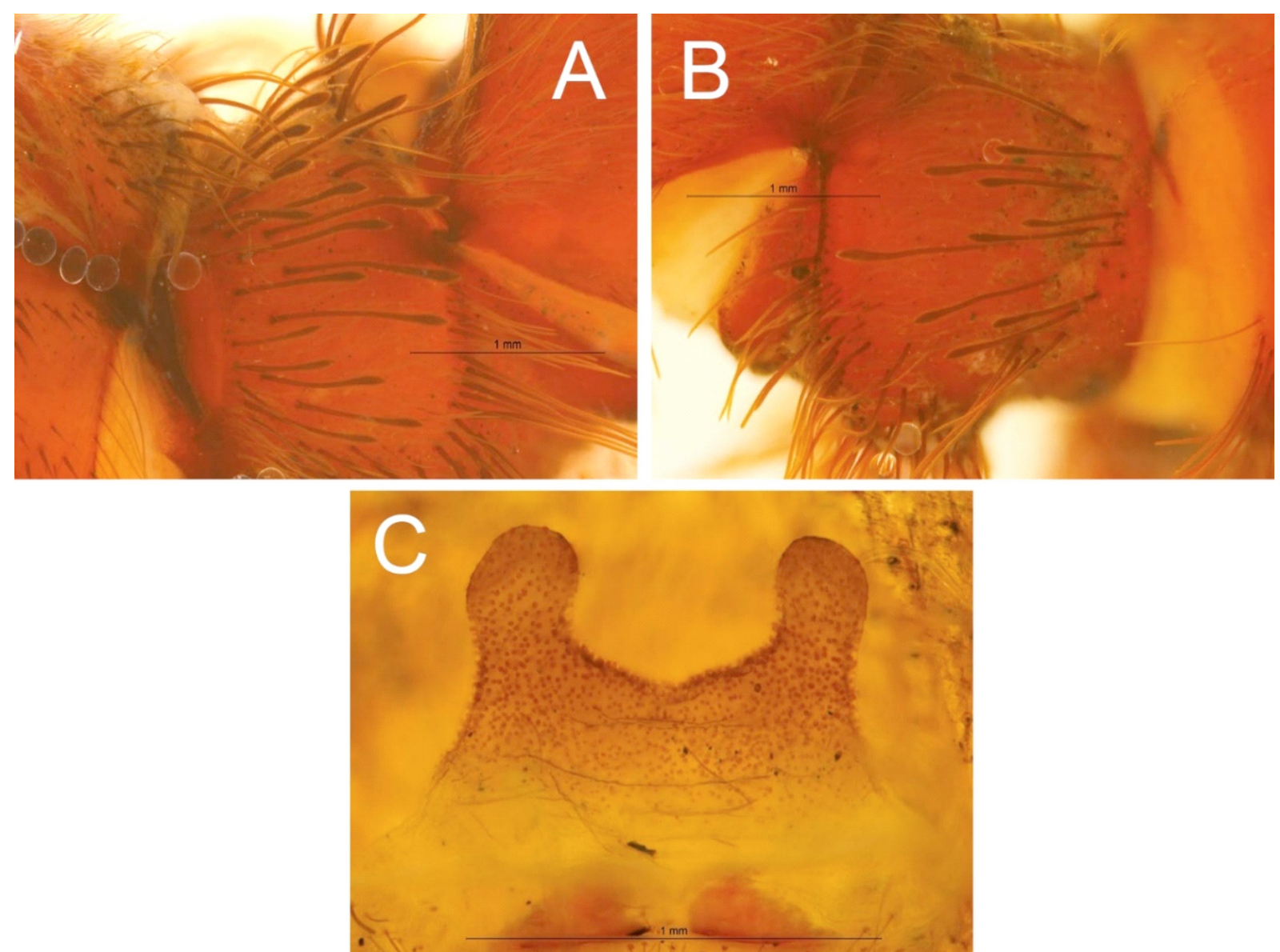

Fig. 3. Longilyra johnlonghorni Gabriel, 2014,, , Santa Ana, Laguna de Las Ninfas, El Salvador (SMF $8582 / 2$ ). A. Palp, trochanter, stridulatory organ. B. Trochanter I, stridulatory organ. C. Spermathecae, dorsal. From Gabriel (2014: figs 1, 2, 5). 

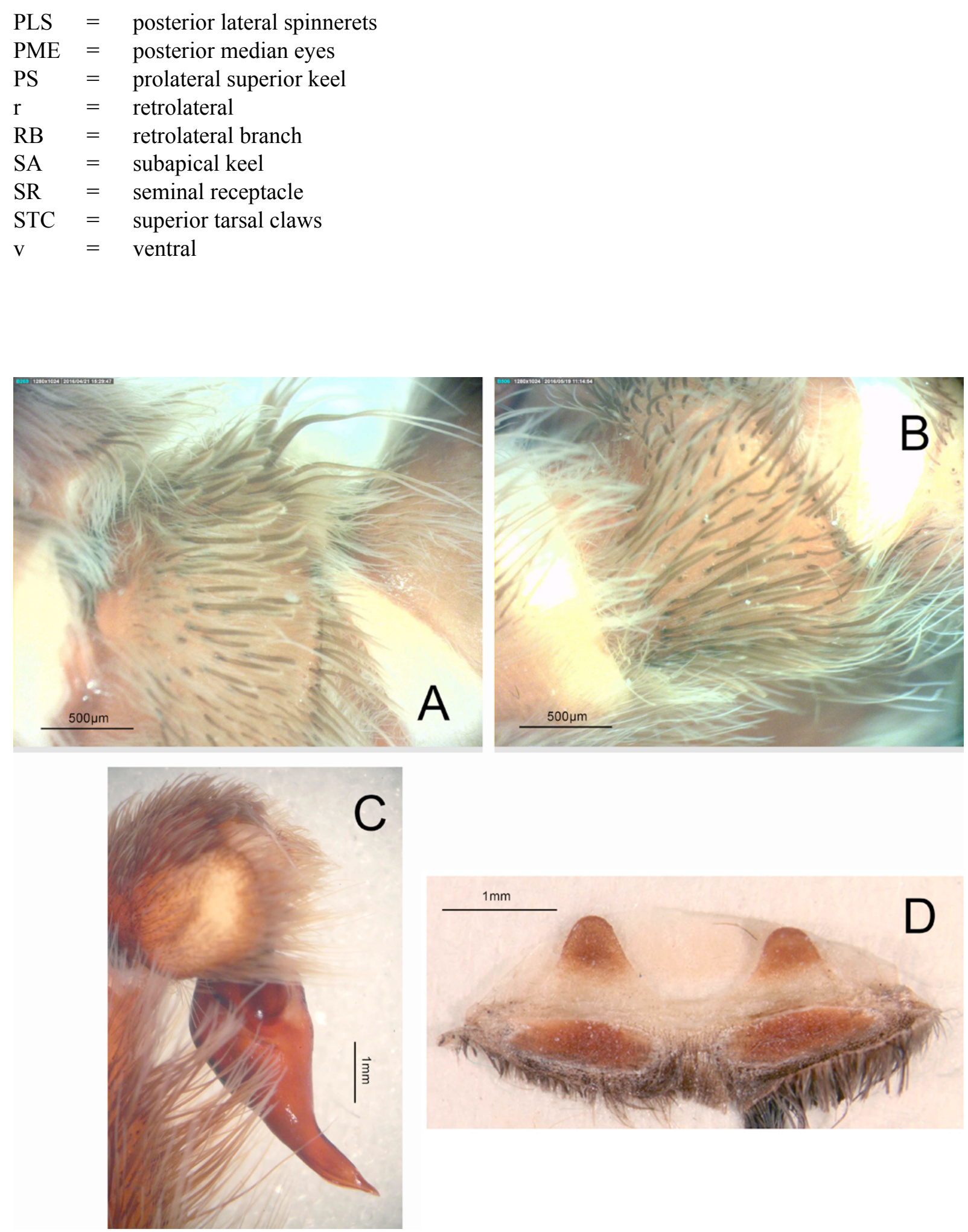

Fig. 4. Nesipelma insulare Schmidt \& Kovarik, 1996, ô (SMF 38557-84) and $q$ (SMF 39188-84) from the Lesser Antilles, Nevi Island. A-C. Male palp. A. Trochanter, stridulatory organ. B. Trochanter I, stridulatory organ. C. Retrolateral. D. Spermathecae, female, dorsal (Photos by P. Jäger and C. Perafán). 


\section{Comparative material}

Acanthoscurria geniculata Ausserer, 1871

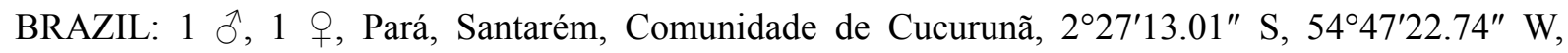
25 Dec. 2009, A.P. Silva leg. (IBSP 151260) (Fig. 1).

\section{Cyrtopholis sp.}

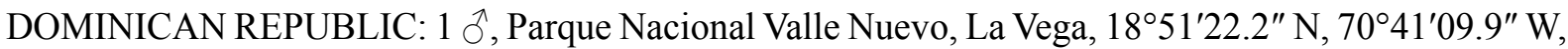
10 May 2001, D. Veloz leg. (MNHNSD 09.1463); 1 , same data, 29 Aug. 2009, G. de Los Santos leg. (MNHNSD 09.1454) (Fig. 2).

\section{Longilyra johnlonghorni Gabriel, 2014}

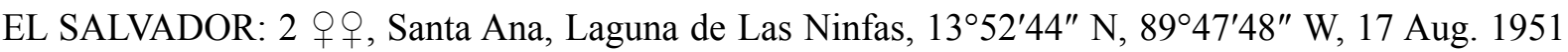
(SMF 8582/2A) (Fig. 3).

Nesipelma insulare Schmidt \& Kovarik, 1996

LESSER ANTILLES: 1 ○े, holotype, Nevi Island, 17 Apr. 1993, Bezder leg. (SMF 38557-84); 1 q, same data as for holotype (SMF 39188-84) (Fig. 4).

\section{Results}

Class Arachnida Cuvier, 1812

Order Araneae Clerck, 1757

Family Theraphosidae Thorell, 1869

Subfamily Theraphosinae Thorell, 1869

Umbyquyra gen. nov.

urn:Isid:zoobank.org:act:4AE10C23-FC0A-4379-B255-22DE2FAF022C

\section{Type species}

Umbyquyra paranaiba gen. et sp. nov.

\section{Diagnosis}

Umbyquyra gen. nov. presents stridulatory bristles on the palpal trocanther and first leg as the genera Acanthoscurria, Cyrtopholis, Nesipelma and Longilyra. It differs from these genera by a projected and well marked cephalic region (Fig. 5) and mainly by the aspect of the genitalia: male palpal bulb compact and globose, tegulum short, embolus, palpal bulb with a short embolus presenting four keels, tibial apophysis with two branches not originating from a common base, and metatarsus I, when flexed, touches the retrolateral branch laterally (Figs 7C, 8D); female spermathecae with SR connected to a sclerotized basal plate with conspicuous ducts (Fig. 7D).

\section{Etymology}

Umbyquyra gen. nov. originates from the Tupi Indian language and means 'pointed bird beak', relative to the aspect of the short embolus of the palpal bulb. The gender is neuter. 
A

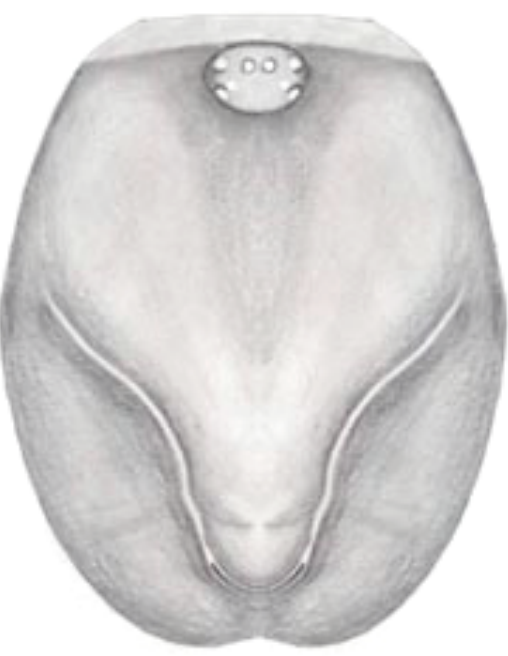

C

C 5009

䁬

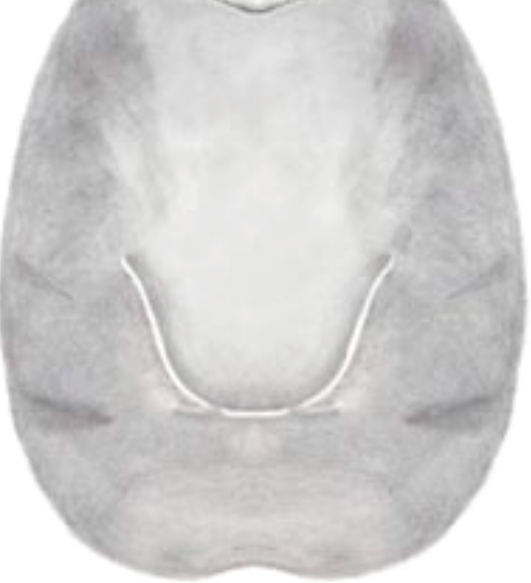

E

E

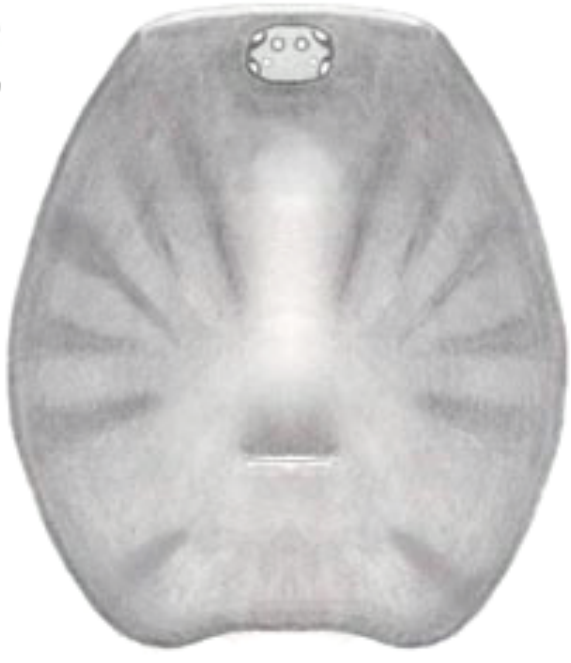

B

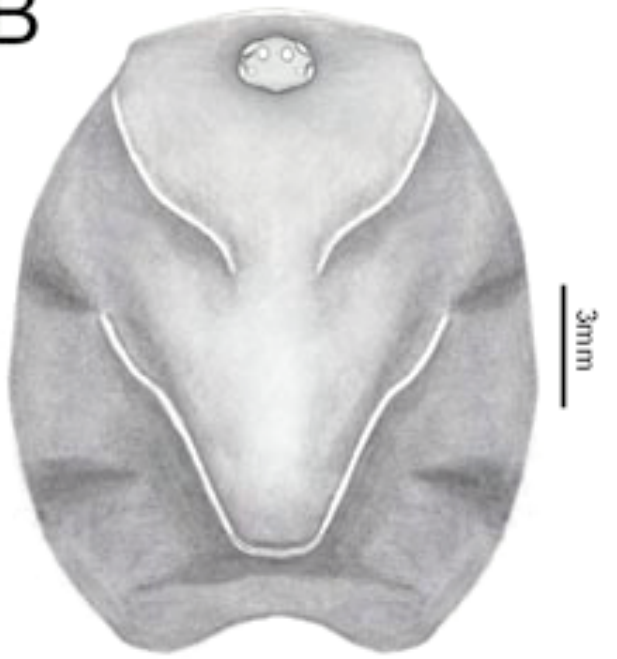

D
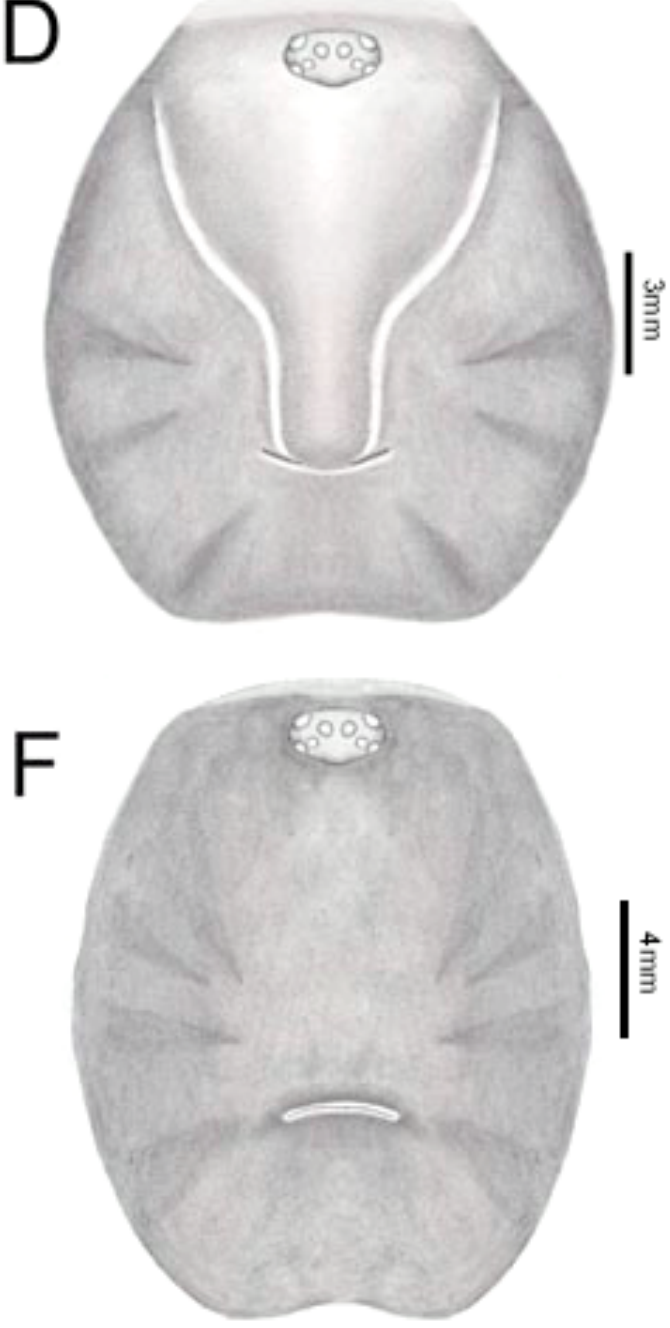

Fig. 5. Fovea, carapace. A. Umbyquyra schimidti gen. et sp. nov. B. U. sapezal gen. et sp. nov. C. U. caxiuana gen. et sp. nov. D. U. cuiaba gen. et sp. nov. E. U. paranaiba gen. et sp. nov. F. U. belterra gen. et sp. nov. 

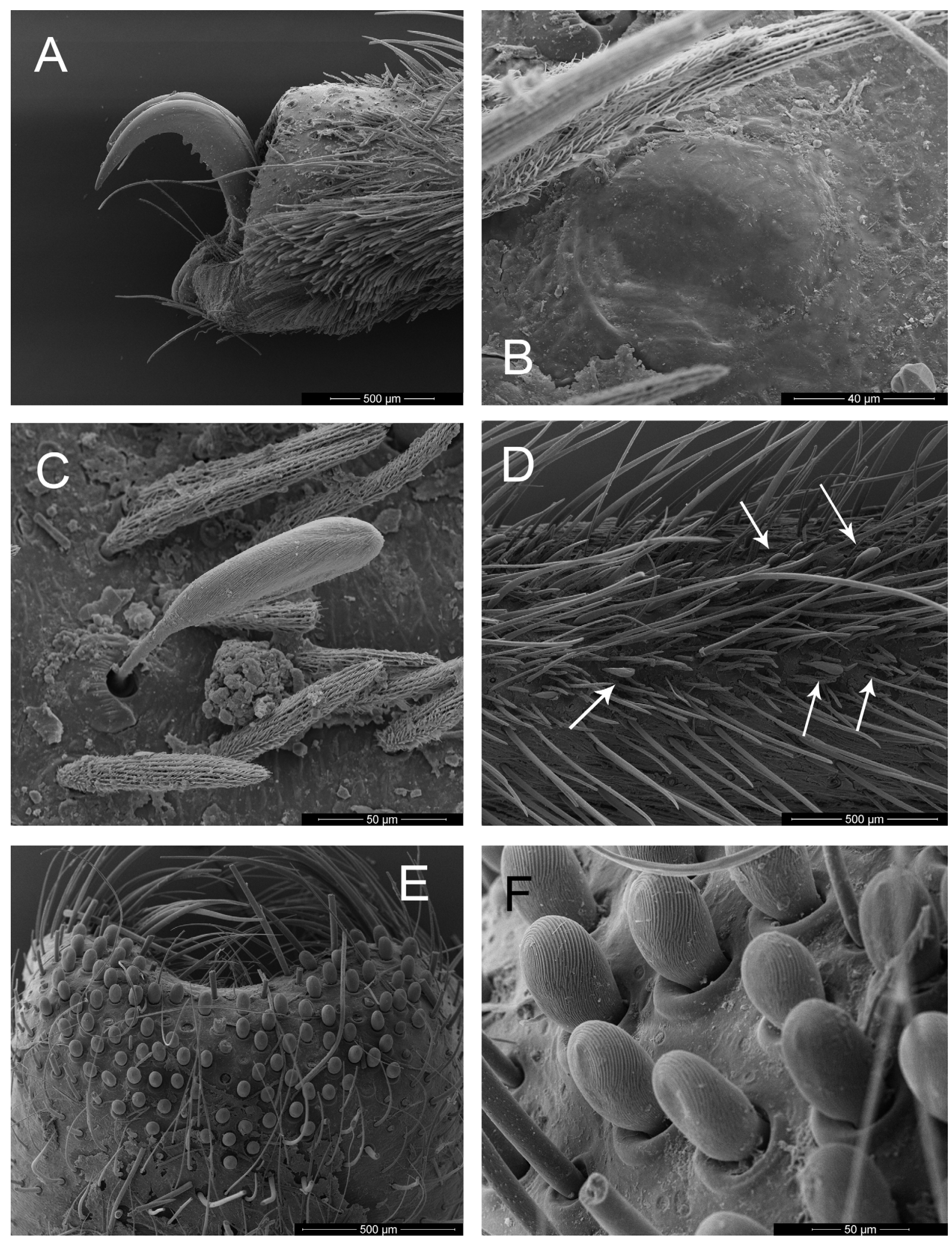

Fig. 6. Umbyquyra tucurui gen. et sp. nov., đ̂, from Tucuruí, Pará, Brazil (IBSP 104716). A. Tarsal claws, leg I, prolateral view. B. Tarsal organ, dorsal. C. Trichobothrium, tarsi I, lateral. D. Row of trichobotria, tarsi I, dorsal (arrows indicate the trichobothria). E. Labium, ventral. F. Cuspules of labium, detail. 


\section{Description}

Total length: males $18-40$, females $20-44$. General color is dark brown with legs lighter brown. The dorsal side of abdomen covered with long reddish or golden hairs with a dark patch (Fig 16F), ventral side of abdomen dark brown, legs with long yellowish or reddish hairs (Fig. 16F). Carapace longer than wide. Cephalic region with a projection slightly raised defined by the thoracic grooves. Fovea procurve (Fig. 5A-B, D), straight (Fig. 5C, E) or recurve (Fig. 5F), some species with projection over the fovea (Fig. 15E). Eye tubercle slightly raised, wider than long. Eight eyes, ringed with black. Anterior row of eyes procurved, posterior row slightly recurved or straight (Fig. 16E). Labium as wide as long, quadrate, with 130 cuspules (Fig. 6E). Endites with distinct anterior lobe, with 90-230 cuspules on internal basal angle. Sternum oval with six sigilla. Tarsal scopulae dense and integral, only tarsus IV with some bristles in longitudinal line. STC with 4-5 small teeth (Fig. 6A). Tarsi I-IV with two rows of clavate trichobotria (Fig. 6C-D). Scopulae on all tarsi and metatarsi I-II integral and only apical on metatarsi III-IV. Stridulatory bristles on palpal trochanter with 13-25 bristles and 30-38 on trocanther of leg I, sometimes with very long bristles similar as the described for Longilyra, occupying all the length of trochanter in other difficult to observe as in Umbyquyra acuminata (Schmidt \& Tesmoingt in Schmidt, 2005) gen. et comb. nov. (Fig. 14E-F). Umbyquyra palmarum gen. et comb. nov. (Fig. 12E-F), U. tapajos gen. et sp. nov. (Fig. 24E-F), U. tucurui gen. et sp. nov. (Fig. 26E-F), U. caxiuana gen. et sp. nov. (Fig. 28E-F) have bristles long and very large, varying in size and number. PLS threesegmented with digitiform apical segment. Male palpal bulb with oval tegulum, short embolus, four or five keels resembles Acanthoscurria, but differs by distribution of keels. Tibia I with two branches, not originating from common base, retrolateral and prolateral branch with strong spines; palpal bulb with oval tegulum (Figs 7A-C, 8A-D), in general with four keels (PS, PI, SA, A), but two species have a prolateral accessory keel (PAc). Umbyquyra palmarum gen. et comb. nov. have four keels. Female genitalia: spermatheca with heavy sclerotized basal area (Fig. 7D), in U. cuiaba gen. et sp. nov. basal area reduced, SR with short (Fig. 11D) or elongated (Figs 7D, 15D) ducts emerging or not from the basal area. Urticating hairs of type I and III present in both sexes.

\section{Species included}

Umbyquyra paranaiba gen. et sp. nov., U. cuiaba gen. et sp. nov., U. araguaia gen. et sp. nov., U. sapezal gen. et sp. nov., U. belterra gen. et sp. nov., U. caxiuana gen. et sp. nov., U. tucurui gen. et sp. nov., $U$. tapajos gen. et sp. nov., U. palmarum gen. et comb. nov., U. acuminata gen. et comb. nov. and U. schmidti gen. et comb. nov.

\section{Distribution}

Species of Umbyquyra gen. nov. are registered in the Brazilian states of Acre, Pará, Mato Grosso, Tocantins, Goiás, Mato Grosso do Sul, São Paulo and Bolivia, Province Sara (Figs 29-31).

Umbyquyra paranaiba gen. et sp. nov. urn:1sid:zoobank.org:act:A251D3E2-A7B2-4445-9FC1-4DA4D9F76A4F

Figs 5E, 7-8, 18G-H, 29

Cyrtopholis palmarum - Bertani 2000: 30-31 (misidentification).

\section{Diagnosis}

Males of $U$. paranaiba gen. et sp. nov. differ from those of the other species by the short and straight fovea (Fig. 5E), a palpal bulb with a short embolus, the prolateral inferior (PI) and apical keel (A) parallel near the rear distancing from each other in the middle region, becoming more evident and narrowing again at the rear (Figs 7A, 8B) and tibial apophysis with a very short prolateral branch with 
two strong distinct spines (Figs 7C, 8D). Females resemble those of U. palmarum gen. et comb. nov. by an SR with short ducts, but differ by a rectangular and broader sclerotized basal area (Fig. 7D).

\section{Note}

Bertani (2000: 30-31) identify a male as C. palmarum (IBSP 4730). The specimen was examined by us and identified as $U$. paranaiba gen. et sp. nov. and we detected that the number of keels is four (PS, PI, A and SA, Fig. 8) and not three.

\section{Etymology}

The species epithet is a noun in apposition taken from the type locality.

\section{Type material}

\section{Holotype}

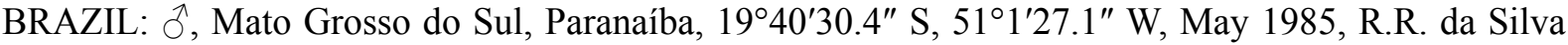
leg. (IBSP 107411).
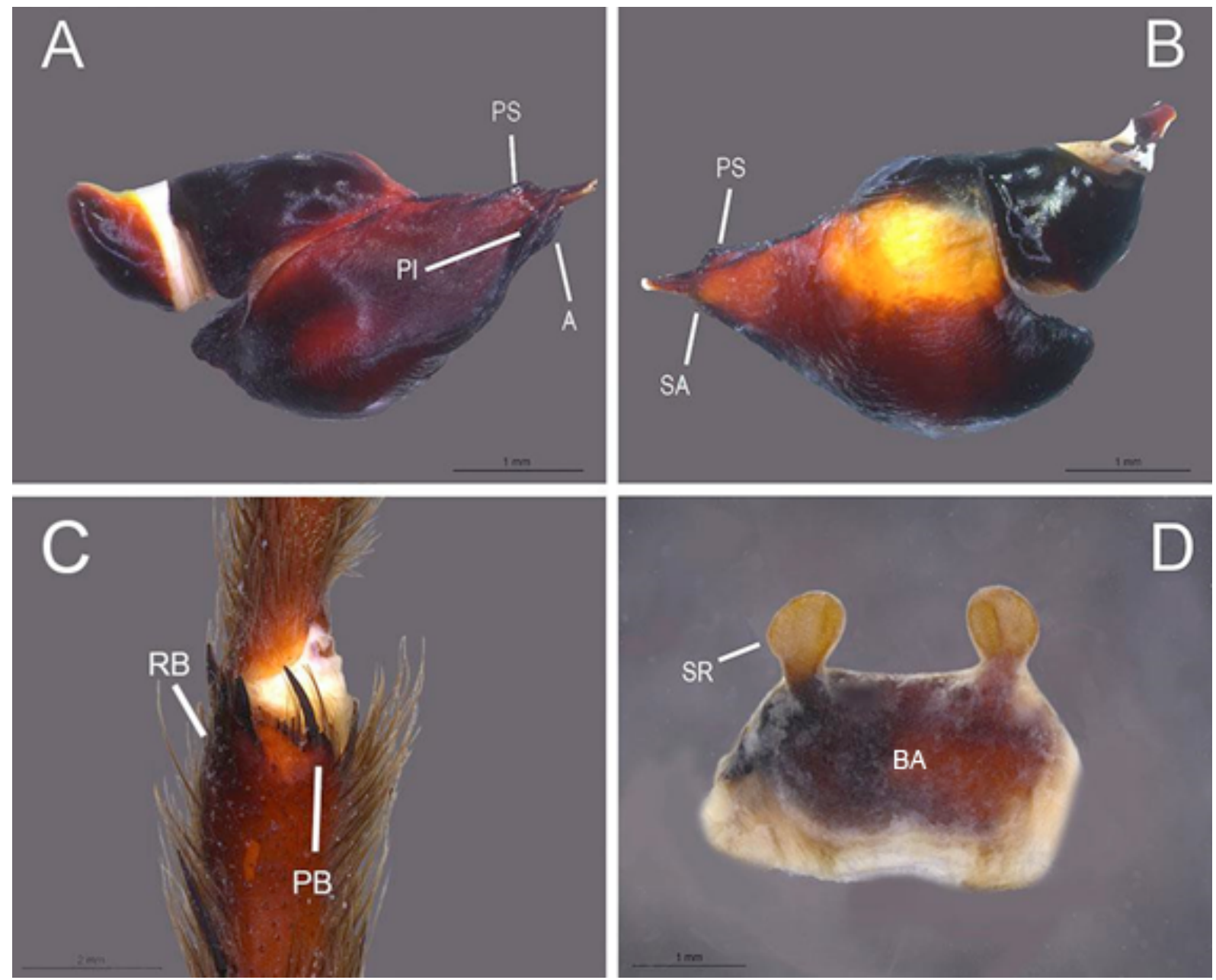

Fig. 7. Umbyquyra paranaiba gen. et sp. nov. A-C. Holotype, ô (IBSP 107411). A. Palp, prolateral. B. Retrolateral. C. Tibial apophysis, ventral. D. Paratype, $q$ (IBSP 111695), spermathecae, dorsal. Abbreviations: see Material and methods. 
Paratypes

BRAZIL: Acre: $1{ }^{\Uparrow}$, Rio Branco, 958'25.7" S, 6749'29.7" W, 13 Oct. 1952, C.A. Souza leg. (IBSP

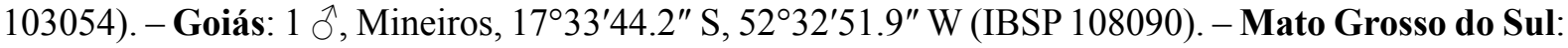

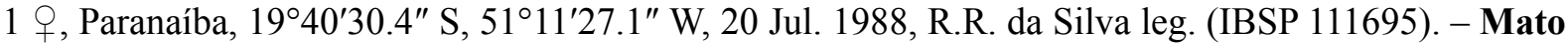

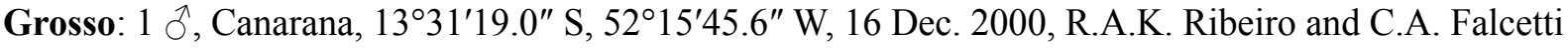
leg. (IBSP 110469). - São Paulo: 2 ô $^{\text {ò }}$, Morro Agudo, 2043'48.6" S, 4803'15.0" W, Oct. 1987, J.H.B. Medeiros leg. (IBSP 107421).

\section{Other material examined}

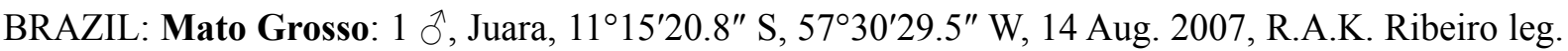
(UFMT 0964); 1 đ’, Cuiabá, $15^{\circ} 36^{\prime} 44.9^{\prime \prime} \mathrm{S}, 56^{\circ} 04^{\prime} 05.8^{\prime \prime} \mathrm{W}, 2002$ (UFMT 0965); 1 ô, same collection data

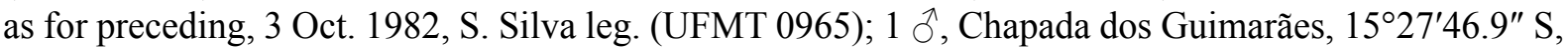

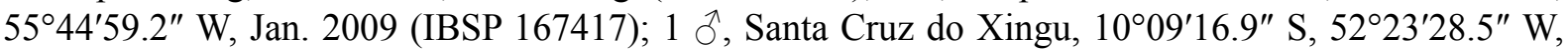

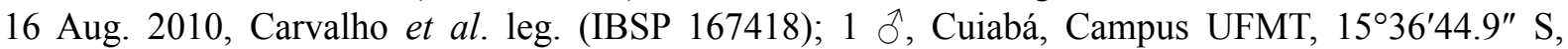

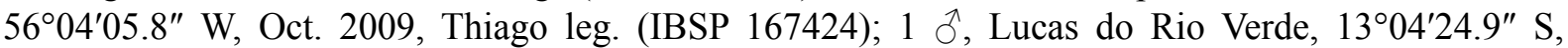

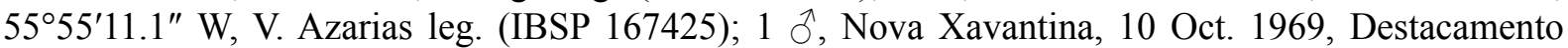

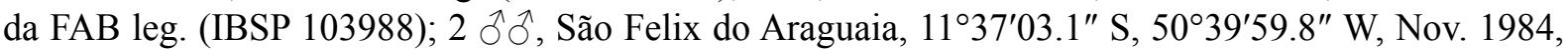

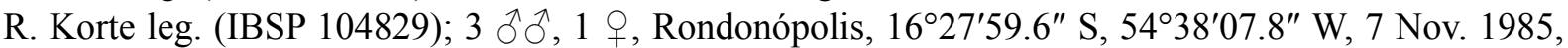
Secretaria Municipal de Saúde leg. (IBSP 104896); $1 \delta^{\lambda}$, Parque Nacional do Xingu, $11^{\circ} 32^{\prime} 45^{\prime \prime}$ S,

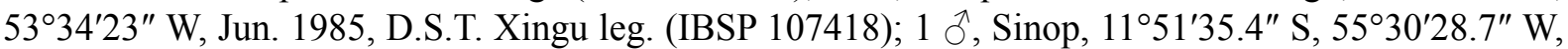

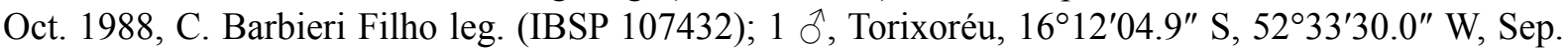

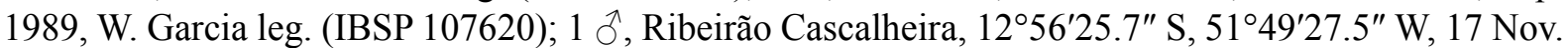
2011 (IBSP 166975); $1 \hat{\jmath}$, same collection data as for preceding (IBSP 166976); $1 \hat{\jmath}$, same collection data as for preceding (IBSP 166977); $1 \hat{\delta}$, same collection data as for preceding (IBSP 166978). - Mato

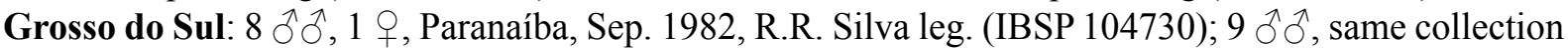
data as for preceding, Oct. 1982 (IBSP 104733A); 6 ఓぇ, same collection data as for preceding, Oct.

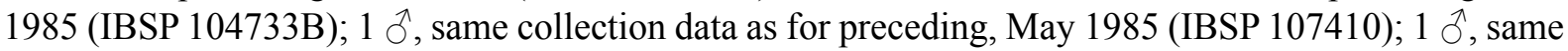
collection data as for preceding, Jun. 1985 (IBSP 107419); 1 ऽ, same collection data as for preceding, Jun. 1985 (IBSP 107420); 1 $\hat{\text {, }}$, same collection data as for preceding, Aug. 1983 (IBSP 107621);

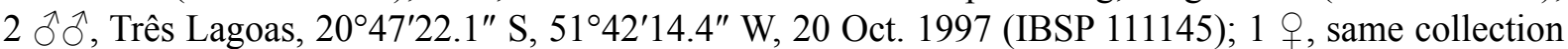
data as for preceding, Sep. 1997, C. Gerbi leg. (IBSP 111145); 1 §, Campo Grande, 20²8'06.8" S, $54^{\circ} 37^{\prime} 07.1^{\prime \prime} \mathrm{W}, 20$ Oct. 1985, Vera and Cesar leg. (IBSP 111693); 1 $\partial^{\circ}$, Chapadão do Sul, Fazenda São Roque, $18^{\circ} 47^{\prime} 42.5^{\prime \prime} \mathrm{S}, 52^{\circ} 37^{\prime} 05.5^{\prime \prime} \mathrm{W}$, Oct. 2007, C. Pritsch leg. (IBSP 115381).

\section{Natural History}

Very aggressive when disturbed; specimens raise the abdomen resembling the defensive display of the species of Avicularia and of some spiders of Nemesiidae, or raise pedipalps and legs I resembling species of Acanthoscurria (Fig. 18D, H; Gonzalez-Filho et al. 2012) and Aguapanela Perafán, Cifuentes \& Estrada-Gomez, 2015 (Perafán et al. 2015).

\section{Description}

Male (holotype, IBSP 107411)

Color in life carapace and dorsal face of abdomen dark brown, ventral side lighter brown, legs covered with reddish hairs. In ethanol: carapace reddish-brown as dorsal face of abdomen, ventral side and legs light brown.

Total length 27. Carapace 11 long, 13 wide. Clypeus 0.2 . Eye tubercle 1.6 long, 2.2 wide. AME 0.2 long, 0.3 wide; PME 0.2 long, 0.2 wide; ALE 0.4 long, 0.4 wide; PLE 0.3 long, 0.2 wide. Basal segment of chelicerae with 10 teeth. Labium 2.1 long, 1.7 wide, with 115 pointed cuspules. Endites with 115-117 

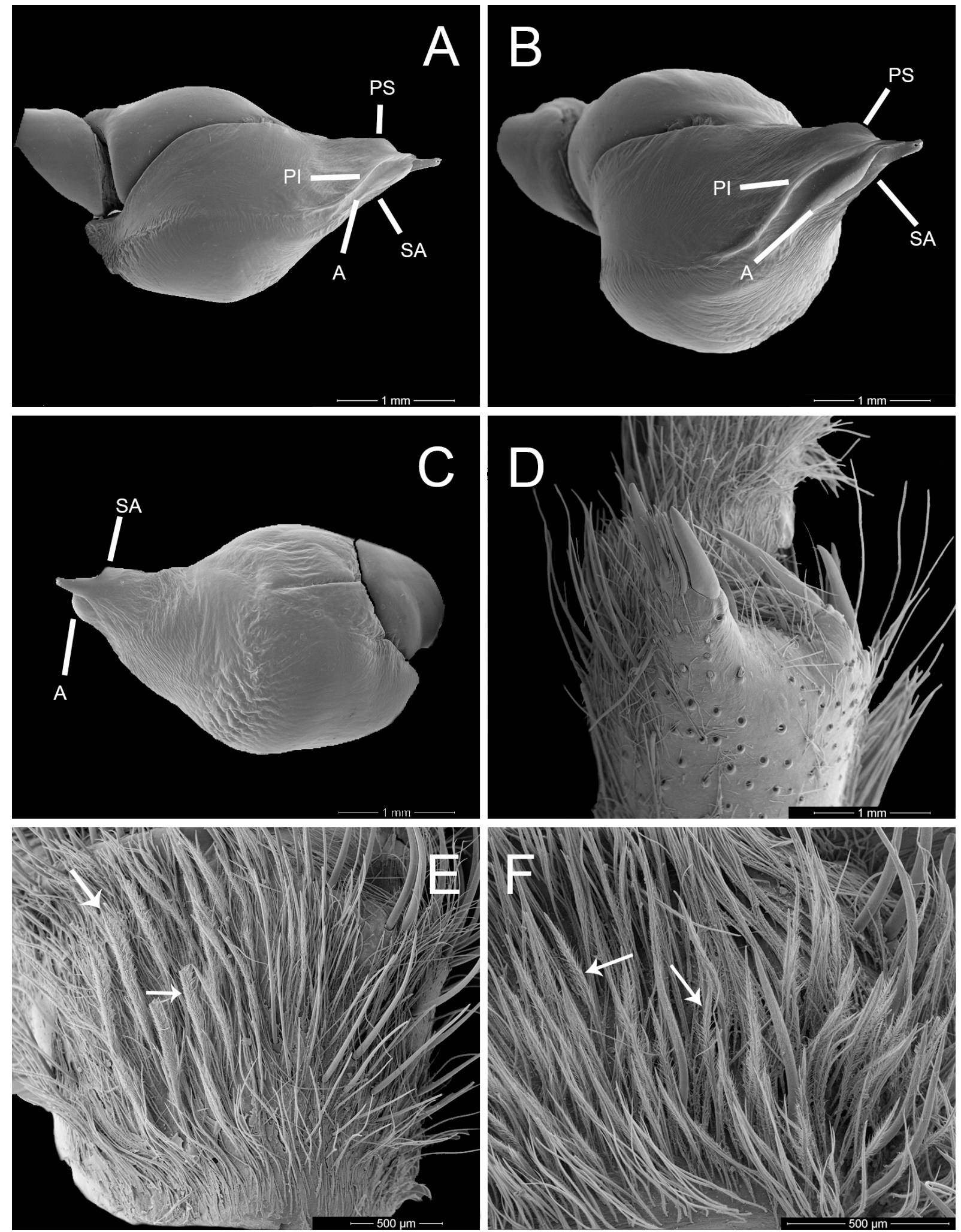

Fig. 8. Umbyquyra paranaiba gen. et sp. nov. Holotype, §ิ (IBSP 107411). A-C. Palpal bulb. A. Prolateral. B. Anterolateral. C. Retrolateral. D. Tibial apophysis, retrolateral. E. Stridulatory organ, trochanter, male palp. F. Stridulatory organ, trochanter I (arrows in E-F pointed the stridulatory bristles). Abbreviations: see Material and methods. 
pointed cuspules. Stridulatory bristles with around 15 bristles on palp trochanter and 45 on leg I. Palp: femur 7.8, patella 4.3, tibia 7.1, tarsus 3.4, total 22.6. Leg I femur 14, patella 6.5, tibia 10.7, metatarsus 11.5, tarsus 6.7, total 49.4. II 13, 6.1, 9.8, 9.8, 6.2, 44.9. III 12, 5.5, 8.5, 10.3, 6.3, 42.6. IV 14, 6.5, 11.2, 17, 6.3, 54.5. Spination: palp: femur d0-0-1-0ap, patella p0-1-0-1-0ap tibia d0-0-1app, v0-1-21p-1 p-1r-0-0ap, p0-1-1-1-1-2-1-1-2-1-2-1 ap, r0-0-1 ap. Legs I femur d0-0-1 app, patella p0-1-0ap, tibia v0-2-1r1r-1apr-1ap-1app, p0-1-0-1ap, metatarsus v0-1-0-0-1ap. II femur d0-0-1app, patella d 0-1r-0ap, p0-1-0ap, tibia v0-1r1p-0-1-1p-1p-1r-0-1apr, p0-1-0-1-0ap, metatarsus d0-1r-0-0ap, v0-1p-0-1r-0-02app-1ap-1 apr. III femur d0-0-1apr, p0-0-1-0ap, patella d0-1r-0-0-0ap, tibia v0-1-2-1r-1-1-1apr-2ap1app, p0-0-1-1-0ap, r2-1-1-1-1-2ap, metatarsus d0-0-1r-1p-0-1r-1p-0ap, v1r-1p-1p-1r-0-1apr-1ap-1app, p0-0-1-0-0-1-0ap, r1-1-1-1-1-3ap. IV femur d0-0-1apr, tibia v0-0-1-1r-2-0-2apr-1ap-1app, p0-1-0-01ap, r0-1-0-1-1ap, metatarsus d0-0-1r-0-0ap, v, 1r-1p-1r1-1r-1r-1p-2-2-1r-1p-1r-1p-2app-1ap-1app, r10-0-1-0-0-1ap. Palpal bulb with short embolus and four keels: PS, PI, A, SA. Tibial apophysis of leg I: retrolateral branch and two strong distal spines with three median setae (Figs 7C, 8D). PLS: basal, median and apical segments 2.2, 2, 2.2 long, respectively.

Female (paratype IBSP 111695)

In ethanol, color as in male. Total length 32. Carapace 13 long, 9.5 wide. Clypeus 0.3 . Eye tubercle 1.5 long, 2 wide. AME 0.4 long, 0.4 wide; PME 0.4 long, 0.3 wide; ALE 0.2 long, 0.3 wide; PLE 0.3 long, 0.2 wide. Basal segment of chelicerae with eight teeth. Labium 1.6 long, 1.8 wide, with 120 pointed cuspules. Endites with 115-117 pointed cuspules. Stridulatory bristles as in male. Palp: femur 6, patella 2, tibia 3, tarsus 2.5, total 13.5. Leg I femur 8, patella 3.5, tibia 5, metatarsus 4, tarsus 2, total 22.5 . II 7, 3, 3.5, 4.5, 2, 20. III 6, 2, 3, 5, 2.2, 27.7. IV 9, 2.5, 5.2, 9.2, 2.5, 28.4. Spination: palp: femur p0-01app, tibia v0-1r-1 apr-1 ap-2app, p0-0-1 ap. Leg I tibia v0-1r-0ap, r0-0-1-0ap, metatarsus v0-1-0-0-1ap. II tibia 1-0-1-1-1-0-2ap, metatarsus v0-1-1-0-0-1ap. III tibia v0-1-1app, p0-1-0-1-0-1ap, 0-1-1-1-0-11ap, metatarsus v1-1r-1r-1p-0-0ap, r2-0-1-0-1 ap. IV tibia v0-0-1-1-1ap-1app, p0-1-0-1ap-r0-1-0-1-0ap, metatarsus d0-1r-0-0-1ap, v1-1p-1-1-1-1p-1p-1-2ap, p0-1-0-0ap, r0-0-1-1ap. PLS basal, median and apical segments 2.7, 1.9, 2.7 long, respectively. Spermathecae with RS rounded at apex, distant from each other by approximately its diameter (Fig. 7D).

\section{Distribution}

Umbyquyra paranaiba gen. et sp. nov. has the widest distribution, occurring in the following Brazilian states: Acre, Mato Grosso, Mato Grosso do Sul and São Paulo (Fig. 29).

Umbyquyra sapezal gen. et sp. nov. urn:1sid:zoobank.org:act:291244D4-A64B-4204-8A63-2038F2357CE5

Figs 5B, 9, 10, 30

\section{Diagnosis}

Males of Umbyquyra sapezal gen. et sp. nov. have a strong procurve projection of the cephalic region resembling those of $U$. palmarum gen. et comb. nov. but differ by the two long stretch marks (Fig. 5B) and palpal bulb with the most enlarged A and SA keels (Figs 9A, 10A-B).

\section{Etymology}

The species epithet is a noun in apposition taken from the type locality.

\section{Type material}

\section{Holotype}

BRAZIL: đ’, Mato Grosso, Sapezal, Usina Hidrelétrica de Sapezal, 13³2'55.2" S, 5847'27.2" W, 2007, F.D. de Godoy leg. (IBSP 113753). 
Paratypes

BRAZIL: $1 \precsim$ (IBSP 113751), $2 \precsim ð$ (IBSP 113752), same data as for holotype.

\section{Description}

Male (holotype IBSP 13753)

In ethanol: same color as U. paranaiba gen. et sp. nov. Total length 30. Carapace 15 long, 12.8 wide. Clypeus 0.3. Eye tubercle 1.2 long, 1.8 wide. AME 0.35 long, 0.3 wide; PME 0.25 long, 0.2 wide; ALE 0.45 long, 0.25 wide; PLE 0.35 long, 0.25 wide. Basal segment of chelicerae with 11 teeth. Labium 2 long, 2.2 wide, with 97 cuspules. Endites with 130-132 cuspules. Stridulatory bristles with around 15 bristles on palp trochanter and $45-50$ on leg I (Fig. 10E-F). Palp: femur 8, patella 3.7 tibia 8.2 , tarsus 2.8, total 22.7. Leg I femur 13.4, patella 5.6, tibia 11, metatarsus 11 , tarsus 6.6 , total 47.6. II 13, 4, 10 , 10.2, 6.3, 43.5. III 10.6, 3.4, 8.3, 11, 6.6, 60. IV 13.5, 4.8, 12, 15.8, 6.7, 52.8. Spination: palp: femur d00-1app, tibia d0-0-0-1p-1p-2p-2app, v0-0-1-1-0ap, r0-0-1-1ap. Legs I femur d0-0-1app, tibia v1-1-1r1r-1-1-1p-1r-1p-1app-2apr, r1-0-1-0-1-0-0ap, metatarsus d0-0-1app. II femur 0-0-0-1app, tibia v1-1r-21-1-1 ap, metatarsus v0-1-1-0-0-1 apr, p0-1-0-0ap. III femur d0-0-1apr, tibia v1-1-1-0-0ap, p0-0-1-0ap, r0-1-1-0ap, metatarsus v0-1-1-0-1ap, p1-0-0-1-1-1ap, r0-1-0-0-1ap. IV tibia v0-1-1ap-1r-1apr, p0-11ap, r0-1-0-1ap, metatarsus v1-1r-1-1p-1r-1r-1p-1app-3apr, p1-0-1-0-0ap, r0-1-0-1ap. Tibial apophysis of leg I: retrolateral branch with two strong distal spines with 3-6 median setae; prolateral branch with
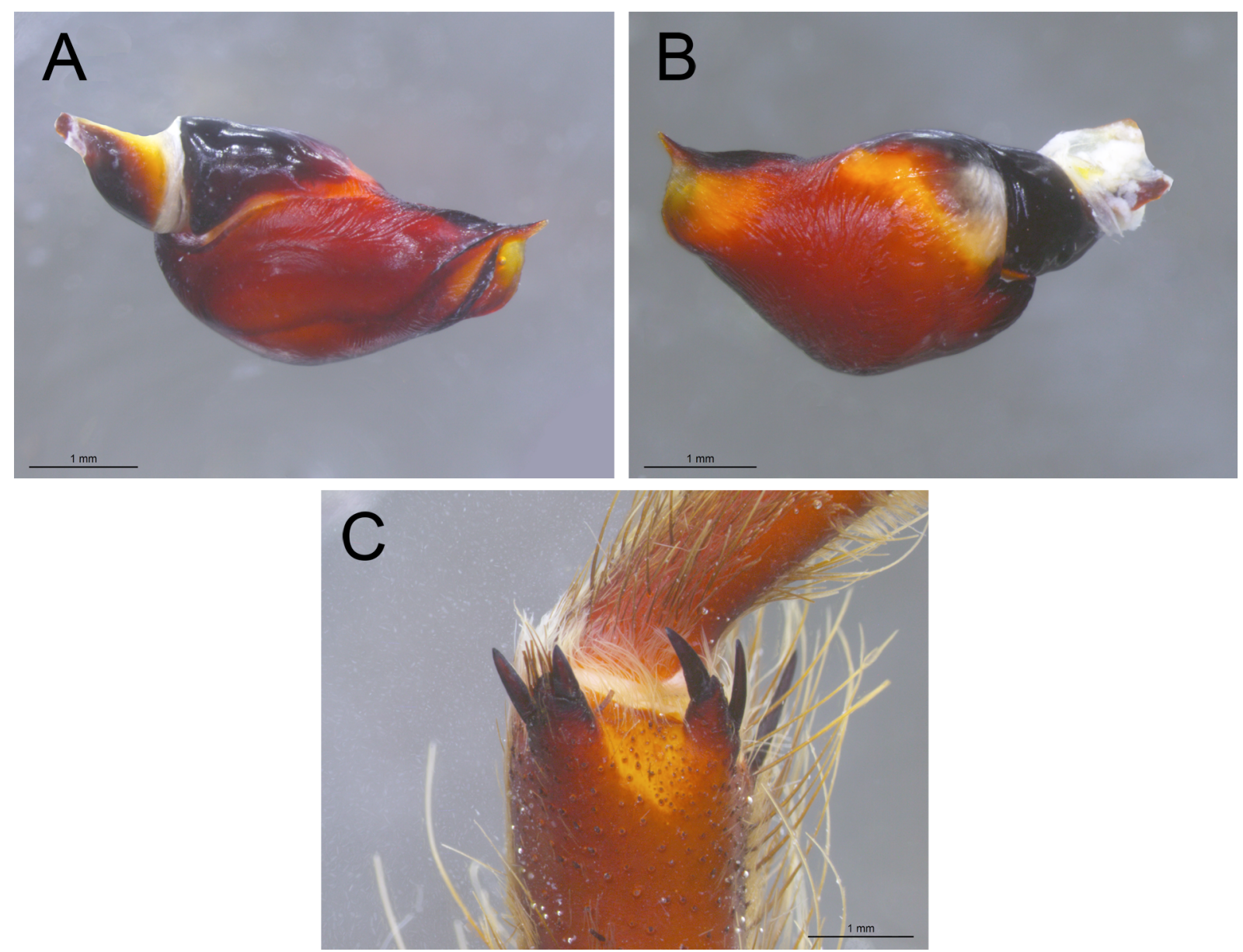

Fig. 9. Umbyquyra sapezal gen. et sp. nov., holotype, $\widehat{\jmath}$, Sapezal, Mato Grosso (IBSP 113753). A. Palp prolateral. B. Retrolateral. C. Tibial apophysis, retrolateral. 

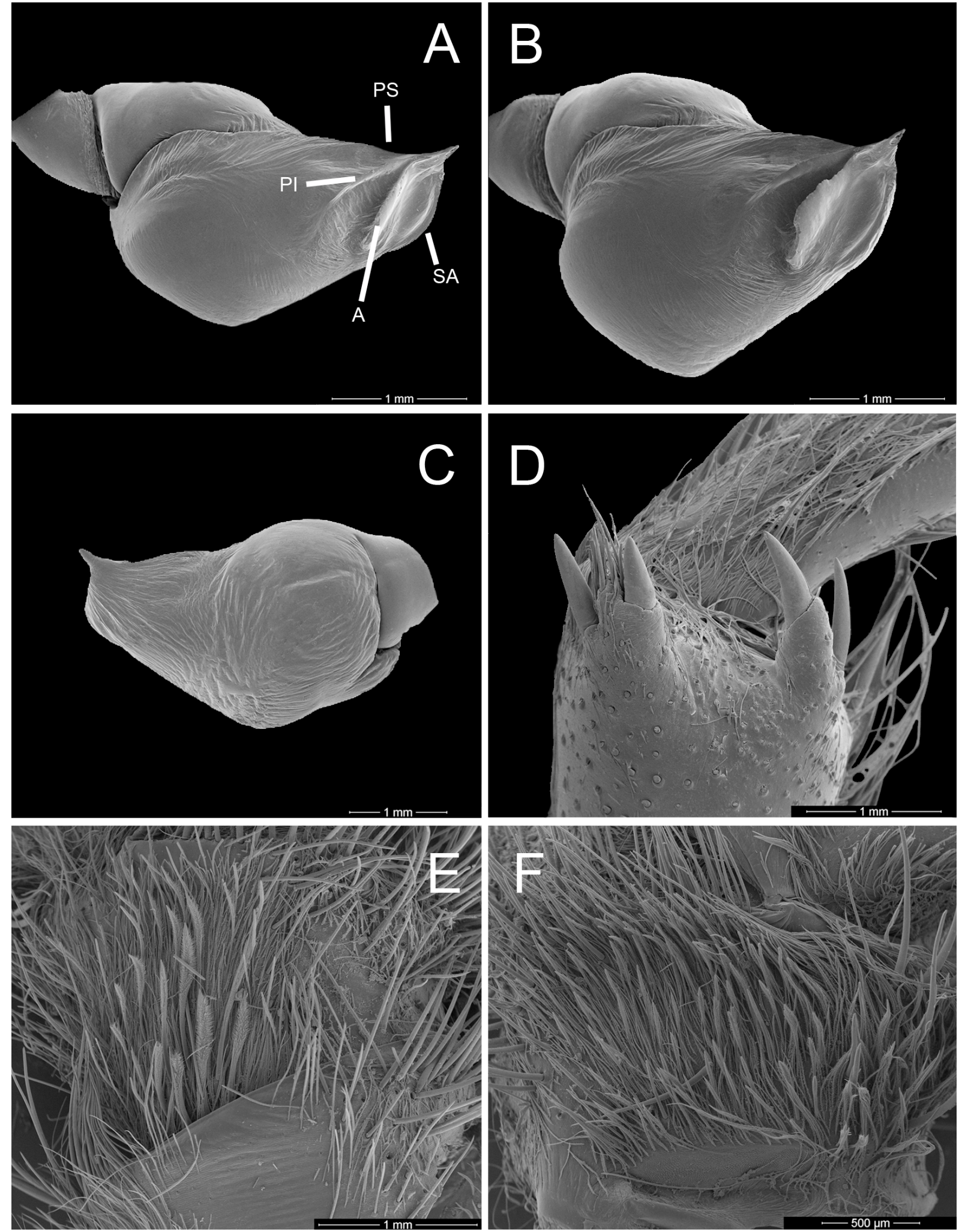

Fig. 10. Umbyquyra sapezal gen. et sp. nov., holotype, $\widehat{\partial}$, Sapezal, Mato Grosso (IBSP 113753). A. Palp prolateral. B. Anterolateral. C. Retrolateral. D. Tibial apophysis, retrolateral. E. Stridulatory organ, palp trochanter. F. Trochanter I. Abbreviations: see Material and methods. 
one distal and one sub basal spines (Figs 9C, 10D). PLS basal, median and apical segments 2.1, 2.1, 2.8 long, respectively.

\section{Female}

Unknown.

\section{Distribution}

Brazil: state of Mato Grosso (Fig. 30).

Umbyquyra palmarum (Schiapelli \& Gerschman, 1945) gen. et comb. nov.

$$
\text { Figs } 5 \mathrm{C}, 11,12,30
$$

Cyrtopholis palmarum Schiapelli \& Gerschman, 1945: 182, pl. IX.

Cyrtopholis palmarum - Gerschman \& Schiapelli 1973: 68, figs 1-5. — Schmidt 1993: 63, figs 68-69; 1997: 18, 149-150.

Acanthoscurria palmarum - Schmidt 2003: 133, 142, figs 170-171.
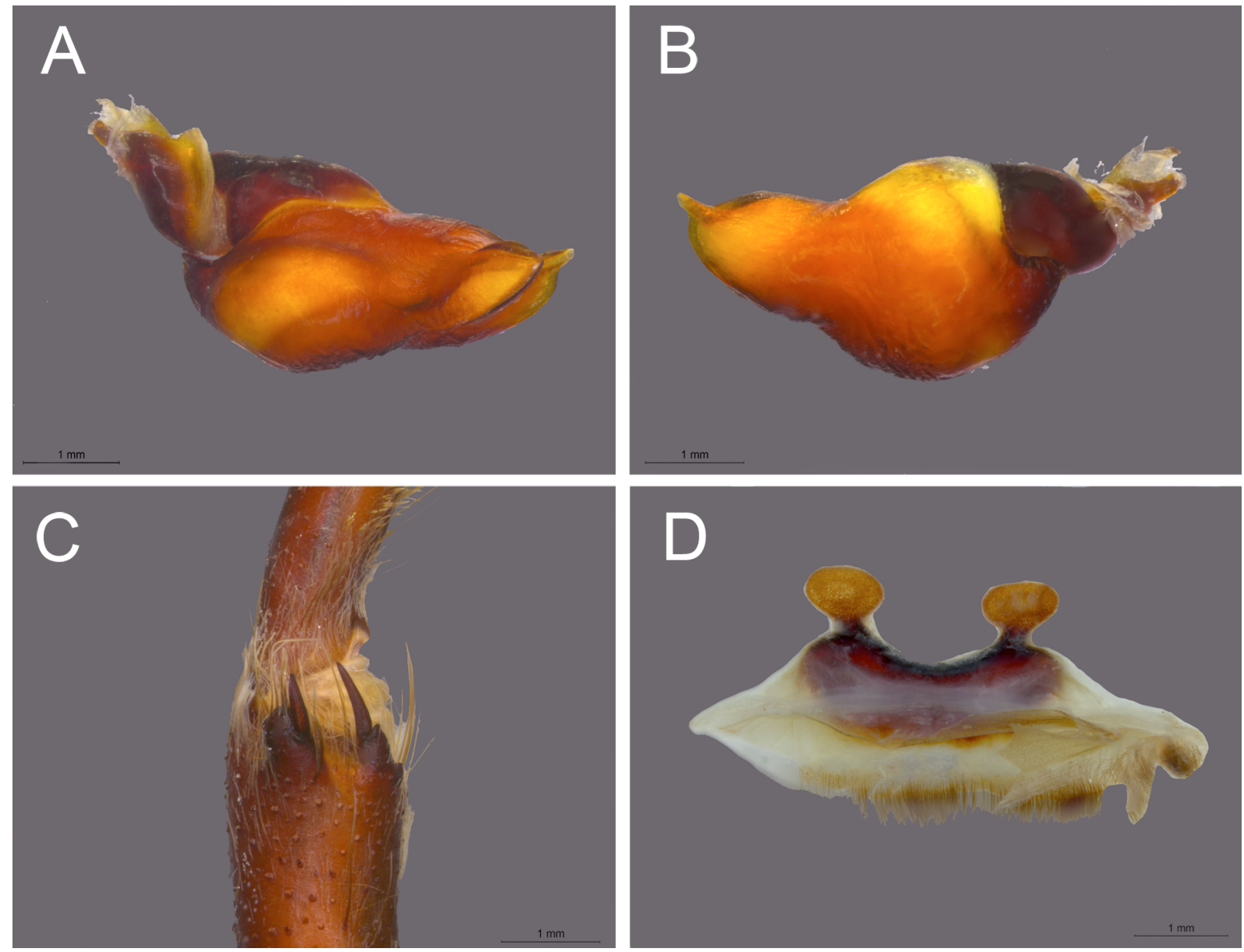

Fig. 11. Umbyquyra palmarum (Schiapelli \& Gerschman, 1945) gen. et comb. nov. A-C. Holotype, đ̊̃, Barão de Melgaço River, Rondônia, Brazil (MACN 832). A. Palp prolateral. B. Retrolateral. C. Tibial apophysis, retrolateral. D. +, Chapada dos Guimarães, Mato Grosso, Brazil (IBSP 107257), spermathecae, dorsal. 

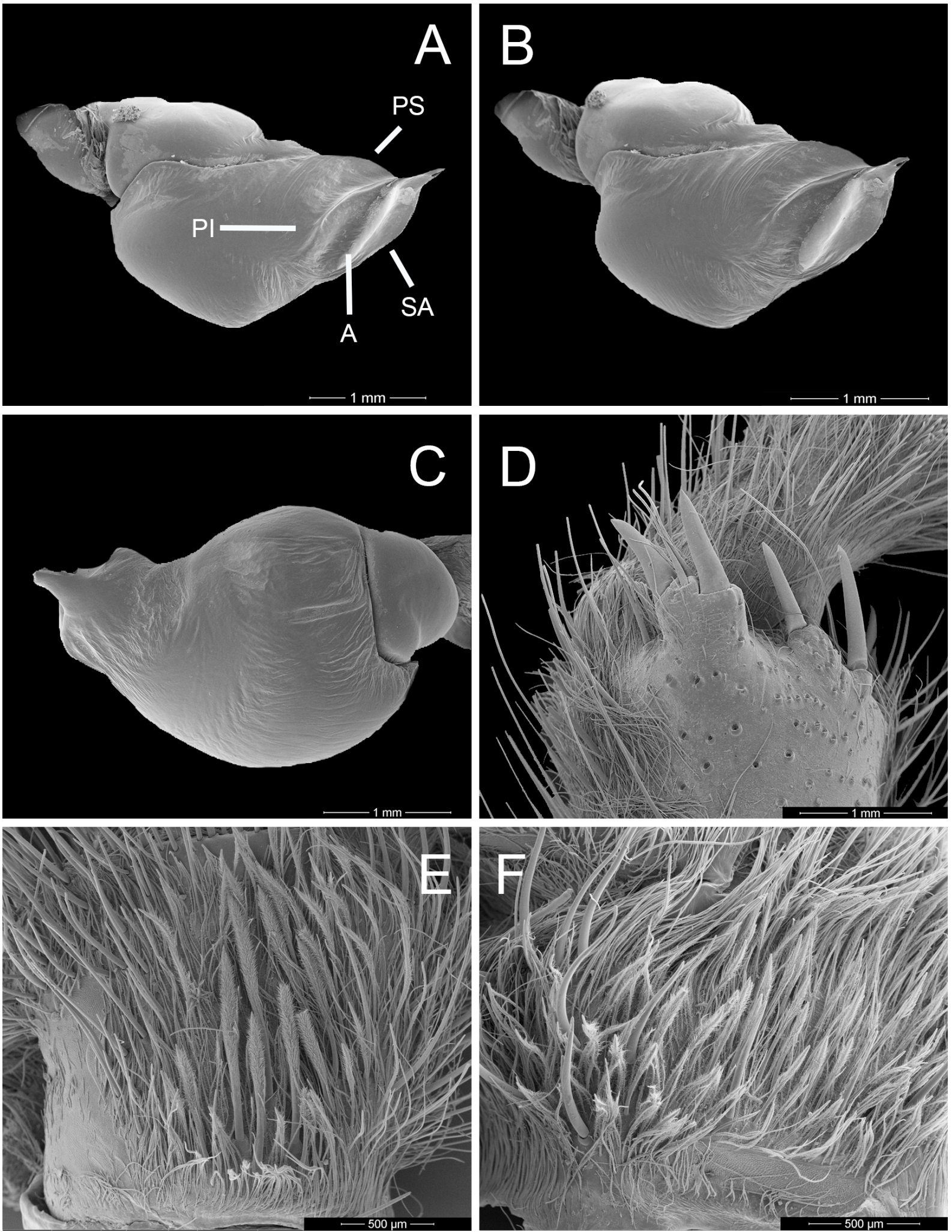

Fig. 12. Umbyquyra palmarum (Schiapelli \& Gerschman, 1945) gen. et comb. nov. Ô, Canoa Quebrada, Mato Grosso, Brazil (IBSP 167427). A. Palp prolateral. B. Anterolateral. C. Retrolateral. D. Tibial apophysis, retrolateral. E. Stridulatory organ, palp trochanter. F. Trochanter I. Abbreviations: see Material and methods. 
GARGIULO F. de F. et al., Umbyquyra gen. nov., new genus of Neotropical Theraphosidae

\section{Diagnosis}

Males and females of $U$. palmarum gen. et comb. nov. differ from those of the other species by the strongly procurved fovea, like a half moon, delimitating the very long projection of the cephalic region (Fig. 5D) and male palpal bulb with well-developed A and SA keels (Figs 11A, 12A-B). Female spermathecae with antero-medially excavated base and SR with very short ducts (Fig. 11D).

\section{Type material}

\section{Holotype}

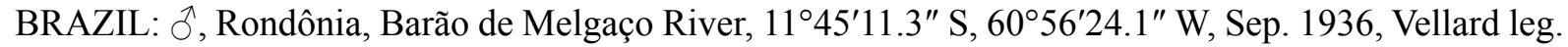
(MACN 832).

\section{Additional material}

BRAZIL: Mato Grosso: 1 q, Chapada dos Guimarães, $15^{\circ} 27^{\prime} 46.9^{\prime \prime}$ S, $55^{\circ} 44^{\prime} 59.2^{\prime \prime}$ W, 1982. R.R. da Silva leg. (IBSP 107257); $2 \jmath^{\lambda}{ }^{\circ}$, Lucas do Rio Verde, PCH Canoa Quebrada, $13^{\circ} 04^{\prime 2} 24.9^{\prime \prime} \mathrm{S}, 55^{\circ} 55^{\prime} 11.1^{\prime \prime} \mathrm{W}$,

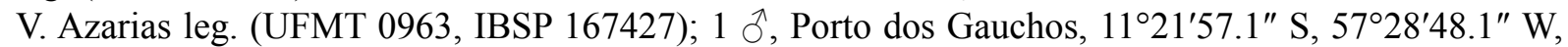
31 Aug. 1962, H. Schulze leg. (IBSP 103628).

\section{Description}

Male (holotype, MACN 832)

In life: according to Vellard (1945), reddish brown color with golden bristles covering all dorsal face of body. In ethanol: same color as U. paranaiba gen. et sp. nov. Total length 23. Carapace 8.8 long, 8.4 wide. Clypeus 0.2. AME 0.37 long, 0.35 wide; PME 0.25 long, 0.2 wide; ALE 0.25 long, 0.25 wide; PLE 0.35 long, 0.22 wide. Basal segment of chelicerae with 10 teeth. Labium 1.3 long, 1.8 wide, with 120 cuspules. Endites with 135-132 cuspules. Eye tubercle: 1.2 long, 2.0 wide. Stridulatory bristles with around 15 bristles on palp trochanter and 45 on leg I (Fig. 11E-F). Palp: femur 8.5, patella 2.6, tibia 6.9, tarsus 2.3 , total 20.3. Leg I femur 12.3, patella 4.5, tibia 11, metatarsus 9.6, tarsus 5.6, total 43. II 10.8, 3.5, 9, 9, 5.2, 37.5. III 9.6, 3.3, 8, 10.2, 4.4, 35.5. IV 12.3, 4, 11, 14.5, 5.6, 47.4. Spination: palp: femur d0-0-1app, tibia d0-1p-2p-2p-1app, v0-0-2p-0ap, p0-0-1-2-2-1-1-1-11ap. Legs I femur d0-0-1 app, tibia v1-0-1-1-1-1 ap-1 app, p1-0-1-0ap, metatarsus v-0-0-0-1 ap. II femur d0-0-1 app, tibia d0-1p-0-1p-1p-0-1 app, v1-0-1-2-1ap, metatarsus v0-1-0-1-0-0-2ap-1app. III tibia v01-1r-1 apr-1 ap-1 app, p0-2-0-1 ap, r1-1-1ap, metatarsus v1-1-0-1-2ap, p1-1-1-1-0-2ap, r0-1-0-1-1 ap, r01-0-1 ap. IV tibia d0-1r-0-1r-0-1apr, v0-2-1-1r-1-1 apr-2app, p0-1-1-0ap, metatarsus d0-1r-0app, v11-1p-2r-1r-1-1p-2-1r-1p-1p-1r-2app-1ap, p0-0-1-0-1ap. Tibial apophysis of leg I: retrolateral branch well-developed with two strong distal spines and two median setae; prolateral branch weakly projected with distal spine and sub basal spine (Figs 11C, 12D). PLS basal, median and apical segments 2.3, 1.7, 2.0 long, respectively.

\section{Female (IBSP 107257)}

In ethanol: same as U. paranaiba gen. et sp. nov. Total length 40.0. Carapace 13.5 long, 12.5 wide. Clypeus 0.3. AME 0.35 long, 0.3 wide; PME 0.2 long, 0.2 wide; ALE 0.25 long, 0.45 wide; PLE 0.3 long, 0.3 wide. Basal segment of chelicerae with 11 teeth. Labium 2.1 long, 2.7 wide, with 125 cuspules. Endites with 140-142 cuspules pointed. Eye tubercle: 1.9 long, 2.4 wide. Stridulatory bristles as in male. Palp: femur 9, patella 5.3, tibia 6.9, tarsus 5.8, total 27. Leg I femur 13.4, patella 7.4, tibia 9.4, metatarsus 4.2, tarsus 5.4, total 39.8. II 12, 6.5, 6.5, 6.4, 7.2, 35.7. III 10.3, 7.2, 6.2, 9.1, 5.1, 37.9. IV 12.8, 6.3, 8.8, 10, 5.1, 43. Spination: palp: tibia v0-0-0-1p-1r-3ap, p0-0-1-1ap. Legs I femur d00-1apr, tibia v0-1-2app, r0-1-0-3ap, metatarsus v-0-1ap, r0-1ap. II femur d0-0-1apr, tibia v0-1-0-3ap, p0-1-0-1ap, metatarsus v0-1-0-0-2apr. III femur 0-0-1apr, tibia v0-3-0-1app-1ap-1apr, p0-2-0-0ap, r11-1ap, metatarsus d1-2p-0-2ap, v1-1r-1p-0-1ap, p1-1p-1p-1r-1r-1-1ap. IV femur 0-0-1app, tibia v0-1p2-1-1ap, 0-1-2ap, r0-1-0ap, metatarsus v1r-1r-2p-0-1p-1p-1p-1r-1r-1-2p-2ap, 0-0-1-0ap, p0-0-1-0ap, 
r0-1-0-1ap. PLS basal, median and apical segments 2.1, 2.6, 3.5 long, respectively. Spermathecae with RS rounded, apart from each other by approximately its own diameter (Fig. 11D).

\section{Distribution}

Brazil: states of Rondônia and Mato Grosso (Fig. 30).

\section{Natural history}

Jean Vellard, in 1945, collected two males in a rotten palm tree. According to the author, the spiders were very aggressive and no webs in the rotten palm tree, were was found.

Umbyquyra schmidti (Rudloff, 1996) gen. et comb. nov.

Figs 5, 13, 14, 31

Cyrtopholis schmidti Rudloff, 1996: 3, figs 1-2.

Acanthoscurria schmidti - Schmidt 2003: 143, fig. 345.
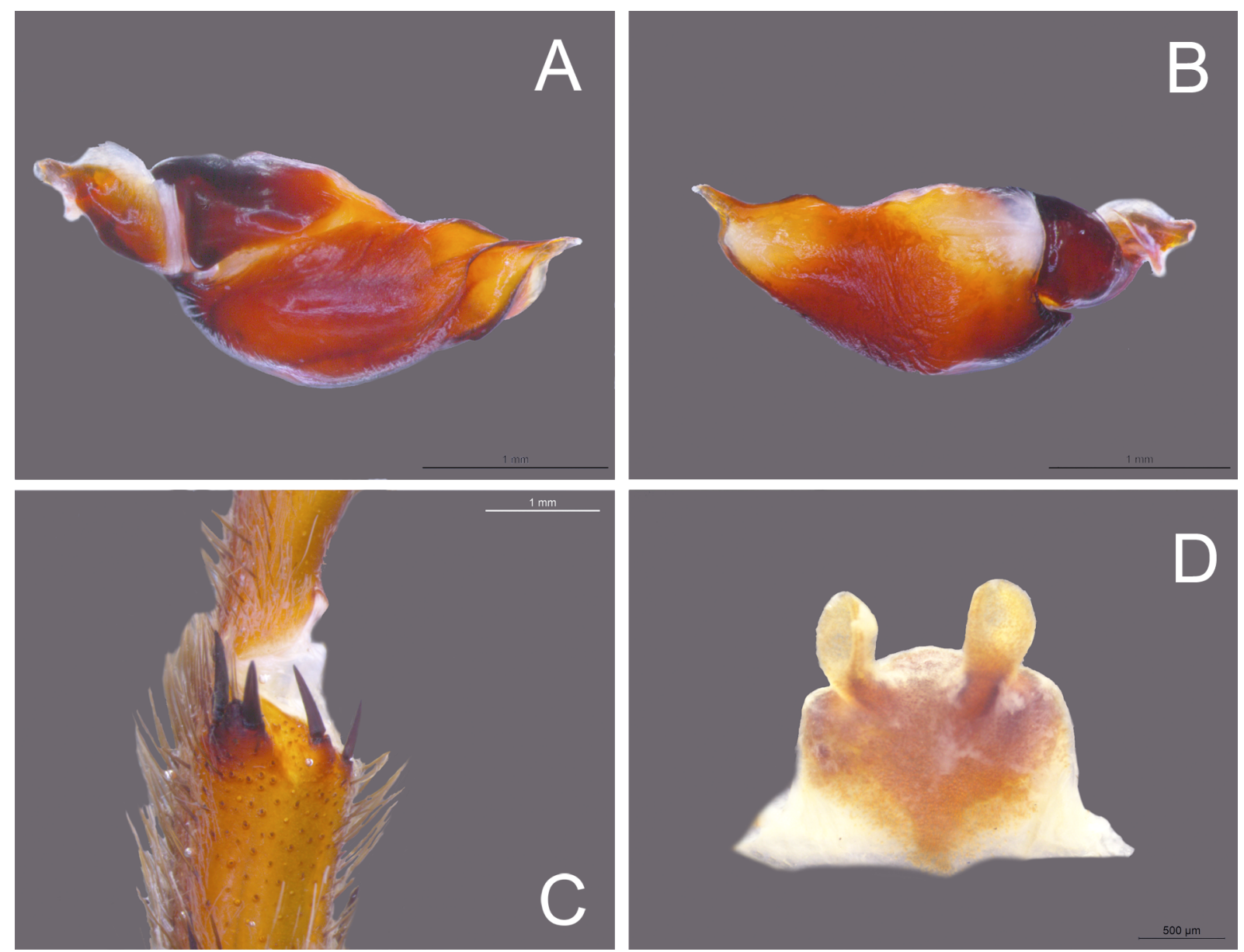

Fig. 13. Umbyquyra schmidti (Rudloff, 1996) gen. et comb. nov. A-C. ̊̊, Cuiabá, Mato Grosso, Brazil (IBSP 110728). A. Palp prolateral. B. Retrolateral. C. Tibial apophysis, retrolateral. D. $\odot$, spermathecae, dorsal (IBSP 107159). 


\section{Diagnosis}

Males and females of $U$. schmidti gen. et comb. nov. differ from those of the other species by a carapace with large procurve fovea and a cephalic region projected over the same (Fig. 5A), less than 15 stridulatory bristles on palp trochanter, less than 30 stridulatory bristles on leg I (Fig. 14E-F), conspicuous SA and A (Fig. 13A-B) and tibial apophysis with prolateral reduced branch reduced (Figs 13C, 14D). Females differ from those of the other species by spermathecae with a cordiform basal area and SR close to each other emerging from the middle basal area (Fig. 13D).

\section{Material examined}

\section{Type material}

\section{Holotype}

BRAZIL: + , São Paulo, south of the city of São Paulo (no coordinates), Sep. 1995, H.U. Rechsteiner leg., examined by photos (ZMB 30790).

\section{Additional material}

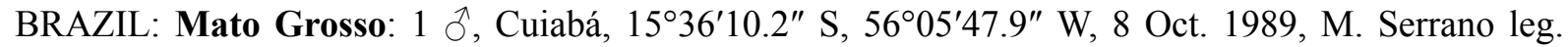
(IBSP 110728); 1 ${ }^{\lambda}$, same collection data as for preceding, Oct. 1972, G.R. Kloss and F. Val leg. (MZSP

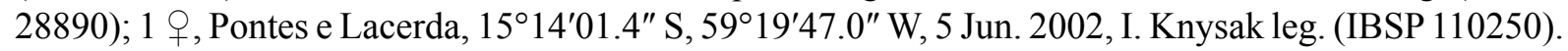

\section{Description}

Male (IBSP 110728)

In ethanol: same color as U. paranaiba gen. et sp. nov. Total length 25. Carapace 7.5 long, 6.7 wide. Clypeus 0.4. AME 0.3 long, 0.33 wide; PME 0.1 long, 0.2 wide; ALE 0.2 long, 0.4 wide; PLE 0.3 long, 0.2 wide. Basal segment of chelicerae with 10 teeth. Labium 1 long, 1.5 wide, with 110 cuspules. Endites with 125-127 cuspules. Eye tubercle: 1.0 long, 1.6 wide. Stridulatory bristles with less than 15 bristles on palp trochanter and less than 30 on leg I (Fig. 14E-F). Palp: femur 6.0, patella 2.2, tibia 5.2, tarsus 2.2, total 15.6. Leg I femur 9.8, patella 2.8, tibia 8.6, metatarsus 7.5, tarsus 4.4, total 33.1. II 7.5, 3.6, 6, 7, 4.1, 28.2. III 7.4, 3.3, 5.4, 8.1, 3.6, 27.8. IV 7, 3.2, 8.3, 10, 6.1, 34.6. Spination: palp: tibia v0-0-0-1p-1r-3ap, p0-0-1-1ap. Leg I femur d0-0-1apr, tibia v0-1-2app, r0-1-0-3ap, metatarsus v-0-1ap, r0-1ap. II femur d00-1 apr, tibia v0-0-1-1r-0-0-1 app, metatarsus v0-1r-1p-1r-0-1 apr-1 ap-1 app. III tibia v0-0-1-1 p-1 ap-1 app, r0-0-1-0ap, metatarsus d0-0-0-1apr, v0-0-1p-0-1apr-1-1app, p0-1-1ap, r0-1-1ap. IV tibia v0-0-1-1r-1r0ap, p0-0-1-0ap, r0-1-0-1-0ap, metatarsus d0-0-1r-1p-0-0ap, v1p-1r-1-1-1p-1r-1-1r-1-1p-1r-1p-1ap, p00-1-0ap. Tibial apophysis of leg I: retrolateral branch large and slightly projected with two strong distal spines and two median setae on projection; prolateral branch inconspicuous with distal and sub distal spines (Figs 13C, 14D). PLS: basal, median and apical segments 2.0, 2.2, 0.9 long, respectively.

\section{Female (IBSP 110250)}

In ethanol: same color as U. paranaiba gen. et sp. nov. Total length 17.8. Carapace 5.7 long, 5.1 wide. Clypeus 0.4. Eyes: anterior row slightly procurved and posterior row recurved. AME 0.38 long, 0.35 wide; PME 0.2 long, 0.15 wide; ALE 0.4 long, 0.3 wide; PLE 0.23 long, 0.3 wide. Basal segment of chelicerae with eight teeth. Labium 1.5 long, 2.0 wide, with 115 cuspules. Endites with 130-134 cuspules. Eye tubercle 1.5 long, 2.0 wide. Stridulatory bristles as in male. Palp: femur 5.8, patella 2.5, tibia 6.4, tarsus 3.3, total 18. Leg I femur 6.7, patella 2.7, tibia 6.3, metatarsus 4, tarsus 3.5, total 23.2. II 6.0, 2.5, 3.7, 3.3, 3.2, 18.7. III 5.4, 1.6, 3.4, 4, 3.2, 17.6. IV 6.4, 2.3, 6, 6.5, 3.5, 24.7. Spination: palp: femur d0-0-1 app, tibia v0-1r-1 apr, r0-0-1ap. Legs I tibia v0-1-0ap, metatarsus v0-1-0-0-0ap. II tibia v01-0ap, metatarsus v0-0-1-0ap. III tibia v0-0-1p-1r-0app, p0-1-1-0-0app, metatarsus d0-0-0-1-1ap, v00-1p-1r-1r-2apr-1app, p1-1-1-0-1 app, r0-0-1-0-1ap. IV tibia d0-0-1p-1r-0-0-1apr-1app, metatarsus d00-1p-1r-0-0-1 apr-1 app, v1-1-1-1-1r-1 p-1-1-1p-1r-1-1r-1ap, 0-0-1-0-0-1app, r0-0-1-0-1ap. PLS basal, median and apical segments 1.6, 1.8, 1.6 long, respectively. Spermathecae with oval RS, apart from each 

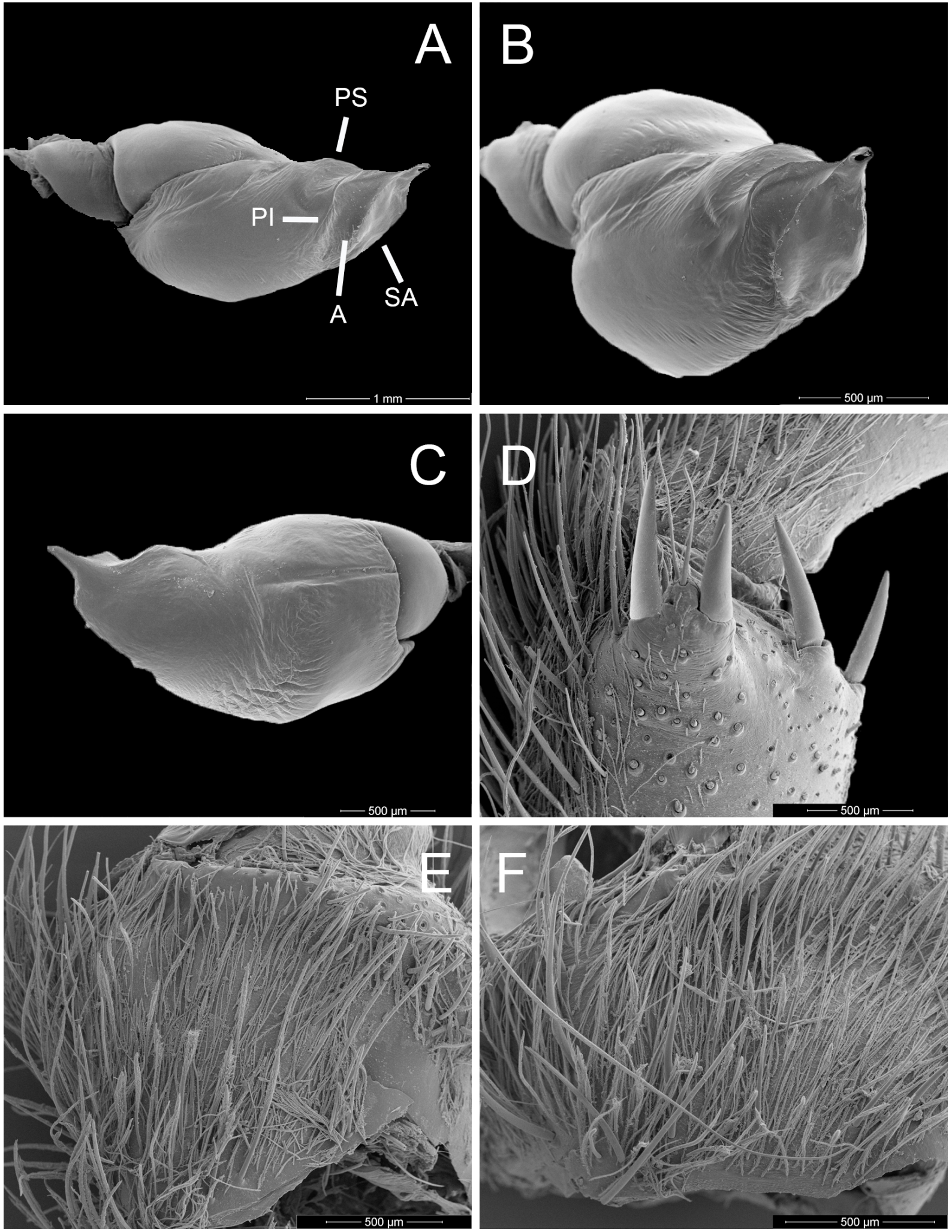

Fig. 14. Umbyquyra schmidti (Rudloff, 1996) gen. et comb. nov., đ̂, Cuiabá, Mato Grosso, Brazil (IBSP 110728), A. Palp prolateral. B. Anterolateral. C. Retrolateral. D. Tibial apophysis, retrolateral. E. Stridulatory organ, palp trochanter. F. Trochanter I. Abbreviations: see Material and methods. 
other by approximately their own diameter, and elongated ducts (Fig. 13D) emerging from a rounded basal area resembling U. caxiuana gen. et sp. nov. but the apex of the SR is not so globular.

\section{Remark}

The other female from the same locality cited in Rudloff (1996), not designated as a type, was not located.

\section{Distribution}

Brazil: states of Mato Grosso and São Paulo (Fig. 31).

Umbyquyra acuminata (Schmidt \& Tesmoingt in Schmidt, 2005) gen. et comb. nov. Figs 15, 18A-B, 30

Acanthoscurria acuminata Schmidt \& Tesmoingt, 2000: 2, figs 1-2. (nom. nud., no type depository). Acanthoscurria acuminata Schmidt \& Tesmoingt in Schmidt, 2005: 4, figs 1-2, 7.

Acanthoscurria acuminata - Schmidt 2000: 1, figs 1-2; 2003: 141, figs 235-238.

\section{Diagnosis}

Males and females of $U$. acuminata gen. et comb. nov. differ from those of the other species by a procurve fovea overlapped by a large projection of the cephalic region (Fig. 15E) and by a retrolateral branch of the tibial apophysis with three strong spines (Fig. 15C). Female spermathecae have a cordiform basal area resembling those of $U$. schmidti gen. et comb. nov., but differs from this species by the RS being more apart when compared to the other species (Fig. 15D).

\section{Material examined}

\section{Holotype}

BOLIVIA: 1 ㅇ, Province Sara, Santa Cruz de la Sierra, 16²7'37.2" S, 63¹0'52.4" W, 1995, Verdez leg. (SMF 3979).

\section{Additional material}

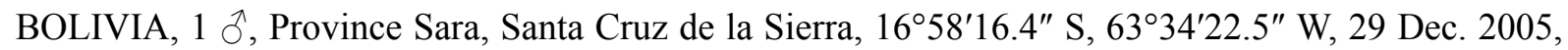
Steinbach leg. (ZMB 47188); 2 q $ᄋ$, same data as for preceding (ZMB 47189, ZMB 47190).

\section{Description}

\section{Male (ZMB 47188)}

In life: dark brown with light brown joints and golden hairs completely covering dorsal side of body (Fig. 16). In ethanol: same color as U. paranaiba gen. et sp. nov. Total length 37.8. Carapace 17.4 long, 15 wide. Clypeus 0.3 . Eye tubercle 2.2 long, 1.7 wide. AME 0.4 long, 0.4 wide; PME 0.28 long, 0.23 wide; ALE 0.4 long, 0.3 wide; PLE 0.4 long, 0.25 wide. Basal segment of chelicerae with 12 teeth. Stridulatory bristles with around 15 bristles on palp trochanter and 45 on leg I (Fig. 17E-F). Palp: femur 8.3, patella 3.7, tibia 8.4, tarsus 3.7, total 24.1. Leg I femur 13.4, patella 4.5, tibia 12.5, metatarsus 12.7 , tarsus 7.8, total 51. II 13.3, 4.6, 11.2, 6, 7.2, 42.3. III 11.6, 3.1, 9.5, 11.5, 6.8, 42.5. IV 15.8, 5.5, 12.8, 16.8, 7.5, 58.4. Spination: palp: tibia v0-1p-0ap, r0-1-2-1-2-1-1-1ap. Leg I femur d0-0-1app, patella v0-1-1-1-0ap, tibia d0-1p-0-0ap, v2-1r-1-0-1-1-1-1r-1-2apr, p0-0-1-0ap, r0-0-0-1-0ap, metatarsus v01r-0-0ap. II femur, 0-0-0-1app, tibia v3-1r-1-1-1-1r-1-0-1apr-1app-1ap, p0-1-0-1-0-1ap, metatarsus v0-1p-1-0-0-0-1 app-1ap-1 apr, p0-0-0ap. III femur d0-0-1 apr, tibia v2-1-0-1-2-1p-1r-0-1apr-1app, p0-11-1-1-1ap, r0-1-0-1-0-0ap, metatarsus v0-0-1p-1r-0-1p-1r-0-2apr-1ap-1app, p2-1-1-1-1-0-0ap, r0-0-10-0ap. IV femur d0-0-1apr, tibia v2-1-1p-1-1-1-2-1r-1 apr-1app, r0-0-1-0ap, metatarsus d0-0-1r-1-1 p-0- 
0ap, r0-0-1-0-0ap, v1p-1r-1r-1-1p-1r-1-1-1p-1-1-1apr-1app. Tibial apophysis of leg I: prolateral branch inconspicuous with distal and sub distal spines. (Fig. 15C). PLS basal, median and apical segments 1.8, $1.1,2.8$ long, respectively.
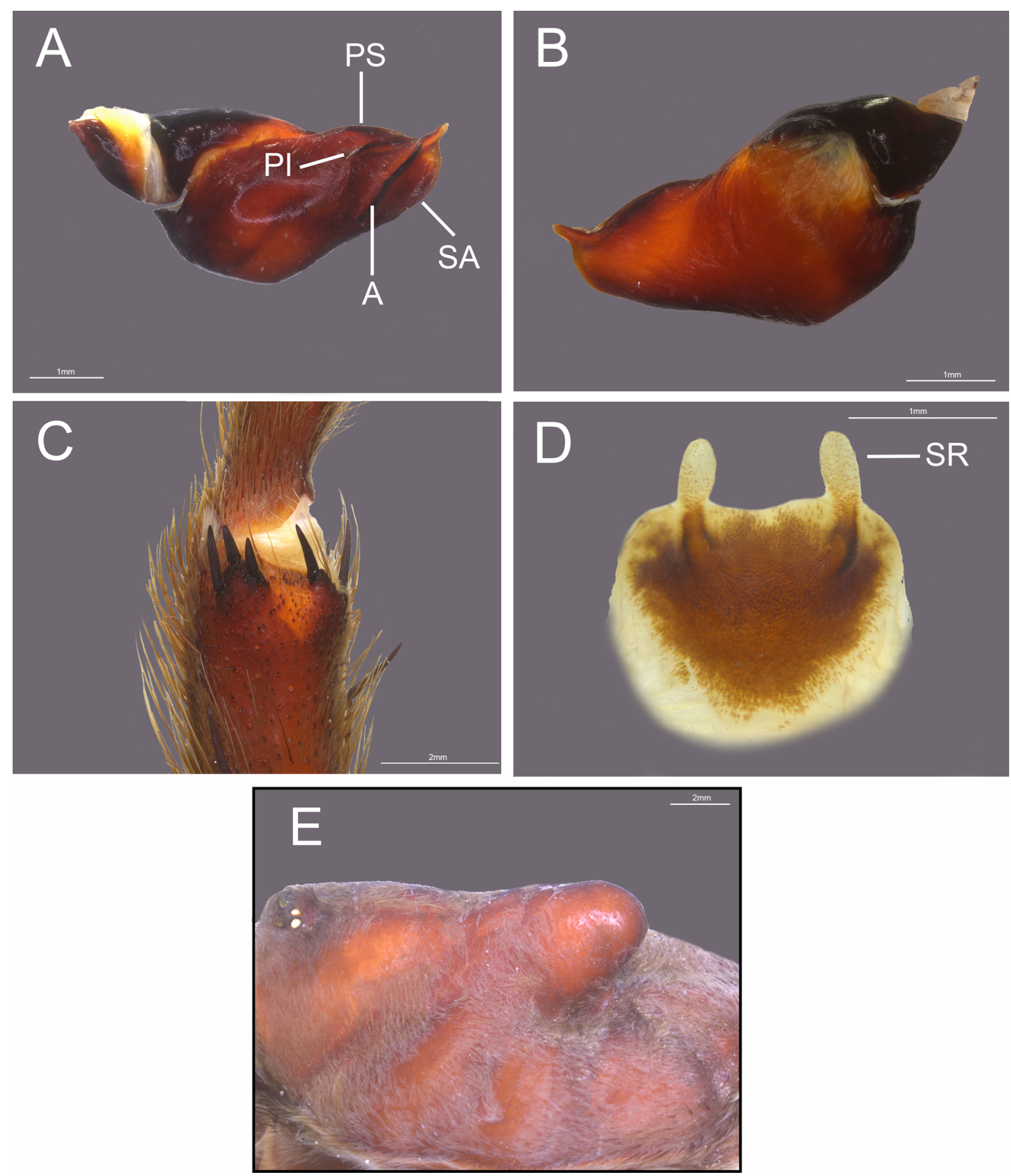

Fig. 15. Umbyquyra acuminata (Schmidt \& Tesmoingt in Schmidt, 2005) gen. et comb. nov. A-C. $\overbrace{}^{\lambda}$, Santa Cruz de la Sierra, Bolivia (ZMB 47188). A. Palp, prolateral. B. Retrolateral. C. Tibial apophysis, retrolateral. D-E. + , same locality (ZMB 47189). D. Spermathecae, dorsal. E. Carapace, lateral. Abbreviations: see Material and methods. 
Female (ZMB 47189)

In ethanol: same color as $U$. paranaiba gen. et sp. nov. Total length 32.2. Carapace 16.6 long, 13 wide. Clypeus 0.3. Eyes: anterior row slightly procurved and posterior recurved. AME 0.4 long, 0.4 wide; PME 0.27 long, 0.27 wide; ALE 0.48 long, 0.33 wide; PLE 0.35 long, 0.22 wide. Basal segment of chelicerae with 12 teeth. 7.0 long, 7.0 wide. Eye tubercle 1.7 long, 2.4 wide. Stridulatory bristles as in male. Palp: femur 7.6, patella 2.7, tibia 6.0, tarsus 5.5, total 21.8. Legs I femur 9.7, patella 3.5, tibia 5.3, metatarsus 4.6, tarsus 4.6, total 27.7. II femur 9.0, patella 3.0, tibia 7.0, metatarsus 5.5, tarsus 3.7, total 28.2. III femur 8.3, patella 3.0, tibia 5.5, metatarsus 6.5, tarsus 4.1, total 27.4. IV femur 8.6, patella 4.0, tibia 4.6, metatarsus 6.4, tarsus 4.2, total 27.8; Spination: palp: tibia v0-0-1p-1apr-2app, r0-0-1-0-1ap. Leg I femur d0-0-1app, tibia v0-1-0-1-1-1-1-0-1 ap-1 app, metatarsus v0-1-1-0-1app-1ap-1 apr. II femur d0-0-1 app, tibia v0-1-0-1-1-1-0-1app-1apr, metatarsus v0-1-1-0-1 app-1ap-1apr. III femur d0-0-1apr, tibia v0-2-0-1-2app-1apr, p0-1-1-0-2ap, metatarsus d0-1p-1r-0-1app-2apr, p0-1-1-1-0-2ap. IV v0-11-0-1 app-2apr, p0-1-0-1ap, r1-1-1-0ap, metatarsus d0-1p-1r-0-1app-2apr, v1-1p-1-1p-1r-0-1apr-1ap1app, p1-1-1-0-1ap, r0-0-0-1 ap. PLS basal, median and apical segments 2.5, 1.4, 2.0 long, respectively. Spermathecae with oval RS and long ducts, apart by approximately twice their diameter (Fig. 15D).

\section{Remark}

Schmidt \& Tesmoingt (2000) described Acanthoscurria acuminata based on a female and a juvenile male and exuvia, respectively from Santa Cruz de la Sierra, Province Sara, Bolívia and Cuiabá, Mato Grosso, Brazil. They presented figures of the carapace with a large projection over the fovea and the aspect of the SR. Later, Schmidt \& Tesmoingt (2005) described another male of this species, collected in Santa Cruz de la Sierra, Bolivia, and deposited it in the regional museum of Santa Cruz. This specimen could not be located. The female holotype is deposited in the SMF and does not have the spermathecae anymore. Moreover, the juvenile male and exuvia could not be located. The species here is transferred to Umbyquyra gen. nov. due to the presence of stridulatory bristles on the palp trochanter and leg I, male palpal bulb with short embolus, four keels and spermathecae emerging from a common base (Fig. 15D).

\section{Distribution}

Brazil: Cuiabá, Mato Grosso; Bolivia: Santa Cruz de la Sierra, Province Sara (Fig. 30).

Umbyquyra cuiaba gen. et sp. nov. urn:1sid:zoobank.org:act:75D2C35B-4325-402E-A169-0748BEB42334

Figs 16, 17, 18C-D, 31

\section{Diagnosis}

Males and females of Umbyquyra cuiaba gen. et sp. nov. resemble those of U. palmarum gen. et comb. nov. by a large procurved fovea delimitating the projection of the cephalic region (Fig. 5D) and by a male palpal bulb with the shape and size of keels, but differ by the tegulum being more compact (Figs 16A-B, 17A-C) and by females having a spermathecae with a very narrow basal area (Fig. 16D).

\section{Etymology}

The species epithet is a noun in apposition taken from the type locality.

\section{Type material}

Holotype

BRAZIL: ${ }^{\wedge}$, Mato Grosso, Cuiabá, $15^{\circ} 36^{\prime} 10.2^{\prime \prime}$ S, 56 $06^{\circ} 47.9^{\prime \prime}$ W, 21 Oct. 2007, M. Shiraiwa leg. (IBSP 167423). 


\section{Paratypes}

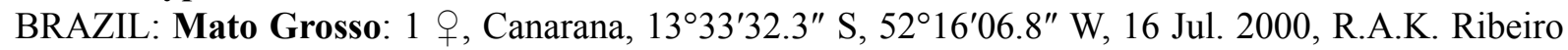

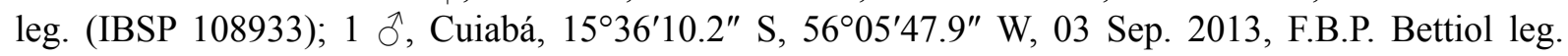
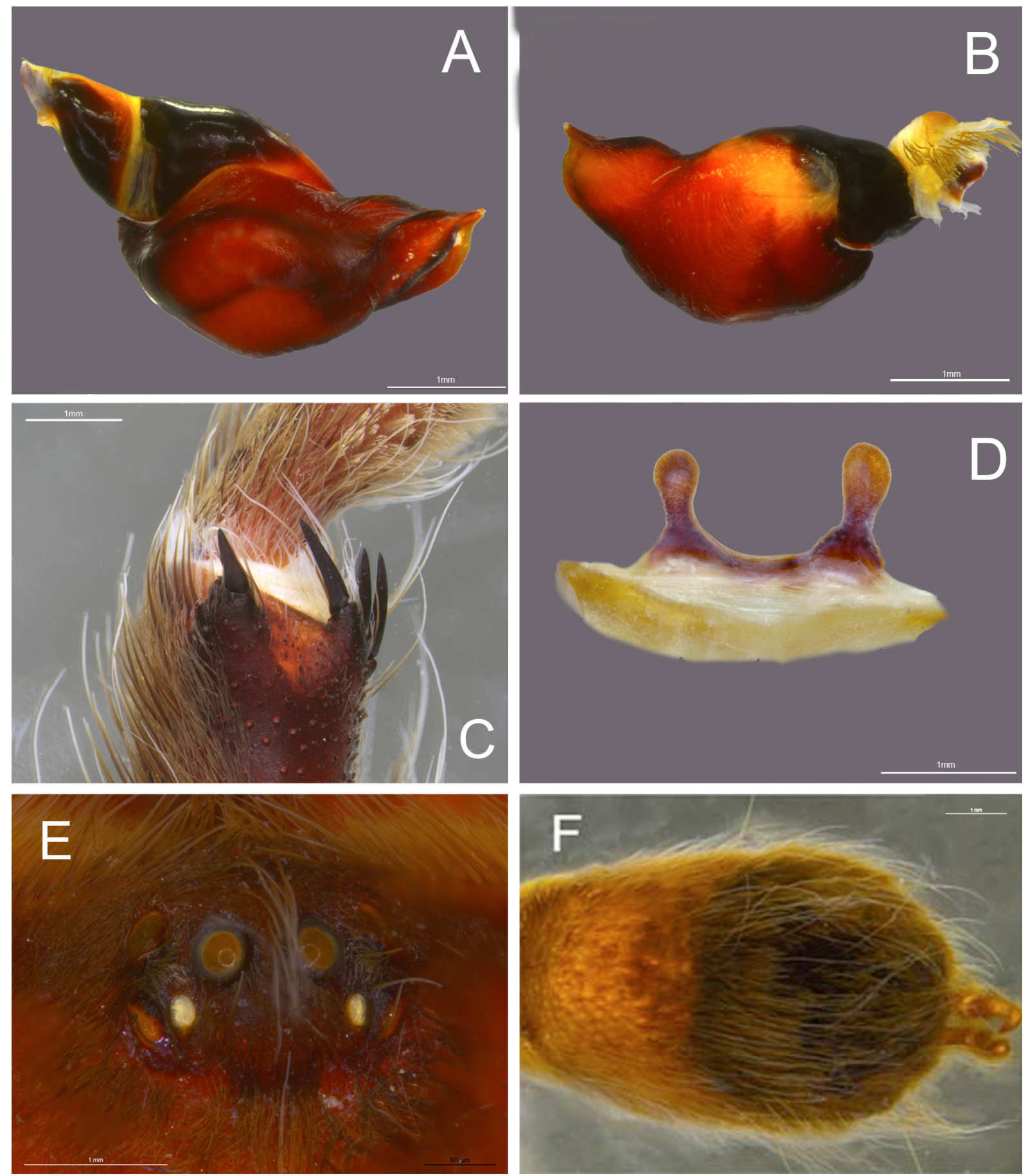

Fig. 16. Umbyquyra cuiaba gen. et sp. nov. A-C. Holotype, ô, Cuiabá, Mato Grosso, Brasil (IBSP 167423). A. Palp prolateral. B. Retrolateral. C. Tibial apophysis, retrolateral. D-F. Paratype, + , Canarana, Mato Grosso, Brazil (IBSP 108933). D. Spermathecae, dorsal. E. Eye tubercle, dorsal. F. Abdomen, dorsal. 

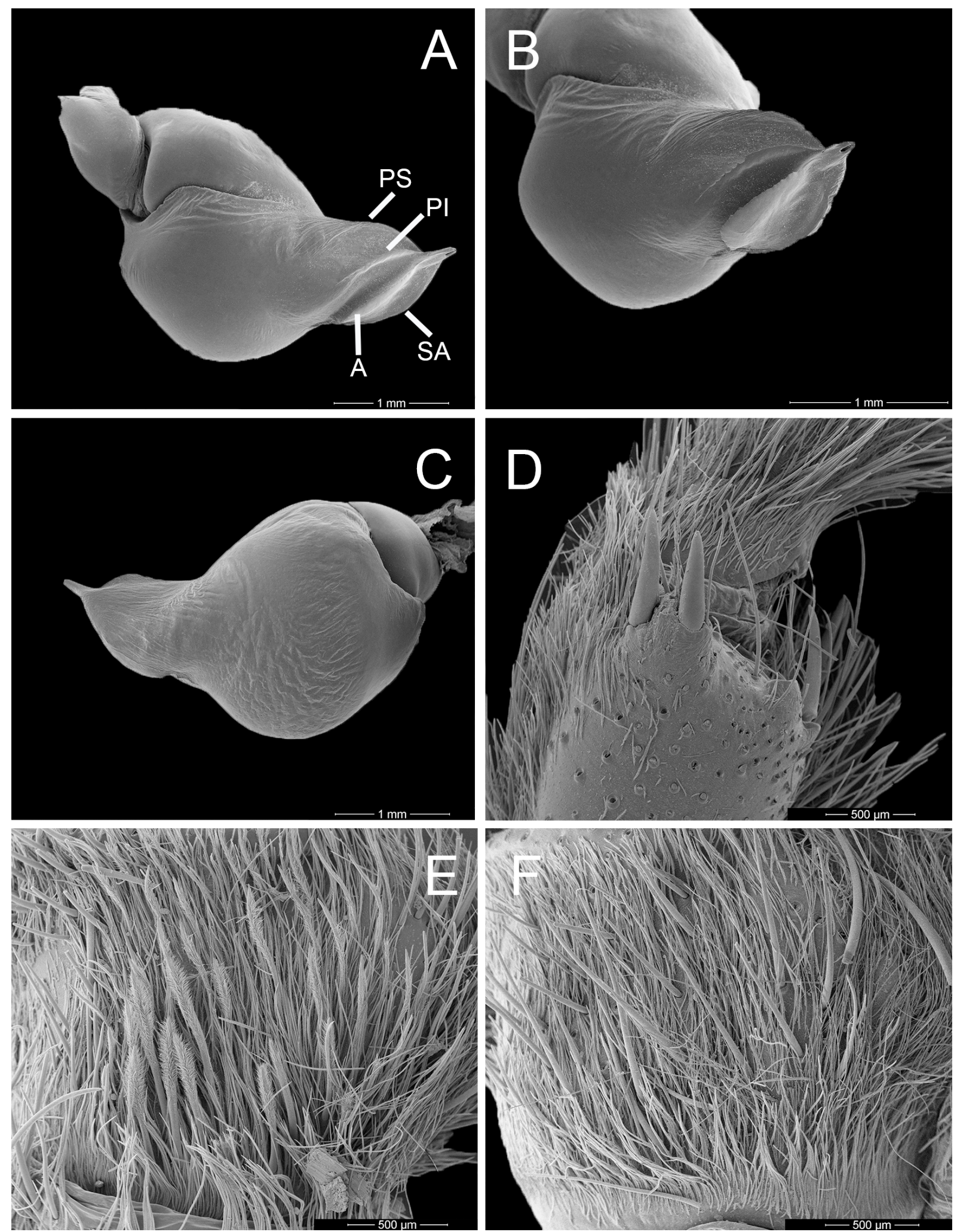

Fig. 17. Umbyquyra cuiaba gen. et sp. nov., holotype, Õ, Cuiabá, Mato Grosso, Brazil (IBSP 167423). A. Palp, prolateral. B. Anterolateral. C. Retrolateral. D. Tibial apophysis, retrolateral. E. Stridulatory organ, palp trochanter. F. Trochanter I. Abbreviations: see Material and methods. 
(UFMT 0974); 1 O, same collection data as for preceding, Oct. 1972, G.R. Kloss and F. Val leg. (MZSP 28890); 1 ô, same collection data as for preceding, Nov. 2006 (UFMT 0972).

\section{Additional material}

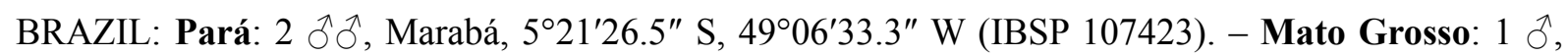
Cuiabá, $15^{\circ} 36^{\prime} 10.2^{\prime \prime} \mathrm{S}, 56^{\circ} 05^{\prime} 47.9^{\prime \prime}$ W, 8 Oct. 1989, M. Serrano leg. (IBSP 167428); 1 ðै, same collection data as for preceding, 8 Sep. 2000, M. Shiraiwa leg. (UFMT 0969); 1 \&, Barra do Garças, $15^{\circ} 53^{\prime 2} 26.7^{\prime \prime}$ S, $52^{\circ} 15^{\prime} 43.1^{\prime \prime}$ W, Feb. 1990, F.T. Carvalho leg. (IBSP 107159); 1 q, Parque Nacional da Chapada dos Guimarães, near Cachoeira Véu de Noiva, $15^{\circ} 24^{\prime} 22.9^{\prime \prime}$ S, 55 50 $50^{\prime} 1.1^{\prime \prime} \mathrm{W}, 11$ Aug. 2015, R.P. Indicatti and

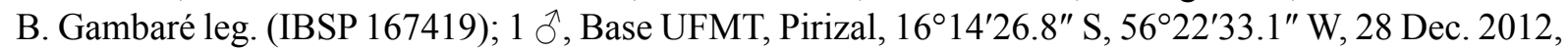

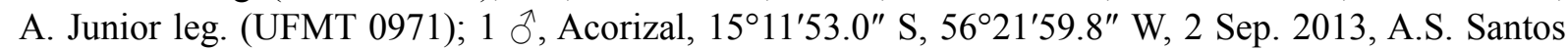

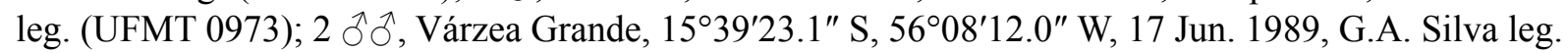
(IBSP 107159).

\section{Description}

Male (holotype, IBSP 10728)

In life: light brown carapace and legs. Abdomen dark brown covered by red hairs with dark patch on dorsal side (Fig. 16F). In ethanol: same color as U. paranaiba gen. et sp. nov. Total length: 33.0. Carapace: 13.5 long, 12.5 wide. Clypeus: 0.4. Eye tubercle: 1.6 long, 2.1 wide. Eyes: anterior row slightly procurved and posterior recurved (Fig. 16E). AME: 0.3 long, 0.3 wide; PME: 0.25 long, 0.17 wide; ALE: 0.2 long, 0.35 wide; PLE: 0.2 long, 0.35 wide. Basal segment of chelicerae with 11 teeth. Labium: 2.0 long, 2.0 wide, with 112 cuspules. Endites with 150-155 cuspules. Stridulatory bristles with around 15 bristles on palp trochanter and 45 on leg I (Fig. 17E-F). Palp: femur 7, patella 2.6, tibia 7.4, tarsus 2.7, total 20. Leg I femur 10.8, patella 4.5, tibia 9.4, metatarsus 10.3, tarsus 6.7, total 41.7 . II 12.7, 2.5, 8.5, 10.1, 6.6, 40.4. III 10.5, 2.0, 8.4, 9.7, 5.7, 36.3. IV 11.4, 3.0, 9.6, 11.2, 5.8, 50. Spination: palp: femur d0-0-2app, tibia v0-1p-0ap, p1-2-1-1-1-1-2-1-1-3ap, r0-1-3ap. Legs I tibia v2-1-1r-1-1r-11ap-1 apr-1app, r0-1-3ap, metatarsus v-1-0-0-1ap. II tibia v1-1r-1-1-1ap, p0-1-0-1-0ap, metatarsus v01-1-0-0-1ap, p0-1-0ap. III femur d0-0-1apr, tibia d0-0-1r-1apr, v1-1-0-1apr-2app, p0-1-1ap, r1-1-0-1ap, metatarsus d0-1p-1r-1p-0-0ap, v1-1p-1-1p-0-1apr-1ap-1 app, p0-1-0-0ap, r1-1-1-1ap. IV tibia v1-1-1-21r-1p-1-0ap, p0-1-1-0ap, r0-1-0-1-0-1ap, metatarsus d0-1r-1p-0-1ap, v1-1-1p-1r-1p-1r-2app-2apr, p00-1-0ap, r0-1-0-0-1 ap. Metatarsus I with a slight basal curvature. Tibial apophysis of leg I: retrolateral branch projected with two strong distal spines with 2-3 median setae; prolateral branch very short with a distal spine and a sub basal spine (Figs 16C, 17D). PLS basal, median and apical segments 1.8, 1.8, 2.0 long, respectively.

Female (paratype, IBSP 8933)

In ethanol: same color as Umbyquyra paranaiba gen. et sp. nov., except black abdomen in the posterior half (Fig. 16F). Total length: 31 . Carapace 13.5 long, 11 wide. Clypeus 0.35 . Eye tubercle 1.2 long, 2.0 wide. AME 0.4 long, 0.35 wide; PME 0.25 long, 0.25 wide; ALE 0.25 long, 0.45 wide; PLE 0.25 long, 0.2 wide. Basal segment of chelicerae with 10 teeth. Labium 1.5 long, 2 wide, with 100 cuspules. Endites with $120-125$ cuspules. Stridulatory bristles as in male. Palp: femur 8.1, patella 1.7 tibia 4.2 , tarsus 5, total 19. Leg I femur 9.6, patella 3.0, tibia 8.3, metatarsus 5.6, tarsus 4.2, total

Fig. 18 (opposite page). A-B. Umbyquyra acuminata (Schmidt \& Tesmoingt in Schmidt, 2005) gen. et comb. nov. Live specimen from Santa Cruz de la Sierra, Bolivia (photos by Cristian Grismado). C-F. Umbyquyra cuiaba gen. et sp. nov. C. Live specimen from Parque Nacional Chapada dos Guimarães, Mato Grosso, Brazil, dorsal. D. Defensive display. E. Inside burrow. F. Detail of burrow interior without web. G-H. Umbyquyra paranaiba gen. et sp. nov. G. Live specimen from São Paulo, São Paulo, Brazil, dorsal. H. Defensive display, frontal (C-H, photos by Rafael Indicatti). 
GARGIULO F. de F. et al., Umbyquyra gen. nov., new genus of Neotropical Theraphosidae
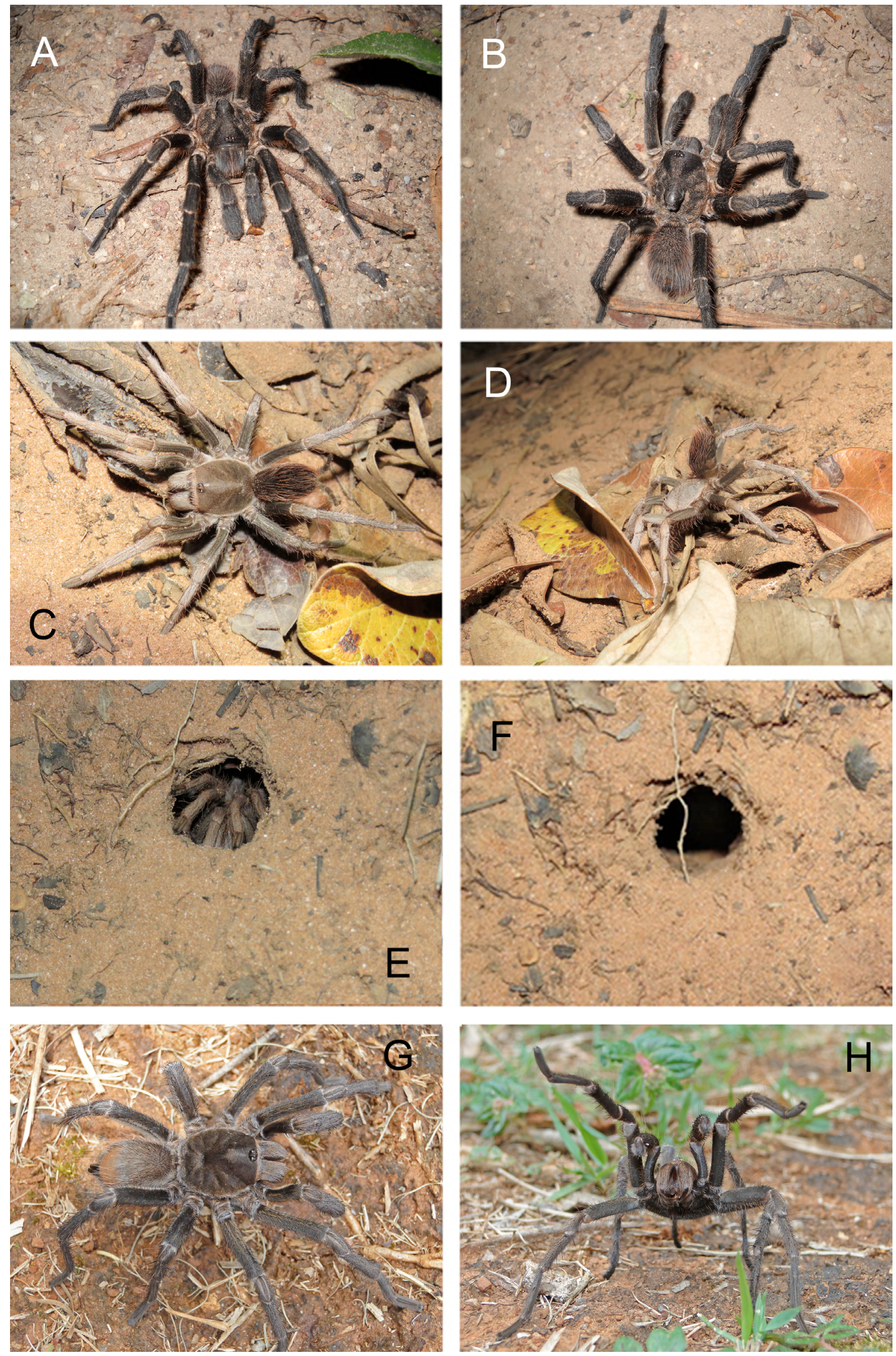
30.7. II femur 6.8, patella 3.5 , tibia 6.1 , metatarsus 4.8 , tarsus 3.8 , total 25 . III femur 6.8 , patella 4.0 , tibia 4.7, metatarsus 6.7, tarsus 4.0, total 26.2. IV femur 7.0, patella 4.0, tibia 10, metatarsus 10.5, tarsus 4.2, total 35.7. Spination: palp: femur d0-0-2app, tibia v0-1p-0ap, p1-2-1-1-1-1-2-1-1-3ap, r01-3ap. Leg I tibia v2-1-1r-1-1r-1-1ap-1apr-1app, r0-1-3ap, metatarsus v-1-0-0-1 ap. II tibia v1-1r-1-11ap, p0-1-0-1-0ap, metatarsus v0-1-1-0-0-1 ap, p0-1-0ap. III femur d0-0-1 apr, tibia d0-0-1r-1apr, v11-0-1apr-2app, p0-1-1ap, r1-1-0-1ap, metatarsus d0-1p-1r-1p-0-0ap, v1-1p-1-1p-0-1apr-1ap-1app, p0-1-0-0ap, r1-1-1-1 ap. IV tibia v1-1-1-2-1r-1p-1-0ap, p0-1-1-0ap, r0-1-0-1-0-1ap, metatarsus d01r-1 p-0-1 ap, v1-1-1p-1r-1 p-1r-2app-2apr, p0-0-1-0ap, r0-1-0-0-1ap. Metatarsus I with a slight basal curvature. PS basal, median and apical segments 1.8, 1.8, 2.0 long, respectively. Spermathecae with oval and elongated RS, with short ducts, apart from each other by approximately three times their diameter (Fig. 16D).

\section{Distribution}

Brazil: states of Mato Grosso and Pará (Fig. 31).

\section{Natural history}

This species is very aggressive, raising the abdomen or legs I when disturbed (Fig. 18D, H). The female from Parque Nacional da Chapada dos Guimarães (IBSP 167419) was collected in a dirt hill, inside a hole with a $3 \mathrm{~cm}$ opening diameter which did not contain any silk.

Umbyquyra araguaia gen. et sp. nov. urn:lsid:zoobank.org:act:E0F14424-01E1-4C65-8C76-BA55DC150B86

Figs 19-20, 30

\section{Diagnosis}

Males of Umbyquyra araguaia gen. et sp. nov. differ from those of the other species by an inconspicuous tibial apophysis (Figs 19C, 20D) and a retrolateral branch with two strong spines, and by a straight fovea without any projections on the carapace (Fig. 5E).

\section{Etymology}

The species epithet is a noun in apposition taken from the type locality.

\section{Type material}

Holotype

BRAZIL: Ō, Pará, Conceição do Araguaia, $8^{\circ} 15^{\prime} 10.8^{\prime \prime}$ S, 49¹9'11.7" W, Jun. 1979, W. Lourenço leg. (IBSP 112531).

\section{Paratype}

BRAZIL: Tocantins, $1{ }^{\AA}$, Palmas, Serra do Lajeado, $10^{\circ} 06^{\prime} 08.6^{\prime \prime} \mathrm{S}, 48^{\circ} 14^{\prime} 35.8^{\prime \prime} \mathrm{W}, 20$ Nov. 2002, J. Nunes leg. (IBSP 110473).

\section{Description}

Male (holotype, IBSP 110473)

In ethanol: same color as $U$. paranaiba gen. et sp. nov. Total length 18. Carapace 10.1 long, 8.3 wide. Clypeus 0.1. Eye tubercle 1.0 long, 1.6 wide. AME 0.25 long, 0.27 wide; PME 0.25 long, 0.2 wide; ALE 0.3 long, 0.2 wide; PLE 0.3 long, 0.2 wide. Basal segment of chelicerae with 11 teeth. Labium 1.3 long, 2.2 wide, with 103 cuspules. Endites with 122-124 cuspules. Stridulatory bristles with around 15 bristles on palp trochanter and 45 on leg I (Fig. 19E-F). Palp: femur 3.8, patella 2.7, tibia 4.5, tarsus 2.1, total 13.1. Leg I femur 6.7, patella 5.0, tibia 8.6, metatarsus 6.7, tarsus 4.7, total 
31.7. II 6.8, 4.2, 7, 7, 3, 28. III 5.8, 3.5, 9, 11.5, 5.2, 35. IV 6.6, 3.6, 9.2 10.4, 3.7, 33.5. Spination: palp: femur tibia d0-0-1apr. Leg I tibia p0-1-0-1-2ap, metatarsus v1-0-1-1-0-1ap. II femur, 0-0-01 app, tibia v1-2-1 ap-2app, p0-1-0-1-0ap, metatarsus d0-0-1p-0ap, v0-1-1-0-1 apr-1ap-1 app, p0-1-0ap. III tibia v0-0-0-1-1r-1 p-0-1-0-2app-1 apr, p0-0-1-0-0ap, r0-1-0-1ap, metatarsus v0-1-1p-1r-0-1-1r-1p1-1p-2-2ap, p0-0-1-1-0-1 ap, r0-1-0-0ap. IV tibia v0-0-1-1 p-1r-1-2apr, 1-1-1ap, r0-1-0ap, metatarsus d1 r-0-1r-1 p-0-1 app-1apr, v1-1-1-1-2-1-1 app-apr-1ap, p0-1-0-1ap, r0-1-0-1ap. Tibial apophysis of leg I: prolateral branch absent and two strong distal spines on retrolateral branch (Figs 19C, 20D). Palpal bulb with semicircular projected A and long SA (Figs 19A, 20A). PLS basal, median and apical segments 1.4, 1.4, 2.3 long, respectively.

\section{Female}

Unknown.

\section{Distribution}

Brazil: states of Pará and Tocantins (Fig. 30).
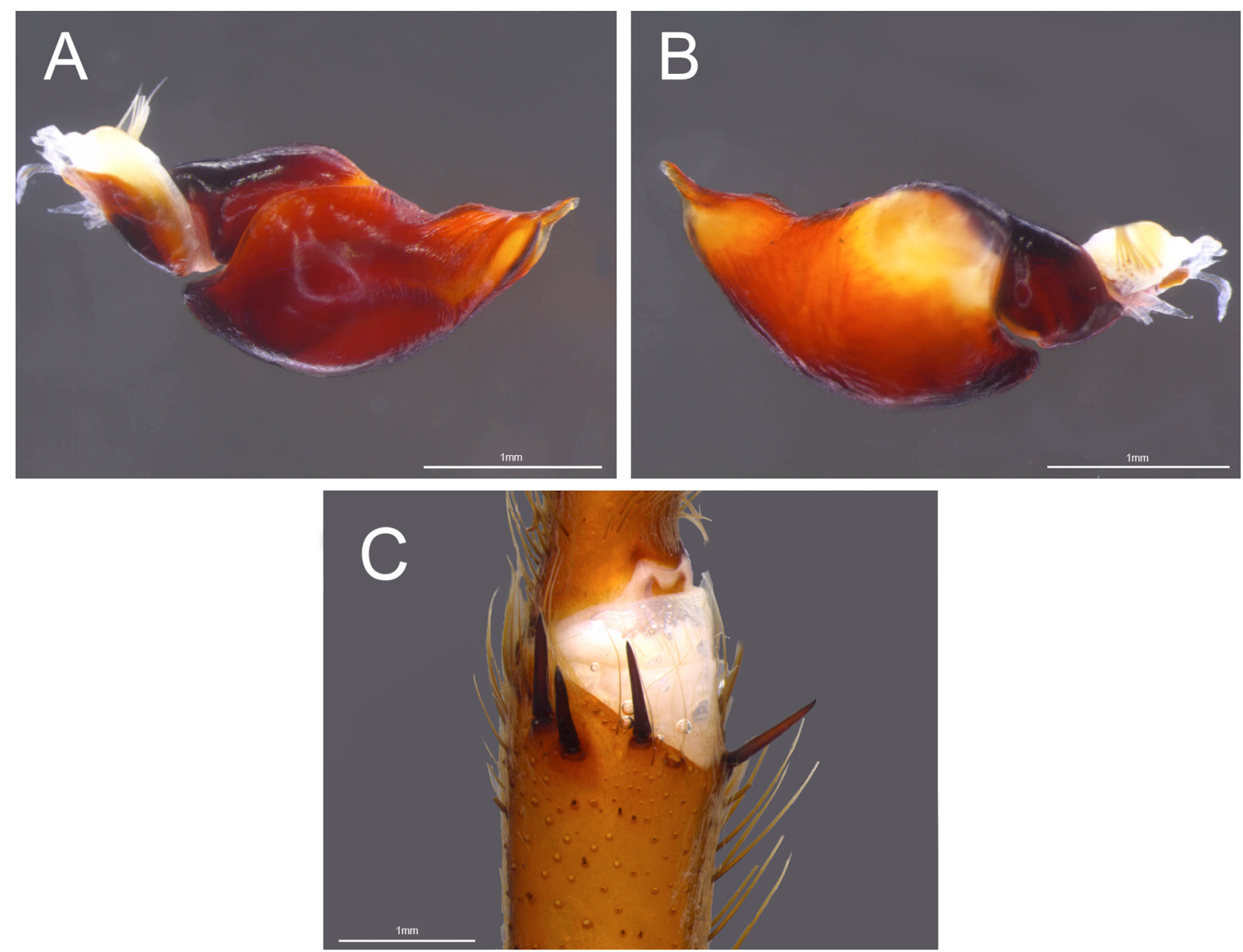

Fig. 19. Umbyquyra araguaia gen. et sp. nov., paratype, $\widehat{\partial}$, Serra do Lajeado, Tocantins, Brazil (IBSP 110473). A. Palp, prolateral. B. Retrolateral. C. Tibial apophysis, retrolateral. 

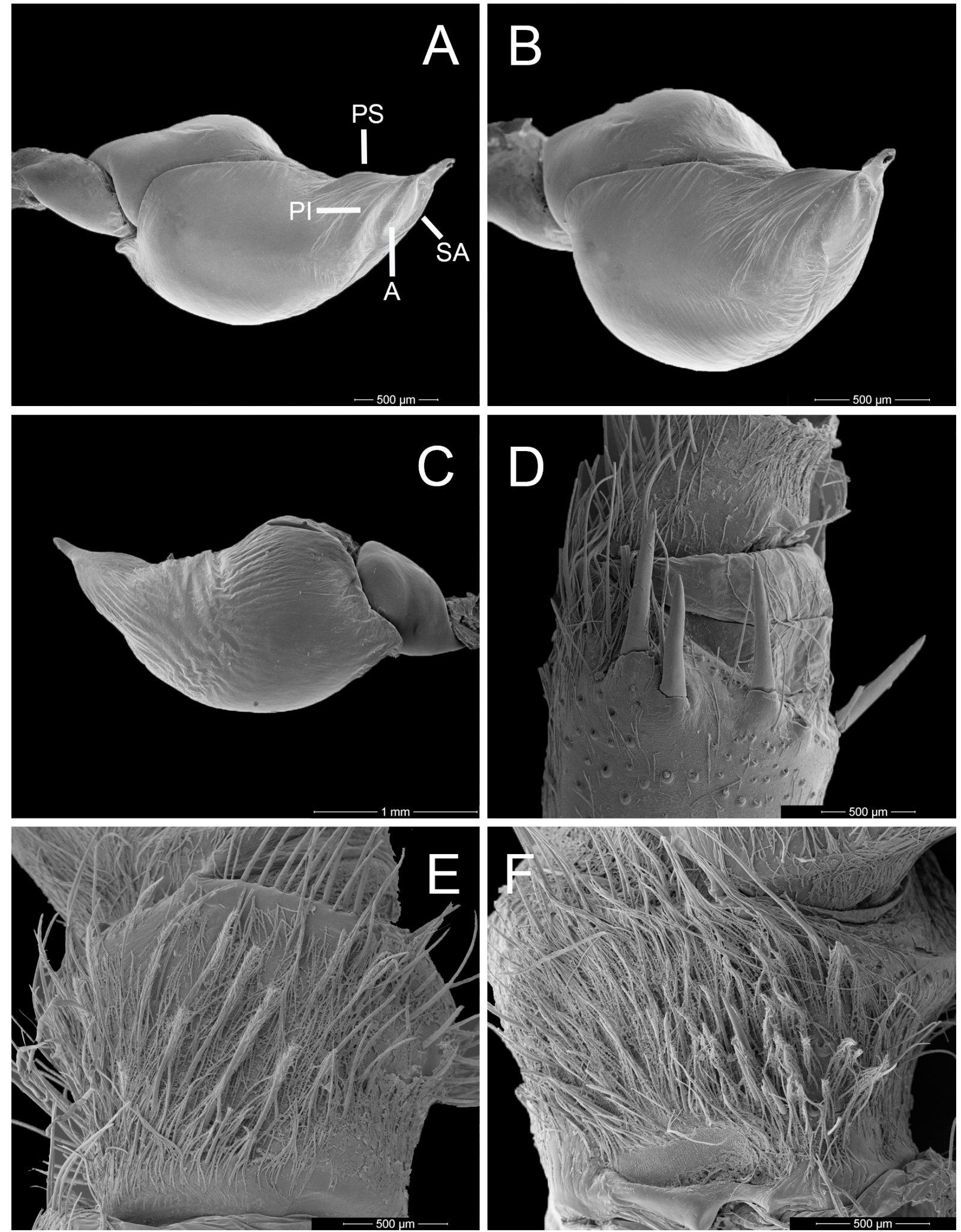

Fig. 20. Umbyquyra araguaia gen. et sp. nov., paratype, Ô, Serra do Lajeado, Tocantins, Brazil (IBSP 110473). A. Palp, prolateral. B. Anterolateral. C. Retrolateral. D. Tibial apophysis, retrolateral. E. Stridulatory organ, palp trochanter. F. Trochanter I. Abbreviations: see Material and methods. 
Umbyquyra belterra gen. et sp. nov.

urn:1sid:zoobank.org:act:E6C30EB4-D131-4060-8B26-F5394BE3AAD9

Figs 21-22, 29

\section{Diagnosis}

Males and females of Umbyquyra belterra gen. et sp. nov. differ from those of all other species by a recurved fovea (Fig. 5F) and a very long prolateral inferior keel of the male palpal bulb which extends for more than half of the prolateral face of the tegulum (Fig. 22C). This species presents five keels, including the PAc keel near the PS keel. This is a character shared only between two species of Umbyquyra gen. nov. Females differ from those of the other species by a spermathecae with SR curved sideways, with long ducts and apart from each other by more than five times their diameter (Fig. 21D).

\section{Etymology}

The species epithet is a noun in apposition taken from the type locality.
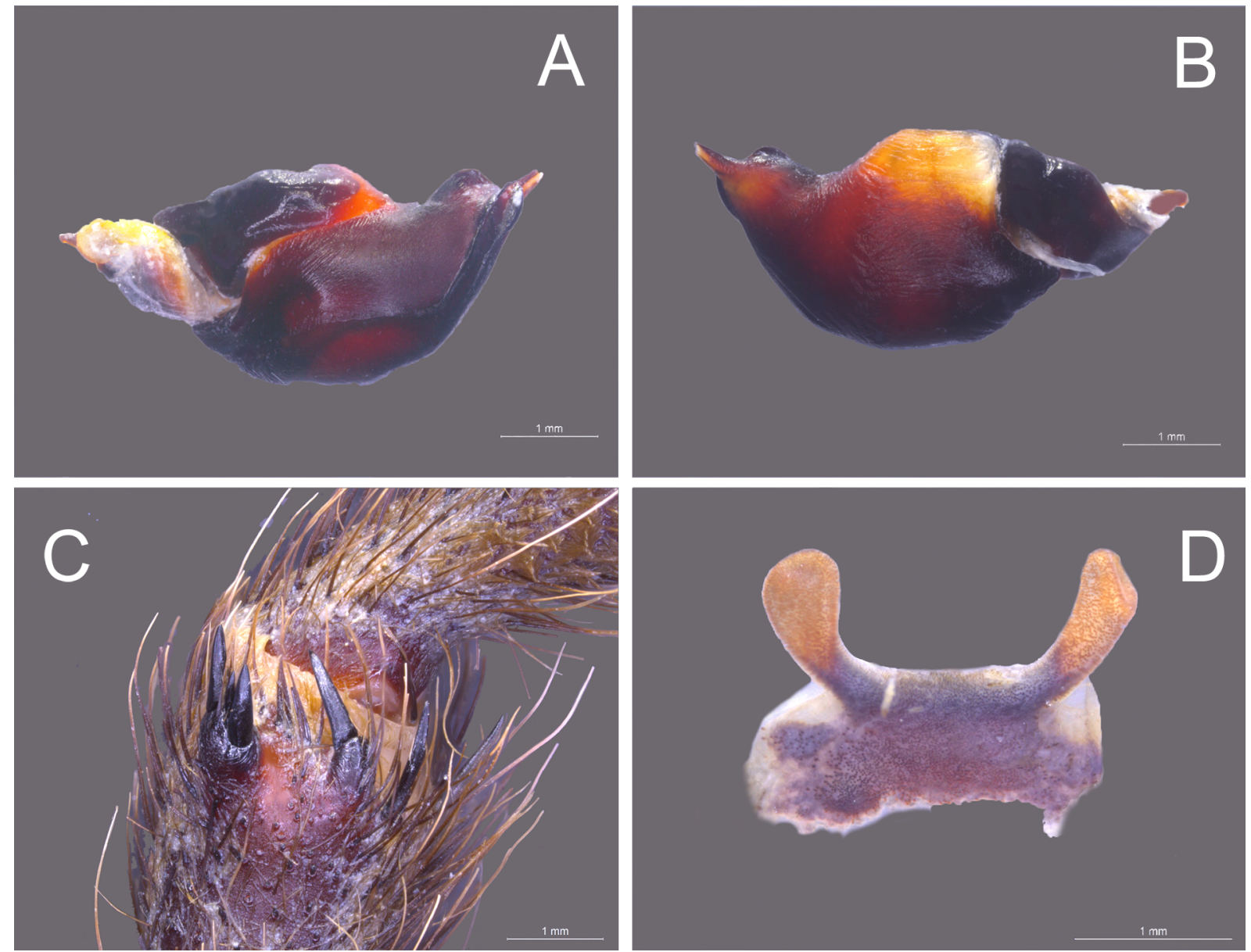

Fig. 21. Umbyquyra belterra gen. et sp. nov. A-C. Holotype, ô, Flona do Tapajós, Belterra, Pará, Brazil (IBSP 167421). A. Palp, prolateral. B. Retrolateral. C. Tibial apophysis, retrolateral. D. Paratype, ô, spermathecae, dorsal (IBSP 167420). 


\section{Type material}

Holotype

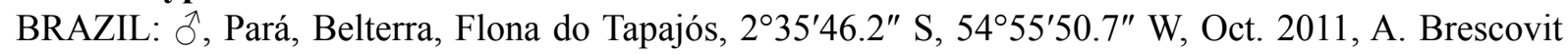
et al. leg. (IBSP 167421).

\section{Paratypes}

BRAZIL: Pará, 1 q, Belterra, Flona do Tapajós, 2³5'46.2" S, 5455'50.7" W, Oct. 2011, A.D. Brescovit et al. leg. (IBSP 167420); 6 $\lesssim \widehat{o}$ (IBSP 167422, IBSP 170011, IBSP 170012, FIT/UNAMA 1195, FIT/ UNAMA 615, FIT/UNAMA 616), 2 + + (FIT/UNAMA 486, FIT/UNAMA 482), same data as for preceding.

\section{Description}

Male (holotype, IBSP 167421)

In ethanol: carapace dark-brown as dorsal face of long, 0.5 abdomen. Total length 41. Carapace 19.4 long, 16.3 wide. Clypeus 0.3. Eye tubercle 1.8 long, 2.7 wide. AME 0.5 wide; PME 0.3 long, 0.2 wide; ALE 0.3 long, 0.6 wide; PLE 0.3 long, 0.4 wide. Basal segment of chelicerae with 10 teeth. Labium: 2.2 long, 3.1 wide, with 128 cuspules. Endites with 165-168 cuspules. Stridulatory organ contains more than 10 very evident bristles on palp trochanter and leg I (Fig. 22E-F). Palp: femur 9.8, patella 4.2, tibia 9, tarsus 2.8 , total 25.8. Tarsi II very short. Leg I femur 18.4, patella 4.7, tibia 16, metatarsus 14.3, tarsus 8.3, total 61.7. II 15.5, 3.5, 14.5, 14, 5.4, 52.9. III 16, 5.7, 12.2, 15.3, 7.8, 57. IV 18.2, 8, 16, 22.9, 9, 74. Spination: palp: tibia v0-0-1r0ap, p1-1-1-1-1-1-1-1-1ap, r0-0-1-0ap. Legs I femur d0-0-1app, tibia v2-0-2-0-2ap, p0-1-0-1-0ap, metatarsus v0-1-0-0-0-1apr-1-1app. II tibia v2-0-2-1r-1r-1app-1apr, p0-1-0-1-0ap, metatarsus v0-1-1-0-1app-1apr, p0-1-0-1ap. III femur d0-0-1apr, tibia v1-1p-1p-1-01app-1 apr, p0-1-0-1-0ap, r1-1-1-1 app, metatarsus d0-0-0-1 app-1 apr, v1-1 p-1p-1r-1-1p-1 p-1 p-1r-1 app1-1 apr, p1-0-1-1-0-1-1 ap, r0-0-1-0-0-1 ap. IV tibia v1-1-1r-1p-1-1p-1-1 apr, p0-1-1-0-2ap, r1-0-0-1-1ap, metatarsus v1-1-1-1p-1r-1-1p-1-1r-1-1-1-1p-1-1r-1p-1-1-1 app-1ap-1apr, p0-0-1-0-1ap, r0-0-1-0-0-10ap. Metatarsus I with a slight basal curvature. Tibial apophysis of leg I: retrolateral branch projected with two strong distal spines with two median setae; prolateral branch projected with a distal spine and sub basal one (Figs 21C, 22D). PLS basal, median and apical segments 3.1, 2.6, 3.2 long, respectively. Male palp with PAc (Fig. 22A).

Female (paratype, IBSP 167420)

In ethanol, same color as male. Total length (damaged). Carapace (damaged). Clypeus: 0.2. AME: 0.5 long, 0.5 wide; PME 0.4 long, 0.3 wide; ALE: 0.4 long, 0.2 wide; PLE: 0.4 long, 0.3 wide. Basal segment of chelicerae with 11 teeth. Labium: 2.3 long, 3 wide. Eye tubercle 2.8 long, 2.1 wide. Stridulatory bristles as in male. Palp: femur 11.2, patella 4.5, tibia 9.8, tarsus 7.5, total 33. Tarsi II short as in male. Leg I femur 15.5, patella 9, tibia 14.2, metatarsus 11, tarsus 7.4, total 57.2. II 12.6, 8.1, 12.6, 11, 7.2, 51.5. III 13, 7.7, 12, 11, 7.1, 50.8. IV absent. Spination: palp: femur p0-1-0ap. Legs I tibia v0-0-1p1r-1app-1ap-2apr, metatarsus d0-1 p-0ap, v-0-1-2ap. II tibia v0-1-1app-1ap-1apr, p-0-1-0ap, metatarsus v01-1-0-1 app-1apr. III tibia v0-1-0-1-0-1p-1p-1apr, p0-1-0ap, metatarsus v0-1-1-0-1apr-1ap-1app, p01-0ap. IV tibia d1r-1r-1apr, v0-1-1p-1ap-2app, p0-1-0-1-0ap, r0-1-0-1-0-1ap, metatarsus d1p-1r-1app, v2r-1 p-1r-1p-1r-1apr-1ap, p0-1-0ap, r0-1-0ap. Spermathecae with two RS with long ducts and more or less pentagonal basal area (Fig. 21D).

\section{Remarks}

The female is in bad condition, so total length and carapace could not be measured.

\section{Distribution}

Brazil: state of Pará (Fig. 29). 

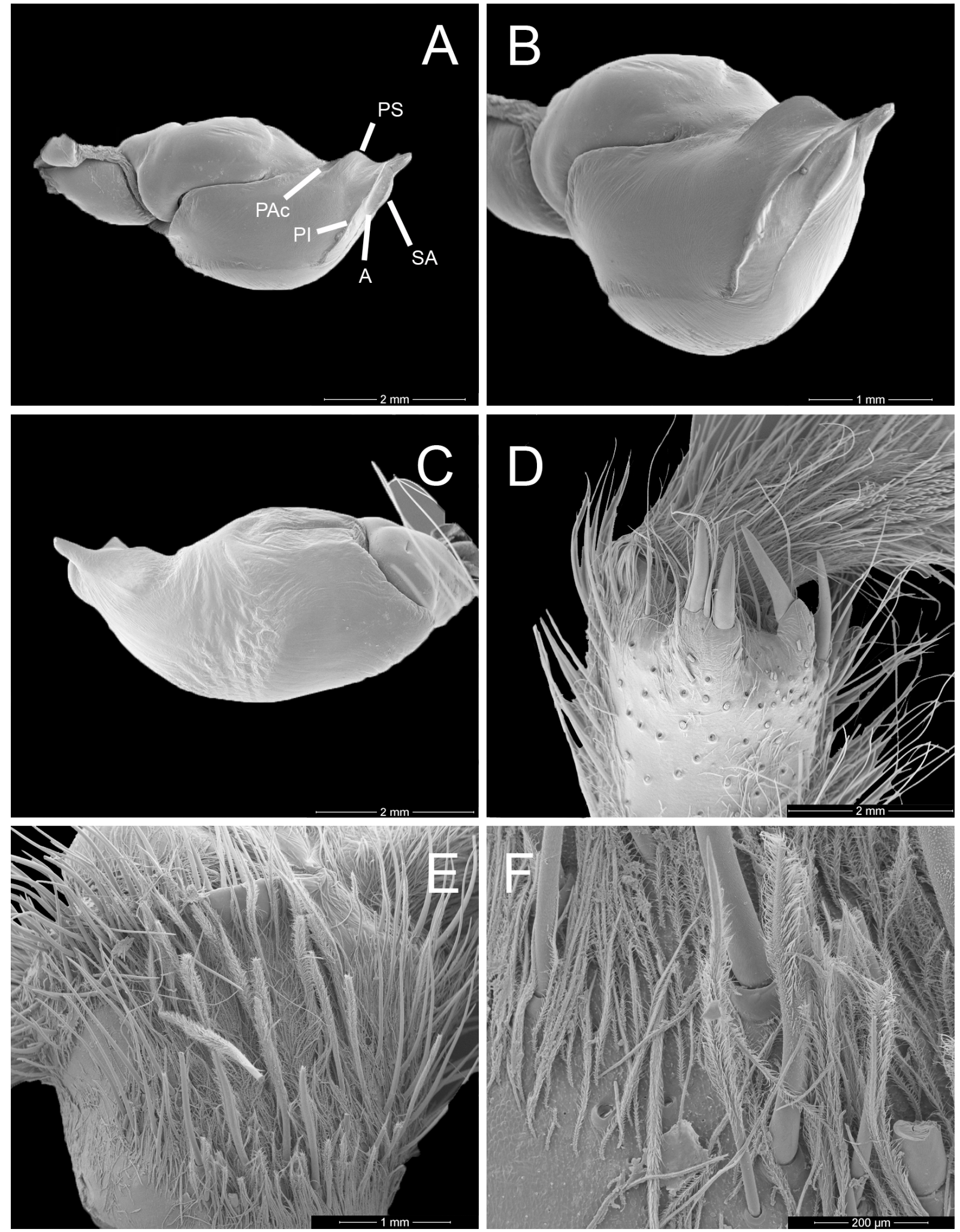

Fig. 22. Umbyquyra belterra gen. et sp. nov., holotype, ô, Flona do Tapajós, Belterra, Pará, Brazil (IBSP 167421). A. Palp, prolateral. B. Anterolateral. C. Retrolateral. D. Tibial apophysis, retrolateral. E. Stridulatory organ, trochanter. F. Trochanter I. Abbreviations: see Material and methods. 
Umbyquyra tapajos gen. et sp. nov.

urn:1sid:zoobank.org:act:C862CAB2-4585-4B9C-8364-3DB8978445E7

Figs 23-24, 29

\section{Diagnosis}

Males of Umbyquyra tapajos gen. et sp. nov. resemble those of $U$. belterra gen. et sp. nov. by the presence of a PAc (Fig. 23C), but differ by a more compact tegulum and keels being parallel to each other (Figs 23A-C, 24A-C). Umbyquyra tapajos gen. et sp. nov. presents a short and straight fovea while $U$. belterra have a recurve fovea.

\section{Etymology}

The species epithet is a noun in apposition taken from the type locality.

\section{Type material}

\section{Holotype}

BRAZIL: O, Pará, Belterra, Flona do Tapajós, 2³5'46.2" S, 5455'50.7" W, Oct. 2011, A.D. Brescovit et al. leg. (IBSP 161738).
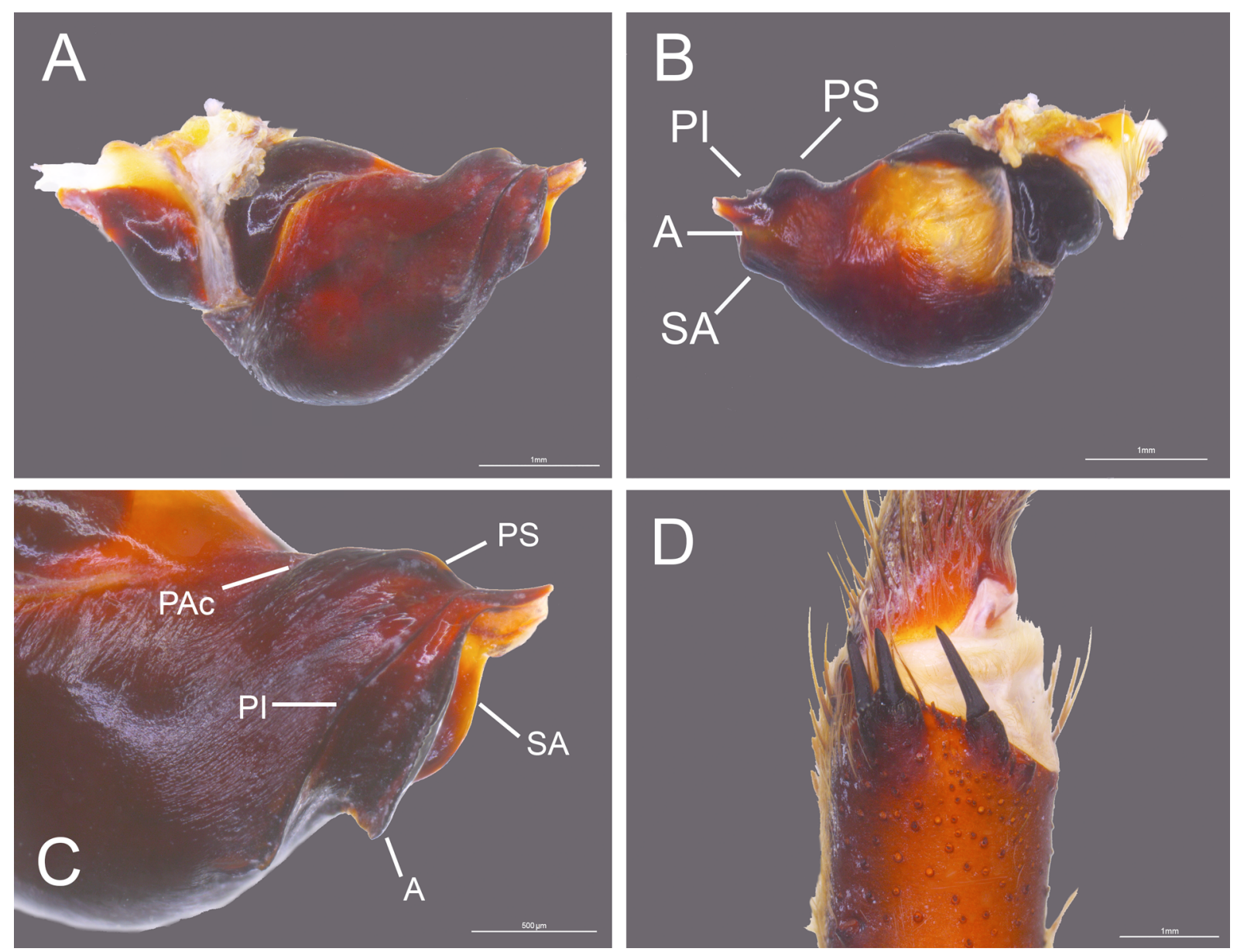

Fig. 23. Umbyquyra tapajos gen. et sp. nov., paratype, đ̂, Flona do Tapajós, Belterra, Pará, Brazil (IBSP 161728). A. Palp, prolateral. B. Retrolateral. C. Anterolateral, distal area. D. Tibial apophysis, retrolateral. Abbreviations: see Material and methods. 

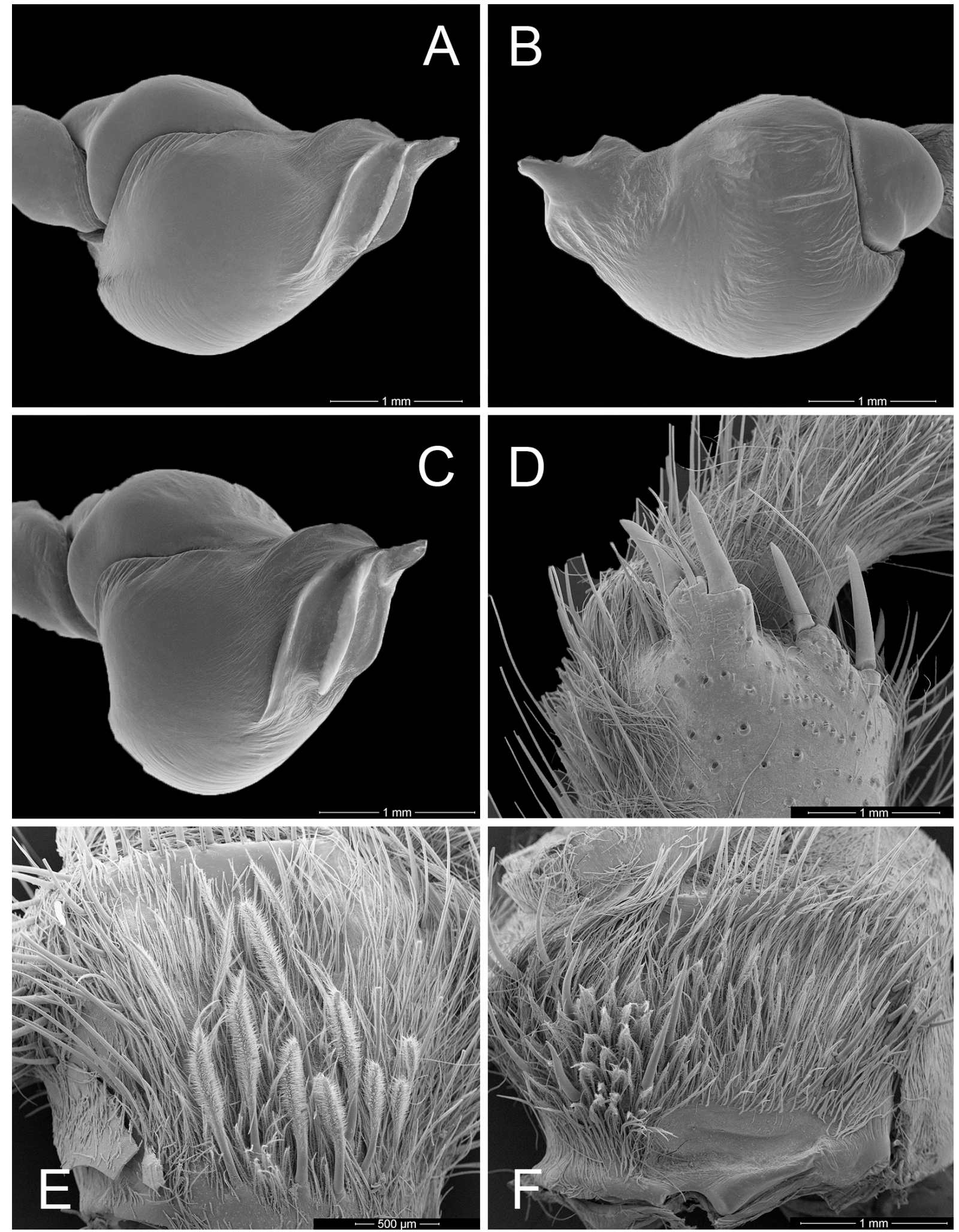

Fig. 24. Umbyquyra tapajos gen. et sp. nov., paratype, đ̂, Flona do Tapajós, Belterra, Pará, Brazil (IBSP 161728). A. Palp, prolateral. B. Retrolateral. C. Anterolateral. D. Tibial apophysis, retrolateral. E. Stridulatory organ, trochanter. F. Trochanter I. 
Paratype

BRAZIL, 1 §ै, same data as for holotype (IBSP 161728).

\section{Description}

Male (holotype, IBSP 161738)

In ethanol: same color as $U$. belterra gen. et sp. nov. Total length 32. Carapace 12.3 long, 16.4 wide. Clypeus 0.6. Eye tubercle 2.3 long, 1.6 wide. Eyes: anterior row slightly procurved and posterior recurved. AME 0.41 long, 0.45 wide; PME 0.2 long, 0.3 wide; ALE 0.27 long, 0.53 wide; PLE 0.25 long, 0.44 wide. Basal segment of chelicerae with 12 teeth. Labium: 2.2 long, 1.7 wide, with 120 cuspules. Endites with 180-183 cuspules. Stridulatory bristles with around 10-12 bristles on palp trochanter and 35-45 on leg I (Fig. 24E-F). Palp: femur 7.7, patella 3.2, tibia 7.6, tarsus 2.7, total 21.2. Leg I femur 14.3, patella 4.8, tibia 12.5, metatarsus 12.6 , tarsus 7.5 , total 51.7. II 13.8, 4.1, 9.7, 12.5, 6.4, 46.5. III 13.0, 4.6, 9.7, 14.0, 7.0, 48.3. IV 14.7, 4.2, 13.3, 18.7, 7.1, 58. Spination: palp: femur d0-0-1app, tibia v0-1r-0ap, p11-2-1-1-1-2-1-2ap, r0-0-1-0ap. Legs I femur d0-0-1app, tibia v3-1-1-1-1apr-1app, p0-1-0-0-0ap, p0-10-0-0ap, metatarsus v0-0-0-1 app-1 apr, p0-1-0-0ap, r0-1-0-0ap. II femur, 0-0-0-1app, tibia v0-0-1-1-0ap, p0-1-0-1-0ap, r1-0-1-1-0ap, metatarsus v1-2-1-1 p-1r-0-1app, p1-0-1-0-0ap, r0-1-0-0-1ap. III femur d00-1apr, patella r0-1-0ap, tibia v0-0-1-1-0ap, 0-1-0-1-0ap, r1-0-1-1-0ap, metatarsus v1-2-1-1p-1r-0-1app, p1-0-1-0-0ap, r0-1-0-0-1ap. IV femur d0-0-1apr, tibia v0-1p-1-1p-1r-1p-1app-1ap-1apr, p1-1-1-1-1ap, metatarsus v1-1r-1p-1-1-1p-2-1p-1r-1app-1ap-1apr, p1-0-1-0-1-0ap, r0-1-0-0-1-0ap. Tibial apophysis of leg I: retrolateral branch projected with two strong distal spines with two median setae; prolateral branch slightly projected with distal and sub basal spines (Figs 23D, 24D). PLS basal, median and apical segments $2.5,2.3,3.0$ long, respectively.

\section{Female}

Unknown.

\section{Distribution}

Brazil: state of Pará (Fig. 29).

$$
\begin{gathered}
\text { Umbyquyra tucurui gen. et sp. nov. } \\
\text { urn:1sid:zoobank.org:act:B9C76D3F-B8D8-4DA1-B47C-315D34E05706 }
\end{gathered}
$$

Figs 6, 25-26, 29

\section{Diagnosis}

Males and females of Umbyquyra tucurui gen. et sp. nov. resemble those of $U$. paranaiba gen. et sp. nov. by a straight fovea (Fig. 5E), but this species can be distinguished from U. paranaiba gen. et sp. nov. and the other species by a retrolateral branch of the tibial apophysis with 5-6 strong distal spines (Figs 25C, 26D), and prolateral inferior and accessory keels being parallel to each other (Figs 25A, 26A-B). The female spermathecae resemble those of Umbyquyra paranaiba gen. et sp. nov., but in U. tucurui gen. et sp. nov. the base is narrower (Fig. 25D).

\section{Etymology}

The species epithet is a noun in apposition taken from the type locality.

\section{Type material}

\section{Holotype}

BRAZIL: Ô, Pará, Tucuruí, 346'02.9" S, 4941'10.2" W, 17 Nov. 1987, S.M. Lucas leg. (IBSP 104834). 
GARGIULO F. de F. et al., Umbyquyra gen. nov., new genus of Neotropical Theraphosidae

\section{Paratype}

BRAZIL: Pará, 1 ㅇ, Tucuruí, 346'02.9" S, 4941'10.2" W, Equipe Resgate de Fauna leg. (IBSP 107431).

\section{Additional material examined}

BRAZIL: Pará: 1 O̊, Tucuruí, Usina Hidrelétrica de Tucuruí, 349'58.3" S, 49³9’02.0" W, 25 Jun. 1987 (IBSP 111694); $1 \delta^{\top}$, Ilha do Bananal, $3^{\circ} 17^{\prime} 45.4^{\prime \prime} \mathrm{S}, 49^{\circ} 39^{\prime} 26.4^{\prime \prime} \mathrm{W}$, (IBSP 107465); 1 q, same collection data as for preceding, 1984 (IBSP 113965); 1 o , Ilha de Tocantins, $3^{\circ} 17^{\prime} 36.1^{\prime \prime} \mathrm{S}, 49^{\circ} 39^{\prime 2} 20.7^{\prime \prime} \mathrm{W}$, Equipe Resgate de Fauna leg. (IBSP 107414); 1 juv., same collection data as for preceding (IBSP 107625); 1 $\widehat{\partial}$, same collection data as for preceding, 1984 (IBSP 107931); 1 ${ }^{\lambda}$, same collection data as for preceding (IBSP 107413); $1 \hat{\partial}$, same collection data as for preceding (IBSP 107707); $1 \hat{\partial}$, same collection data as for preceding (IBSP 107466); 1 q , same collection data as for preceding (IBSP 107417); $1 \hat{\jmath}$, same collection data as for preceding (IBSP 107419); 1 , , same collection data as for preceding (IBSP 107622); 1 , same collection data as for preceding (IBSP 107416); 1 ${ }^{\lambda}$, Vale do Caraipé, $3^{\circ} 37^{\prime} 57.3^{\prime \prime} \mathrm{S}, 4^{\circ} 38^{\prime} 56.6^{\prime \prime} \mathrm{W}$ (IBSP 107708); $2 \hat{\jmath}, 1$ 甲, Marabá, $5^{\circ} 22^{\prime} 47.7^{\prime \prime} \mathrm{S}, 4^{\circ} 07^{\prime} 57.4^{\prime \prime} \mathrm{W}$ (IBSP 102657), Equipe Resgate de Fauna leg.; $1 \hat{\jmath}$, same collection data as for preceding, 17 Nov. 1987 (IBSP 102834); $1 \hat{\partial}$, Brejo Grande

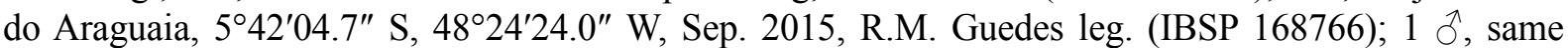
collection data as for preceding, Sep. 2015 (IBSP 168767); 1 \&, same collection data as for preceding, Sep. 2015 (IBSP 168764); 1 ڤ̆, same collection data as for preceding, Sep. 2015 (IBSP 168765).
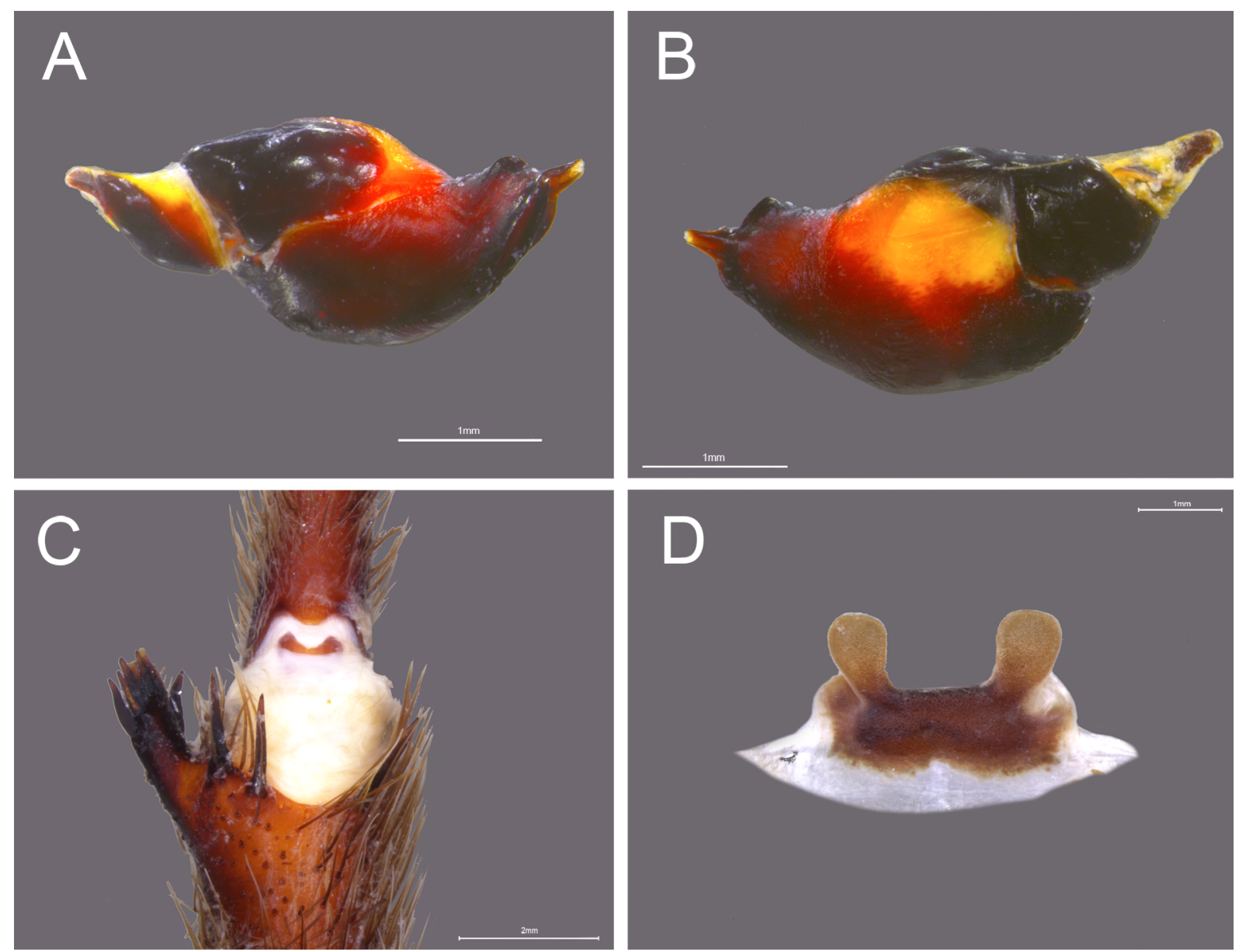

Fig. 25. Umbyquyra tucurui gen. et sp. nov. A-C. Holotype, đ̂, Tucuruí, Pará, Brazil (IBSP 104834). A. Palp, prolateral. B. Retrolateral. C. Tibial apophysis, ventral. D. Paratype, + , spermathecae, dorsal (IBSP 107431). 

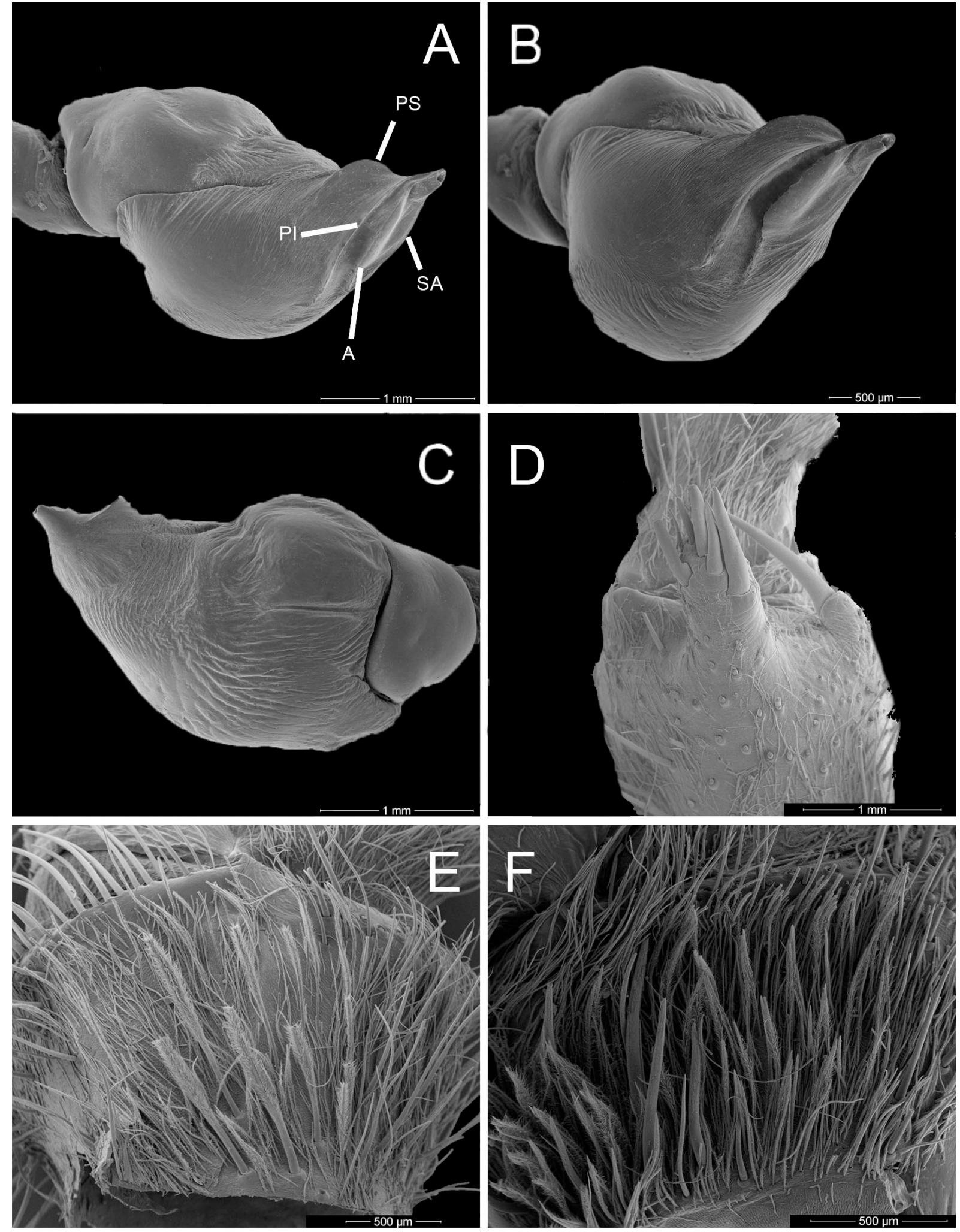

Fig. 26. Umbyquyra tucurui gen. et sp. nov., holotype, ô, Tucuruí, Pará, Brazil (IBSP 104834). A. Palp, prolateral. B. Anterolateral. C. Retrolateral. D. Tibial apophysis, retrolateral. E. Stridulatory organ, trochanter. F. Trochanter I. Abbreviations: see Material and methods. 


\section{Description}

Male (holotype, IBSP 104834)

In ethanol: color as $U$. belterra gen. et sp. nov. Total length 37. Carapace 13.5 long, 12 wide. Clypeus 0.2. Eye tubercle 1.3 long, 2 wide. AME 0.4 long, 0.4 wide; PME 0.3 long, 0.2 wide; ALE 0.3 long, 0.2 wide; PLE 0.4 long, 0.2 wide. Basal segment of chelicerae with 13 teeth. Labium 1.4 long, 1.8 wide, with 98 cuspules. Endites with 112-115 cuspules. Stridulatory organ with less than 10 long bristles on palp trochanter and on leg I (Fig. 26E-F). Palp: femur 6.3, patella 3.5, tibia 5.6, tarsus 2.7, total 18.1. Leg I femur 8.5, patella 6.5, tibia 10.5, metatarsus 9.5, tarsus 3.0, total 38. II 11.6, 6.4, 10.8, 9.5, 4.2, 42.5. III 10.1, 5.1, 8, 7, 5, 35.2. IV 12.7, 5.5, 11.3, 15.2, 6.1, 50.8. Spination: palp: patella d0-1p-1p-00ap, tibia d0-0-1-1-0-0ap, v0-0-0-1r-1r-1r-0-1apr, p0-0-2-1-2-1-1-1-2-2-1app-2ap, r0-0-0-1-1ap. Leg I femur d0-0-1-0app, tibia d0-1p-1p-1p-1p-0-0ap, v0-0-0-1r-1r-1r-0-1apr, p0-1-0ap, metatarsus v0-1-01app-1 ap-1 apr. II femur, 0-0-0-1 p-0ap, tibia d0-1 p-1p-0ap, v2r-0-2r-0-1r-1apr, metatarsus v0-1r-0-1r-01ap, p0-1-0-1 ap. III femur d0-0-1r-0ap, tibia v1-0-1-1 p-1r-0-2app-1 apr, p0-2-0-1-1-0-1ap, r1-0-1-0-1ap, metatarsus 0-0-0-1 app-1 apr, v0-1-1r-1p-1-1-1 p-1r-0-0-0-2ap-1 apr, p1-0-1-0-0ap, r0-1-0-0ap. IV femur r0-1-0-1-0-1ap, tibia p0-0-1-0ap, metatarsus d0-0-1-0-1-0ap, v1r-1-1p-1r-1p-1r-1r-1p-1r-1r-1p-1apr, p1-0-0-0-1-0-0-ap, r1-0-0-1-1-1-1p-0-1apr-1 ap-1app. Tibial apophysis of leg I: retrolateral branch strongly projected; prolateral branch slightly less projected with very long distal spine and shorter sub basal spine (Figs 25C, 26D). PLS basal, median and apical segments 1.5, 1.5, 2.4 long, respectively.

Female (paratype, IBSP 107431)

In ethanol: same color as male. Total length 44. Carapace 21 long, 16 wide. Clypeus 0.4. AME 0.4 long, 0.4 wide; PME 0.2 long, 0.2 wide; ALE 0.2 long, 0.5 wide; PLE 0.2 long, 0.4 wide. Basal segment of chelicerae with 10 teeth. Labium 2 long, 2.5 wide, with 98 cuspules. Endites with 111-108 cuspules. Eye tubercle 1.6 long, 2.8 wide. Stridulatory organ as in male. Palp: femur 7.8, patella 4.6, tibia 4.1, tarsus 4.2, total 20.7. Leg I femur 11, patella 8.2, tibia 6.3, metatarsus 3.8, tarsus 3.5, total 32.8. II 9.6, $6,6.6,7,3.8,33$. III 8.1, 5.8, 7, 8.3, 4.1, 33.3. IV 10.5, 6.1, 8.6, 11.6, 4.2, 41. Spination: palp: tibia v10-1r-1r-1apr, p0-1-0ap. Legs I femur d0-0-1p-0ap, tibia v0-1-0-0-1ap, metatarsus v0-1-0-0-0-1ap-1apr. II tibia v0-1-0-1ap, p0-1-1-0ap, metatarsus v0-1-0-1app-1apr, p0-1-0ap, r0-0-2ap. III tibia v0-0-1app1apr, p1-0-0ap, metatarsus d0-0-1r-0-0-1-0ap, v0-1-1p-1r-0-1r-1app, p1-1-1ap. IV tibia v-0-1p-1-1app1apr, p0-1-1 ap, metatarsus d0-0-1-0ap, v1r-1r-1p-1r-2r-1p-1r-1r-1apr. PLS basal, median and apical segments 2.1, 1.7, 3.1 long, respectively. Spermathecae with RS rounded, separated by approximately two diameters (Fig. 25D).

\section{Distribution}

Brazil: state of Pará (Fig. 29).

Umbyquyra caxiuana gen. et sp. nov. urn:1sid:zoobank.org:act:50CC5123-97AD-432D-AA63-A003BB696BE7

Figs 5C, 27-29

\section{Diagnosis}

Species of Umbuquyra caxiuana gen. et sp. nov. are distinguished from those of the other species by the large and procurve fovea (Fig. 5C), male palpal bulb with a shortened and compact tegulum, a superior keel very evident being parallel to prolateral accessory keels (Fig. 28B). The female can be distinguished by a spermathecae with globular SR, close to each other emerging from a common base forming a V shape (Fig. 27D).

\section{Etymology}

The species epithet is a noun in apposition taken from the type locality. 


\section{Type material}

\section{Holotype}

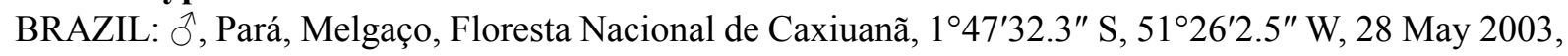
J.A.P. Barreiros and C.O. Araújo leg. (MPEG 0967).

\section{Paratypes}

BRAZIL: Pará, 1 q, Melgaço, Floresta Nacional de Caxiuanã, $1^{\circ} 47^{\prime} 32.3^{\prime \prime}$ S, 51 ${ }^{\circ} 26^{\prime} 2.5^{\prime \prime}$ W, 4 Oct. 2002, A. Chagas leg. (MPEG 0177); 1 §ै, same collection data as for preceding, 22 Oct. 2003, J.A. Barreiros leg. (IBSP 168432); 1 \%, same collection data as for preceding, Nov. 2002 (IBSP 168431).

\section{Additional material examined}

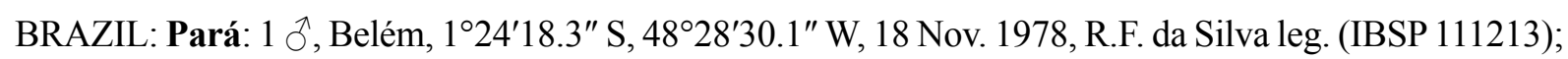
$1 \mathrm{O}^{\wedge}$, Santarém, $2^{\circ} 27^{\prime} 34.6^{\prime \prime} \mathrm{S}, 54^{\circ} 42^{\prime} 17.1^{\prime \prime} \mathrm{W}, 1991$, no data on collector (IBSP 107574); 1 \&, Floresta Nacional de Caxiuanã, 147'32.3" S, 5126'2.5" W, 26 May 2003, J.A.P Barreiros leg. (MPEG 0963); 1 q, same collection data as for preceding, 27 Mar. 2002 (MPEG 0194); 1 O, same collection data as

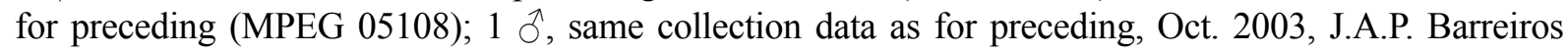

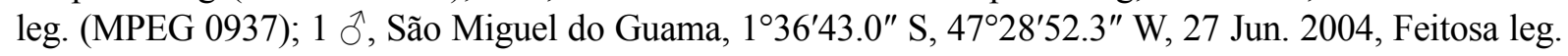
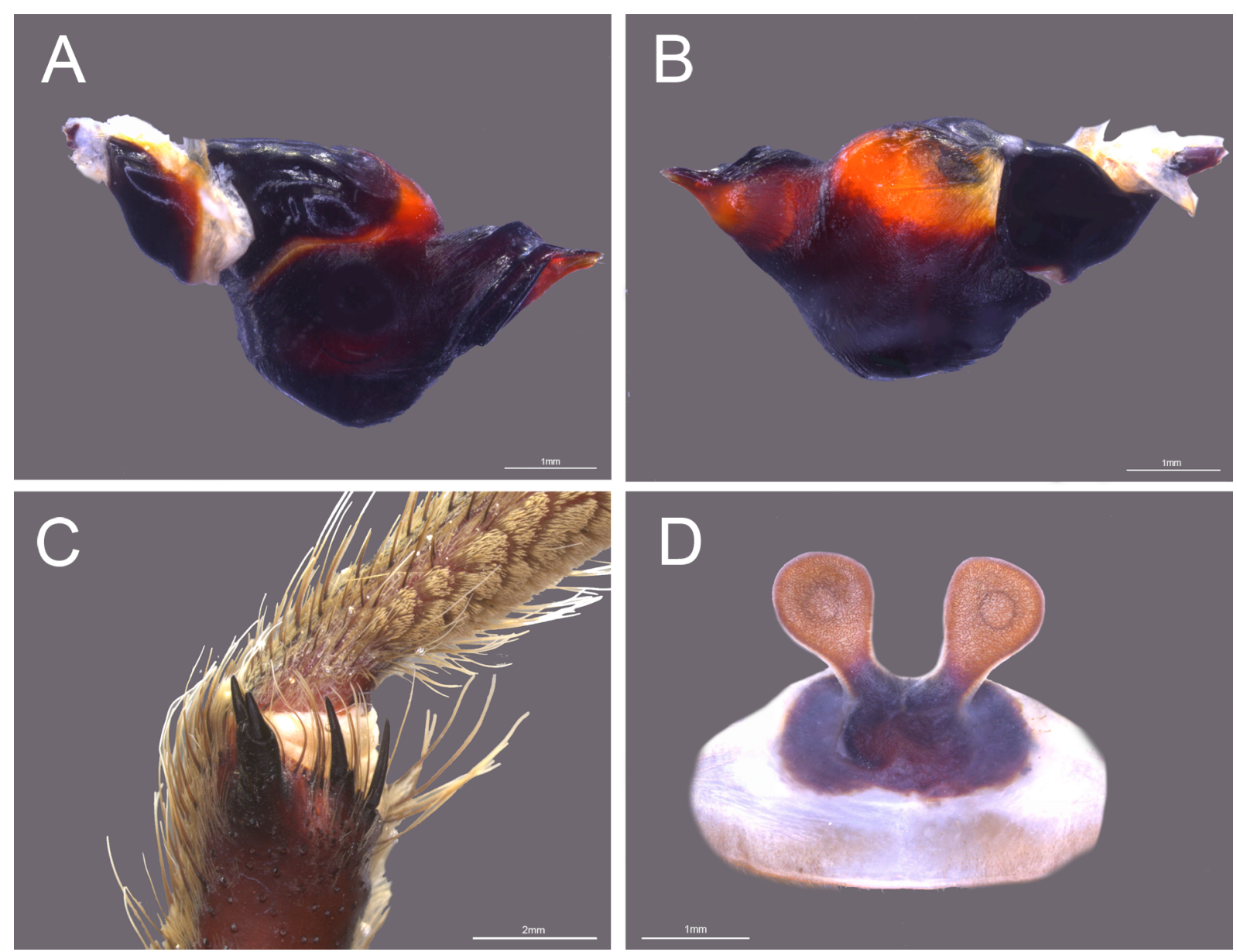

Fig. 27. Umbyquyra caxiuana gen. et sp. nov. A-C. Holotype, đ̂, Floresta Nacional de Caxiuanã, Pará, Brazil (MPEG 0967). A. Palp, prolateral. B. retrolateral. C. Tibial apophysis, retrolateral. D. Paratype,, , same locality, spermathecae, dorsal (MPEG 0177). 

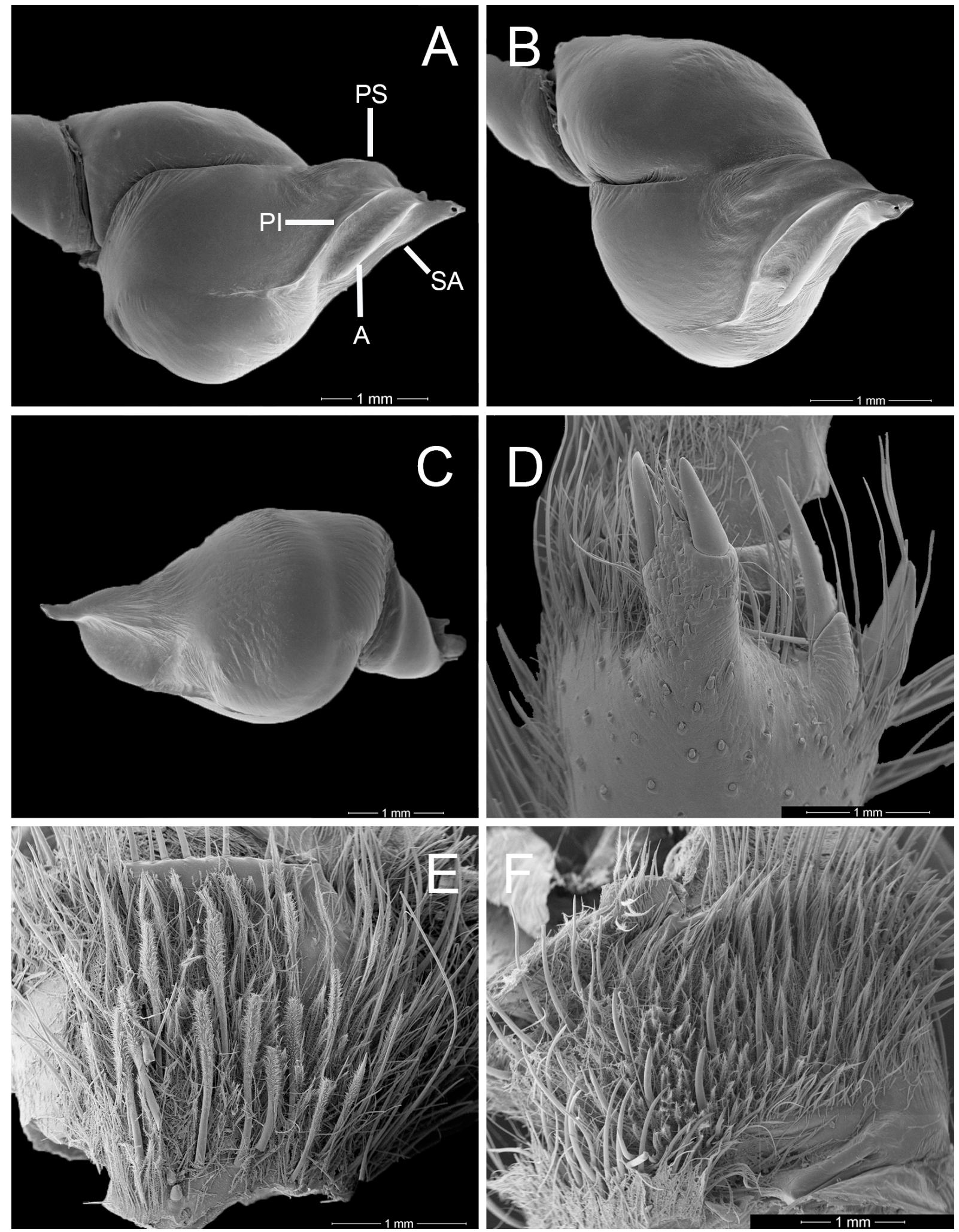

Fig. 28. Umbyquyra caxiuana gen. et sp. nov., holotype, $\widehat{\jmath}$, Floresta Nacional de Caxiuanã, Pará, Brazil (MPEG 0967). A. Palp, prolateral. B. Anterolateral. C. Retrolateral. D. Tibial apophysis, retrolateral. E. Stridulatory organ, trochanter. F. Same, trochanter I. Abbreviations: see Material and methods. 
(MPEG 5158); 1 ð, same collection data as for preceding, 23 Jul. 2003, J.A.P. Barreiros leg. (MPEG 0965); 1 , same collection data as for preceding (MPEG 0197); 1 त, same collection data as for preceding, 2003,

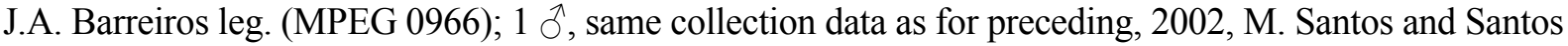
leg. (MPEG 09698); 1 §, Juruti, 02²8'22.1" S, 56²'12'29.4" W, 14 Feb. 2007, A. Lima and I. Pimenta leg. (MPEG 15450); 1 , same collection data as for preceding, 8 Jun. 2007, A. Lima and I. Pimenta leg. (MPEG 15451); 1 ○, same collection data as for preceding, 9 Jun. 2007, N.F. Lo-Man-Hung leg. (MPEG 15452); 1 ㅇ, same collection data as for preceding, 17 Nov. 2007, Souza leg. (MPEG 15453).

\section{Description}

Male (holotype MPEG 0967)

In ethanol: same color as $U$. belterra gen. et sp. nov. Total length: 47.0. Carapace: 20.8 long, 17.4 wide. Clypeus: 0.5. Eye tubercle 2.0 long, 2.8 wide. AME; 0.5 long, 0.5 wide; PME 0.4 long, 0.3 wide; ALE: 0.7 long, 0.5 wide; PLE 0.35 long, 0.4 wide. Basal segment of chelicerae with 10 teeth. Labium: 2.4 long, 2.5 wide, with 123 cuspules. Endites with 210-206 cuspules. Stridulatory bristles with around 20 bristles on palp trochanter, large and very long; stridulatory bristles on trochanter of leg I not so large, but surpassing 50 bristles (Fig. 28E-F). Palp: femur 9.7, patella 3.1, tibia 9.7, tarsus 3.6, total 26.1. Leg I femur 17.4, patella 6.2, tibia 14.7, metatarsus 14 , tarsus 9.4, total 61.7. II 16.5, 5.3, 15.1, 13.4, 8.5, 58.8. III 16.2, 7, 12.7, 15.7, 8.4, total 60. IV 16.2, 8.0, 16.7, 22, 9.4, 72.3. Spination: palp: femur d0-0-1app, tibia d0-0-1p-1p-1-2ap, p0-0-1-1-1-0ap. Legs I femur d0-0-1app, tibia v0-0-0-1r-1ap, p0-1-0-0ap, r0-1-0-1-0-0-0ap, metatarsus v0-1r-0-0ap. II femur, 0-0-0-1app, tibia v0-1-0-1apr-1app, p0-0-0-1-0ap, metatarsus v0-1-1-0-0app, p01-0-0ap. III femur d0-0-1apr, tibia v0-0-1-1-0-1app-1apr, p0-1-0-1-0ap, r0-1-0-1-0-1ap, metatarsus v01r-1p-0-1r-1p-0-1 app-1ap-2apr, p1-0-1-0-1-1 ap, r0-1-0-0ap. IV tibia v0-0-1r-1p-1p-1r-1p-1app-1ap-1apr, p0-1-0-1-0ap, r0-0-1-1ap, metatarsus d0-0-1r-0-0ap, v1p-1-1r-1-1p-1-1p-1r-1-1r-1r-1p-1r-1r-1apr-1app, p1-0-1-0-0-1ap, r0-1-0-1-2ap. Tibial apophysis of leg I: retrolateral branch elongated with two strong distal spines with three median setae on tubercle; prolateral branch projected with long distal and subdistal spines (Figs 27C, 28D). PLS basal, median and apical segments 2.1, 2.6, 3.5 long, respectively.

\section{Female (paratype, MPEG 0177)}

In ethanol: same color as male. Total length 52. Clypeus 0.4. AME 0.4 long, 0.4 wide; PME 0.2 long, 0.2 wide; ALE 0.2 long, 0.5 wide; PLE 0.2 long, 0.4 wide. Basal segment of chelicerae with 10 teeth. Labium 2 long, 2.5 wide, with 98 cuspules. Endites with 111-100 cuspules pointed. Eye tubercle 1.6 long, 2.8 wide. Stridulatory bristles as in male. Palp: femur 7.8, patella 4.6, tibia 4.1, tarsus 4.2, total 20.7. Leg I 11, 8.2, 6.3, 3.8, 3.5, 32.8. II 9.6, 6, 6.6, 7, 3.8, 33. III 8.1, 5.8, 7, 8.3, 4.1, 33.3. IV 10.5, 6.1, 8.6, 11.6, 4.2, 41. Spination: palp: tibia v1-0-1r-1r-1apr, p0-1-0ap. Legs I femur d0-0-1p-0ap, tibia v0-1-0-0-1ap, metatarsus v0-1-0-0-0-1ap-1apr. II tibia v0-1-0-1ap, p0-1-1-0ap, metatarsus v0-1-0-1app-1apr, p0-1-0ap, r0-0-2ap. III tibia v0-0-1 app-1 apr, p1-0-0ap, metatarsus d0-0-1r-0-0-1-0ap, v0-1-1p-1r-0-1r-1app, p1-1-1ap. IV tibia v-0-1p-1-1app-1apr, p0-1-1 ap, metatarsus d0-0-1-0ap, v1r-1r-1p-1r-2r-1p-1r-1r-1apr. PLS: basal, median and apical segments 4.0, 3.4, 3.8 long, respectively. Spermathecae with oval basal area (Fig. 27D).

\section{Distribution}

Brazil: state of Pará (Fig. 29).

\section{Species considered as nomen dubium}

Cyrtopholis meridionalis (Keyserling, 1891) nom. dub.

Cyrtosternum meridionalis Keyserling, 1891: 6.

Cyrtopholis meridionalis - Petrunkevitch 1911: 58. — Mello-Leitão 1926: 4.

Metriopelma meridionalis - Mello-Leitão 1923: 170. 
Keyserling (1891) described Cyrtosternum meridionalis based on a female from Taquara do Mundo Novo (nowadays Taquara), state of Rio Grande do Sul, Brazil, and did not mention where the holotype was deposited. Mello-Leitão (1923) translated the original text and transferred the species to Metriopelma Becker, 1878 with no further explanation. Later, Mello-Leitão (1926) transferred the species to Cyrtopholis. The original description (Keyserling 1891) does not allow an identification of the species, and the holotype supposedly in The Natural History Museum of London could not be located. Therefore, as the record in southern Brazil for this species is out of the distribution range of Umbyquyra gen. nov., Cyrtopholis meridionalis is herein considered as a nomen dubium.

\section{Synonymy}

Acanthoscurria gomesiana Mello-Leitão, 1923

Acanthoscurria gomesiana Mello-Leitão, 1923: 306, figs 51-55, 167.

Cyrtopholis zorodes Mello-Leitão, 1923: 143-144 (holotype 9 from São Paulo, São Paulo, Brazil, deposited in MNRJ 29, examined). syn. nov.

Phormictopus pheopygus Mello-Leitão, 1923: 273, figs 150-152.

Acanthoscurria violacea Mello-Leitão, 1923: 292, fig. 182.

Acanthoscurria pugnax Vellard, 1924: 142, pl. VIII, fig. 34a-d.

Acanthoscurria aurita Piza, 1939: 5, figs 2-3.

Acanthoscurria gomesiana - Schiapelli \& Gerschman 1964: 412, pl. III, fig. 9. - Gonzalez-Filho et al . 2012: 2, figs 1a-d, 2a-f, 5a-f.

Cyrtopholis zorodes - Bücherl et al. 1971: 124. - Silva-Moreira et al. 2010: 68.

Phormictopus pheopygus - Lucas 1983: 350 (syn.).

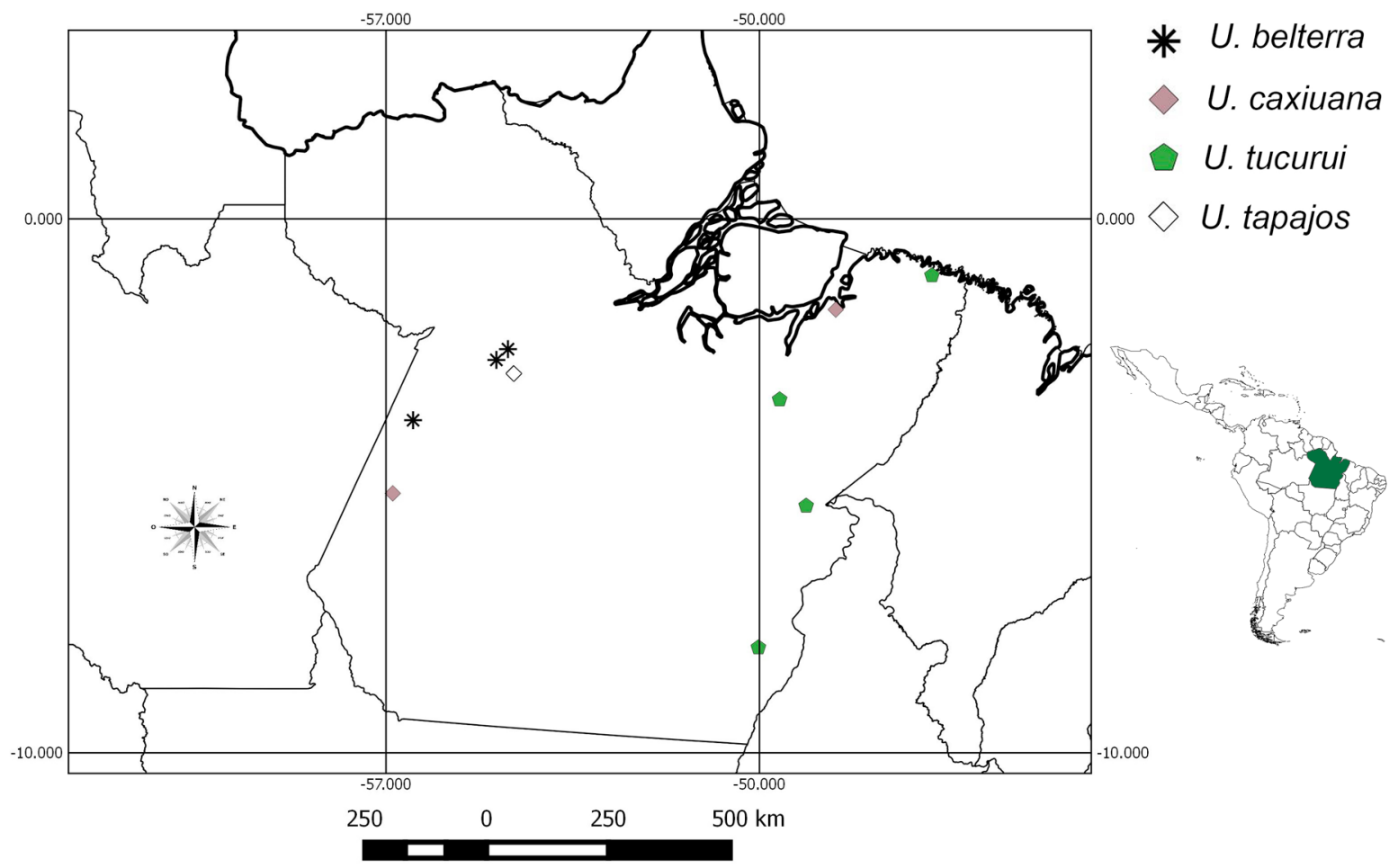

Fig. 29. Geographic distribution records of species of Umbyquyra gen. nov.: U. belterra gen. et sp. nov., $U$. caxiuana gen. et sp. nov., U. tucurui gen. et sp. nov. and U. tapajos gen. et sp. nov. 
Acanthoscurria violacea - Schiapelli \& Gerschman 1964: 415, pl. III, figs 19-21. — Gonzalez-Filho et al. 2012: 2 (syn.).

Acanthoscurria pugnax - Gonzalez-Filho et al. 2012: 2 (syn.).

Acanthoscurria aurita - Gonzalez-Filho et al. 2012: 2 (syn.).

\section{Remark}

The original description indicates MZSP as the type repository; however, no specimen of Cyrtopholis zorodes was located. Bücherl et al. (1971: 124) and Silva-Moreira et al. (2010: 68) agree that the specimen MNRJ 29, holotype + from São Paulo, in the state of São Paulo, Brazil is the true holotype. The holotype is in bad condition without SR and legs. Bücherl mentioned stridulatory bristles on the palp trochanter and first leg. The aspect of the SR and the type locality (São Paulo, state of São Paulo, Brazil) allow us to establish Cyrtopholis zorodes as a junior synonymy of Acanthoscurria gomesiana Mello-Leitão, 1923.

\section{Discussion}

Spiders of Theraphosinae in general present few morphological characters and several taxonomic problems (Raven 1990). Simon (1903) proposed the use of the stridulatory bristles, present in some Theraphosinae (Aviculariinae) to distinguish genera, according the localization on legs segments and pedipalps. He established four types of stridulatory bristles and the type IV, on the retrolateral face of the pedipalp and prolateral face of trochanter of leg I are nowadays recognized as distinctive characters in four genera: Acanthoscurria, Cyrtopholis, Nesipelma and Longilyra. According to this character, three species from South America were described: Cyrtopholis palmarum, C. schmidti and Acanthoscurria acuminata.
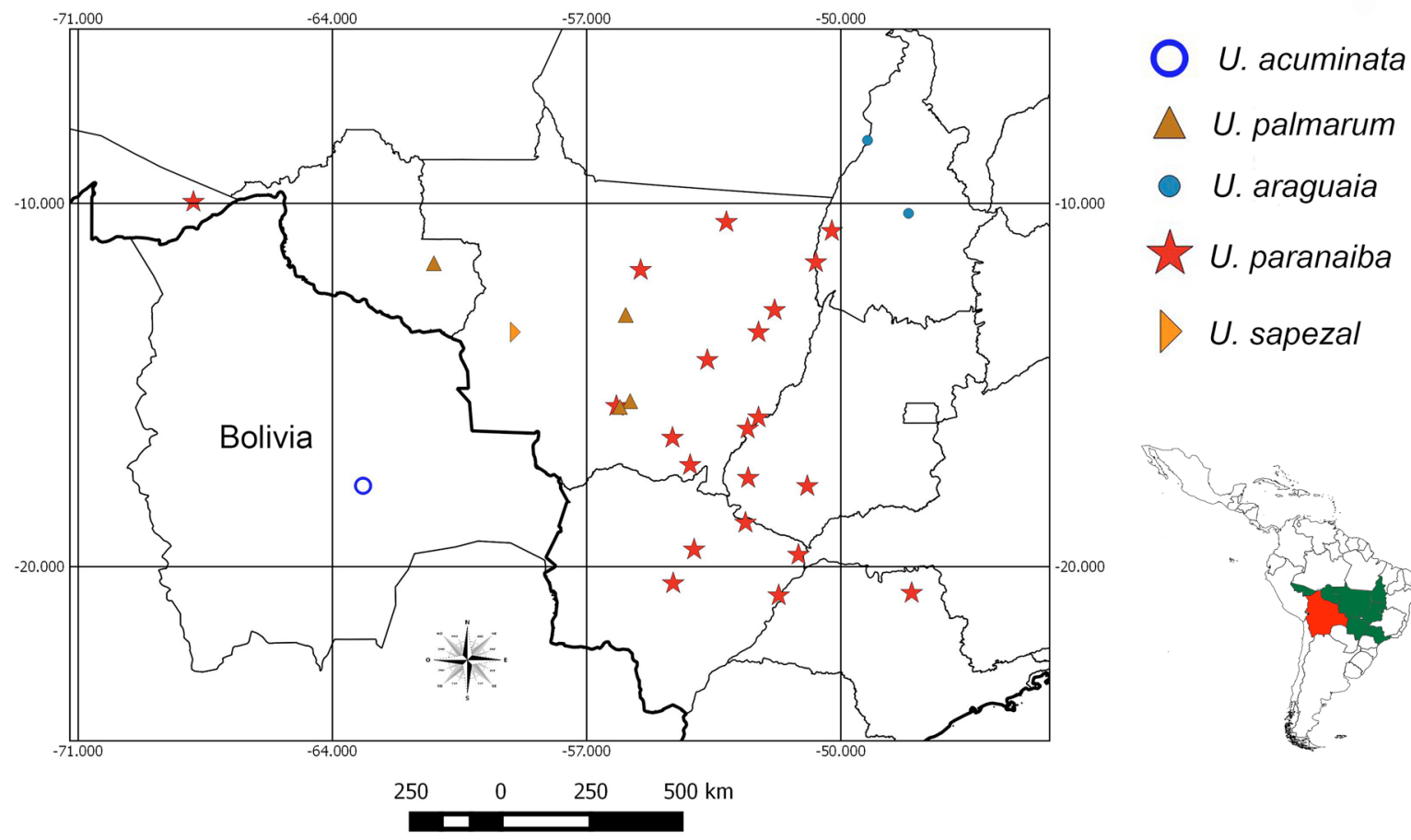

U. sapezal

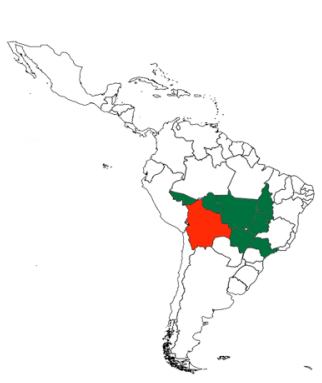

Fig. 30. Geographic distribution records of species of Umbyquyra gen. nov.: U. acuminata (Schmidt \& Tesmoingt in Schmidt, 2005) gen. et comb. nov., U. palmarum (Schiapelli \& Gerschman, 1945) gen. et comb. nov., U. araguaia gen. et sp. nov., U. paranaiba gen. et sp. nov. and U. sapezal gen. et sp. nov. 
Historically, the relationship between Cyrtopholis and Acanthoscurria has been referenced in the literature (Pérez-Miles et al. 1996; Bertani 2001), but Nesipelma and Longilyra have never been phylogenetically tested. Umbyquyra gen. nov. presents the same disposition of the stridulatory bristles, showing their relationship with these genera and this character could be a synapomorphy for this group within Theraphosinae.

It is noteworthy that the three species mentioned above, Umbyquyra palmarum gen. et comb. nov., $U$. schmidti gen. et comb. nov. and $U$. acuminata gen. et comb. nov. show a prominent projection of the cephalic region (Fig. 15E), another characteristic common in species of the Antillean genus Cyrtopholis (see Schmidt 2003: 268), were mistakenly included in Acanthoscurria. This character should be treated as plesiomorphic in Theraphosidae, as it also occurs in Ceratogyrus Pocock, 1897, an African Harpacterinae (De Wet \& Dippenaar-Schoeman 1991; Peters 2000) while Umbyquyra gen. nov. is included in Theraphosinae. Unfortunately, this character, highly variable among species, could hardly be used as diagnostic for these genera (Gallon, 2001). These structures range from almost absent to protruding projections on the back of the carapace (Fig. 5; De Wet \& Dippenaar-Schoeman 1991: fig. 4A-H) and their homology has not yet been studied among the various occurrences in Theraphosidae.

On the other hand, this study shows that in Umbyquyra gen. nov., as in general for Theraphosidae, the shape of the palp of the males and their structures are fundamental in the determination of the genera and their relations (Bücherl 1957; Pérez-Miles et al. 1996; Bertani 2000; Pérafan \& Pérez-Miles 2014; Ortiz \& Francke 2015). The pattern presented by Umbyquyra gen. nov. is very different from that of Cyrtopholis and Nesipelma, where the male palp bulb lacks an apophysis and has an elongated and tapered embolus (Figs 2A-B, 4C). In Longylira the males are still unknown, only females have been described. The palps of the males of Umbyquyra gen. nov. appear closer to Acanthoscurria since they

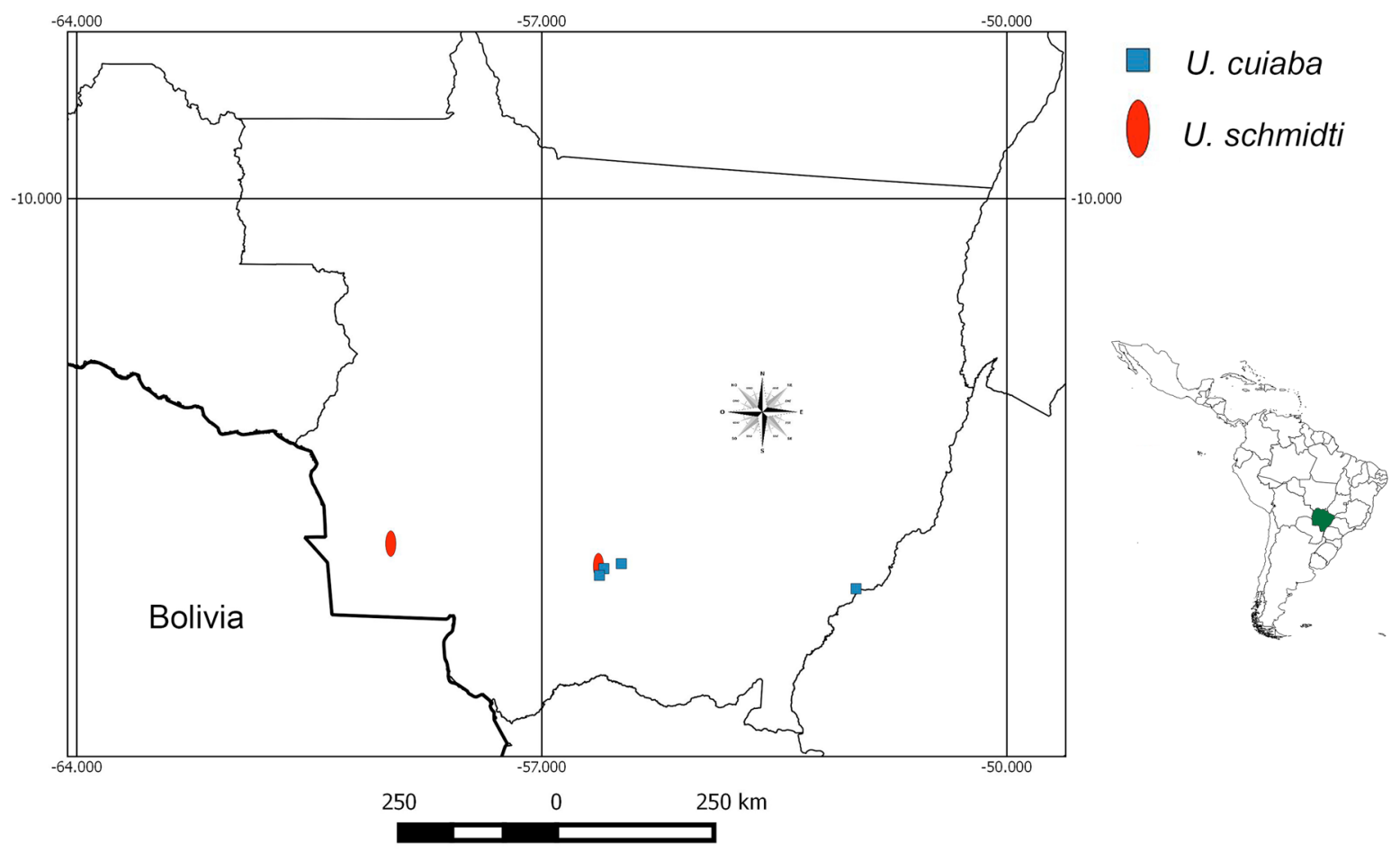

Fig. 31. Geographic distribution records of species of Umbyquyra gen. nov.: U. cuiaba gen. et sp. nov. and U. schmidti (Rudloff, 1996) gen. et comb. nov. 
present an apophysis, but are distinguished by the bulb of the palp with an acuminate and protruding bulb, and two tibial apophyses, whereas in the species of Acanthoscurria the apex of the embolus forms a spoon or furrow and presents only one tibial apophysis (Fig. 1C). The female genitalia in this group appears to be more conservative, but in Umbyquyra gen. nov. the seminal receptacles of the spermathecae have short ducts emerging from a large, sclerotic base (Fig. 7D).

Although we found some putative relationships, a phylogeny of this group is still necessary, but it would be equally important to review of Cyrtopholis, a genus with a wide diversity in the Antilles, and to increase the knowledge of the monotypic Nesipelma and Longilyra, the latter known only by females.

\section{Acknowledgments}

Our special thanks to Rodolfo R. da Silva, who in the 80 's collected and brought us numerous specimens of Umbyquyra paranaiba. Also, special thanks to Volker von Wirth for studying and taking photos of the holotype of Acanthoscurria acuminata. To Davi F. Candiani, who identified the Theraphosinae of MPEG and brought the specimens to our laboratory for study. To R.P. Indicatti, who collected and sent us the first living female of U. cuiaba. To Beatriz Maurício, for her help with SEM images. To C. Perafán and P. Jäger, who examined the holotype of Nesipelma insulare and sent us photos. In addition, we wish to thank the curators for the loan of specimens as well as V. Passanha for the help in the formatting of the plates, R. Gabriel and C. Grismado for sending us photos, and Gabriel de los Santos and Alexandre Sanchez Ruiz for sending us specimens of Cyhrtopholis from the Domican Republican.

\section{References}

Bertani R. 2000. Male palpal bulbs and homologous features in Theraphosinae (Araneae, Theraphosidae). Journal of Arachnology 28: 29-42. https://doi.org/10.1636/0161-8202(2000)028\%5B0029:MPBAHF\%5D2.0.CO;2

Bertani R. 2001. Revision, cladistic analysis, and zoogeography of Vitalius, Nhandu, and Proshapalopus; with notes on other theraphosine genera (Araneae, Theraphosidae). Arquivos de Zoologia 36: 265-356.

Bücherl W. 1957. Sôbre a importância dos bulbos copuladores e das apófises tibiais dos machos na sistemática das aranhas caranguejeiras (Orthognatha). Anais da Academia Brasileira de Ciências 29: $377-416$.

Bücherl W., Timotheo da Costa A. \& Lucas S. 1971. Revisão de alguns tipos de aranhas caranguejeiras (Orthognatha) estabelecidos por Cândido de Mello-Leitão e depositados no Museu Nacional do Rio. Memórias do Instituto Butantan 35: 117-138.

De Wet J.I. \& Dippenaar-Schoeman A.S. 1991. A revision of the genus Ceratogyrus Pocock (Araneae: Theraphosidae). Koedoe 34: 39-68. https://doi.org/10.4102/koedoe.v34i2.422

Gabriel R. 2014. A new genus and species of theraphosid spider from El Salvador (Araneae: Theraphosidae). British Tarantula Society Journal 29: 146-153.

Gallon R.C. 2001. Revision of the Ceratogyrus spp. formerly included In Coelogenium (Araneae: Theraphosidae, Harpactirinae). Mygalomorph 2: 1-20.

Gerschman de P. B.S. \& Schiapelli R.D. 1973. La subfamilia Ischnocolinae (Araneae: Theraphosidae). Revista del Museo Argentino de Ciencias Naturales Bernardino Rivadavia 4: 43-77.

Gonzalez-Filho H.M.O., Lucas S.M., Paula F.S., Indicatti R.P. \& Brescovit A.D. 2012. On the taxonomy of Acanthoscurria Ausserer from Southeastern Brazil with data on the natural history of A. gomesiana Mello-Leitão (Araneae, Mygalomorphae, Theraphosidae). International Journal of Zoology 721793: 1-11. https://doi.org/10.1155/2012/721793 
GARGIULO F. de F. et al., Umbyquyra gen. nov., new genus of Neotropical Theraphosidae

Guadanucci J.P.L. 2014. Theraphosidae phylogeny: relationships of the 'Ischnocolinae' genera (Araneae, Mygalomorphae). Zoologica Scripta 43: 508-518. https://doi.org/10.1111/zsc.12065

Keyserling E. 1891. Die Spinnen Amerikas. Dritter Band. Brasilianische Spinnen. Verlag von Bauer \& Raspe, Nürnberg.

Lucas S.M. 1983. Sobre a distribuição geográfica dos gêneros da subfamília Theraphosinae Thorell, 1870 no Brasil (Araneae, Theraphosidae). Memórias do Instituto Butantan 46: 339-352.

Mello-Leitão C.F. 1923. Theraphosoideas do Brasil. Revista do Museu Paulista 13: 1-438.

Mello-Leitão C.F. 1926. Algumas Theraphosoideas novas no Brasil. Revista do Museu Paulista 14: 307-324.

Ortiz D. \& Francke O.F. 2015. Two new species of Bonnetina tarantulas (Theraphosidae, Theraphosinae) from Mexico: contributions to morphological nomenclature and molecular characterization of types. Journal of Natural History 49: 685-707. https://doi.org/10.1080/00222933.2014.924770

Perafán C. \& Pérez-Miles F. 2014. Three new species of Melloleitaoina Gerschman \& Schiapelli, 1960 (Araneae, Mygalomorphae, Theraphosidae) from northern Argentina. ZooKeys 404: 117-129.

https://doi.org/10.3897/zookeys.404.6243

Perafán C., Cifuentes Y.Y. \& Estrada-Gomez S. 2015. Aguapanela, a new tarantula genus from the Colombian Andes (Araneae, Theraphosidae). Zootaxa 4033: 529-542.

https://doi.org/10.11646/zootaxa.4033.4.4

Pérez-Miles F., Lucas S.M., Silva Jr. P.I. da \& Bertani, R. 1996. Systematic revision and cladistic analysis of Theraphosinae (Araneae: Theraphosidae). Mygalomorph 1: 33-68.

Peters H.-J. 2000. Tarantulas of the World: kleiner Atlas der Vogelspinnen - Band 1. Published by the author.

Petrunkevitch A. 1911. A synonymic index catalogue of spiders of North, Central and South America with all adjacents islands, Greenland, Bermudas, West Indies, Terra del Fuego, Galapagos, etc. Bulletin of American Museum of Natural History 29: 1-791. https://doi.org/10.5962/bhl.title.23819

Petrunkevitch A. 1925. Arachnida from Panama. Transactions of the Connecticut Academy of Arts and Sciences 27: 51-248.

Piza S. de T. Jr. 1939. Novas aranhas do Brasil. Revista de Agricultura, São Paulo 14: 1- 2.

Raven R. 1985. The spider infraorder Mygalomorph (Araneae). Cladistic and systematics. Bulletin of the American Museum of Natural History 182: 1-180.

Raven R. 1990. A new species of Linothele from Colombia (Araneae, Mygalomorphae, Dipluridae). Journal of Arachnology 18: 79-86.

Rudloff J.-P. 1996. Cyrtopholis schmidti sp. n., eine neue Cyrtopholis-Art aus Brasilien (Araneida: Theraphosidae: Theraphosinae). Arachnologisches Magazin 4: 2-8.

Schiapelli R.D. \& Gerschman B.S. 1945. Parte descriptiva. In: Vellard J., Schiapelli R.D. \& Gerschman de Pikelin B.S. (eds.) Arañas sudamericanas colleccionadas por el Doctor J. Vellard. I. Theraphosidae nuevas o poco conocidas. Acta Zoologica Lilloana 3: 165-213.

Schiapelli R.D. \& Gerschman B.S. 1964. El género Acanthoscurria (Araneae, Theraphosidae) en la Argentina. Physis 24: 391-417.

Schmidt G. 1993. Vogelspinnen: Vorkommen, Lebensweise, Haltung und Zucht, mit Bestimmungsschlüsseln für alle Gattungen. Fourth edition. Landbuch Verlag, Hannover. 
Schmidt G. 1997. Bestimmungsschlüssel für die Gattungen der Unterfamilie Theraphosinae (Araneae: Theraphosidae). Arachnologisches Magazin 3: 1-27.

Schmidt G. 2000. Das Männchen von Acanthoscurria acuminata Schmidt \& Tesmoingt, 2000 (Araneae: Theraphosidae: Theraphosinae). Arachnologisches Magazin 8: 1-4.

Schmidt G. 2003. Die Vogelspinnen: eine weltweite Übersicht. Neue Brehm-Bücherei 641. WestarpWissenschaften. Hohenwarsleben.

Schmidt G. 2005. Neupublikation einiger von mir in "Arachnol. Mag." und "Tarantulas of the World" zwischen 2000 und 2005 veröffentlichten Arbeiten. Tarantulas of the World 110: 3-49.

Schmidt G. \& Tesmoingt M. 2000. Eine weitere Acanthoscurria-Art aus Brasilien und Bolivien (Araneae: Theraphosidae: Theraphosinae). Arachnologisches Magazin 8: 1-6.

Silva-Moreira T., Baptista R.L.C., Kury A.B., Giupponi A.P.L., Buckup E.H. \& Brescovit A.D. 2010. Annotated check list of Arachnida type specimens deposited in the Museu Nacional, Rio de Janeiro. II-Araneae. Zootaxa 2588: 1-91.

Simon E. 1903. Histoire naturelle des araignées: 669-1080. Paris 2.

Vellard J. 1924. Études de Zoologie. Archivos do Instituto Vital Brazil 2: 121-170.

Vellard J. 1945. Observaciones biológicas. In: Vellard J., Schiapelli R.D. \& Gerschman de Pikelin B.S. (eds) Arañas sudamericanas colleccionadas por el Doctor J. Vellard. I. Theraphosidae nuevas o poco conocidas. Acta Zoológica Lilloana 3: 195-213.

World Spider Catalog 2016. World Spider Catalog, version 17.0. Natural History Museum Bern. Available from http://wsc.nmbe.ch [accessed 5 Apr. 2017].

Manuscript received: 16 August 2017

Manuscript accepted: 11 June 2018

Published on: 23 August 2018

Topic editor: Rudy Jocqué

Desk editor: Pepe Fernández

Printed versions of all papers are also deposited in the libraries of the institutes that are members of the EJT consortium: Muséum national d'Histoire naturelle, Paris, France; Botanic Garden Meise, Belgium; Royal Museum for Central Africa, Tervuren, Belgium; Natural History Museum, London, United Kingdom; Royal Belgian Institute of Natural Sciences, Brussels, Belgium; Natural History Museum of Denmark, Copenhagen, Denmark; Naturalis Biodiversity Center, Leiden, the Netherlands; Museo Nacional de Ciencias Naturales-CSIC, Madrid, Spain; Real Jardín Botánico de Madrid CSIC, Spain; Zoological Research Museum Alexander Koenig, Bonn, Germany. 